quatrième série-tome $51 \quad$ fascicule $3 \quad$ mai-juin 2018

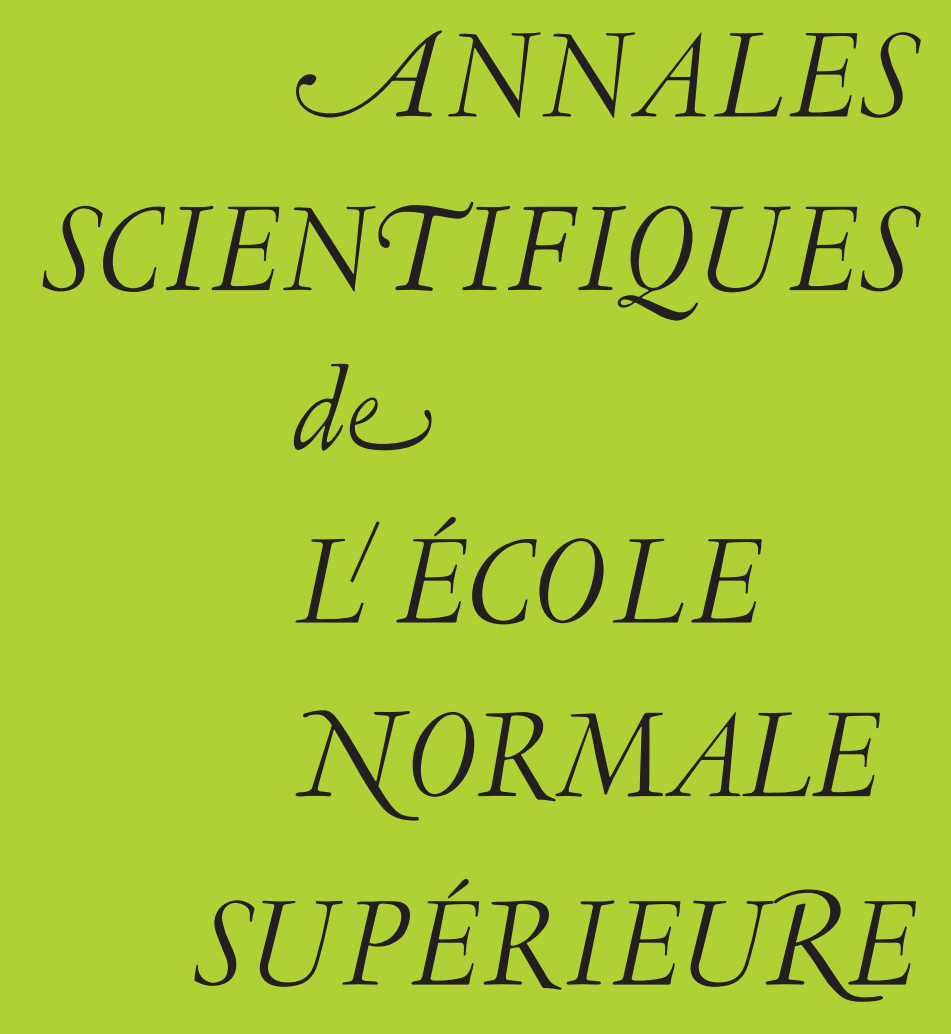

Fabrizio ANDREATTA \& Adrian IOVITA \& Vincent PILLONI Le balo spectral 


\title{
Annales Scientifiques de l'École Normale Supérieure
}

Publiées avec le concours du Centre National de la Recherche Scientifique

\author{
Responsable du comité de rédaction / Editor-in-chief
}

Patrick BERNARD

Publication fondée en 1864 par Louis Pasteur

Continuée de 1872 à 1882 par H. Sainte-Claire Deville
Comité de rédaction au $1^{\mathrm{er}}$ mars 2018
P. BERNARD
A. Neves
S. BOUCKSOM
J. SzeFtel
R. CERF
S. VŨ NGỌC
G. Chenevier
A. Wienhard
Y. DE CORNULIER
G. Williamson
E. Kowalski

\section{Rédaction / Editor}

Annales Scientifiques de l'École Normale Supérieure,

45, rue d'Ulm, 75230 Paris Cedex 05, France.

Tél. : (33) 1443220 88. Fax : (33) 144322080.

annales@ens.fr

\section{Édition et abonnements / Publication and subscriptions}

Société Mathématique de France

Case 916 - Luminy

13288 Marseille Cedex 09

Tél. : (33) 0491267464

Fax : (33) 0491411751

email : smf@smf .univ-mrs.fr

\section{Tarifs}

Abonnement électronique : 420 euros.

Abonnement avec supplément papier :

Europe : $540 €$. Hors Europe : $595 €(\$ 863)$. Vente au numéro : $77 €$.

C) 2018 Société Mathématique de France, Paris

En application de la loi du $1^{\text {er }}$ juillet 1992, il est interdit de reproduire, même partiellement, la présente publication sans l'autorisation de l'éditeur ou du Centre français d'exploitation du droit de copie (20, rue des Grands-Augustins, 75006 Paris).

All rights reserved. No part of this publication may be translated, reproduced, stored in a retrieval system or transmitted in any form or by any other means, electronic, mechanical, photocopying, recording or otherwise, without prior permission of the publisher. 
Ann. Scient. Éc. Norm. Sup.

$4^{\text {e }}$ série, t. 51, 2018, p. 603 à 655

\title{
LE HALO SPECTRAL
}

\author{
PAR FABrizIo ANDREATTA, AdriAn IOVITA \\ ET VINCENT PILLONI
}

RÉSUMÉ. - Nous construisons un espace de formes surconvergentes en caractéristique $p$, un opérateur compact sur cet espace et nous montrons que la série caractéristique de cet opérateur est la réduction modulo $p$ de la série universelle de Coleman. Nous démontrons que les formes surconvergentes en caractéristique $p$ de pente finie se déforment vers la caractéristique 0 .

Abstract. - We construct a space of overconvergent modular forms of characteristic $p$, a compact operator on this space and we show that the characteristic series of this operator is the reduction modulo $p$ of Coleman's universal series. We prove that finite slope overconvergent modular forms in positive characteristic can be deformed to characteristic 0 .

\section{Introduction}

\subsection{Présentation des résultats}

Soit $p$ un nombre premier et $N$ un entier premier à $p$. Soit $X$ la courbe modulaire sur $\mathbb{Z}_{p}$ de niveau $N$. Soit $\bar{X}$ sa réduction modulo $p, \bar{X}_{\text {ord }}$ le lieu ordinaire et $\mathfrak{X}_{\text {ord }}$ le schéma formel $p$-adique lieu ordinaire. Soit $\bar{x}$ un point géométrique de $\bar{X}_{\text {ord }}$ et $\rho: \Pi_{1}\left(\bar{X}_{\text {ord }}, \bar{x}\right) \rightarrow \mathbb{Z}_{p}^{\times}$la représentation donnée par la tour d'Igusa qui paramètre les trivialisations de la partie multiplicative du module de Tate du schéma semi-abélien universel. Pour tout caractère $\kappa: \mathbb{Z}_{p}^{\times} \rightarrow \mathbb{C}_{p}^{\times}$, l'équivalence de Katz [21] associe à $\kappa \circ \rho$ un $\phi$-module étale $\omega^{\kappa}$ sur $\mathfrak{X}_{\text {ord }}$. Ses sections globales sont l'espace $M_{\kappa}^{p \text {-ad }}$ des formes modulaires $p$-adiques de poids $\kappa$. L'opérateur de Dwork associé est $U_{p}=p^{-1} \operatorname{Tr}_{\phi}$. Dans [21] si $\kappa$ est algébrique, puis dans [10] (complété par [12], [7] et revisité dans [3] et [25]), il est démontré que le $\phi$-module $\omega^{\kappa}$ surconverge. Ses sections surconvergentes sont l'espace $M_{\kappa}^{\dagger}$ des formes modulaires surconvergentes de poids $\kappa$.

L'opérateur $U_{p}$ est compact et on peut définir, d'après [27], la série caractéristique $\mathscr{P}_{\kappa}(X):=\operatorname{det}\left(1-X U_{p} \mid M_{\kappa}^{\dagger}\right)$ qui est une fonction entière de la variable $X$. 
Notons $\Lambda=\mathbb{Z}_{p}\left[\left[\mathbb{Z}_{p}^{\times}\right]\right]$l'algèbre d'Iwasawa. Coleman montre de plus l'existence d'une série caractéristique universelle $\mathscr{P}(X) \in \Lambda[[X]]$ qui interpole les différentes séries caractéristiques $\mathscr{P}_{\kappa}(X)$. Notons $\mathscr{W}^{\text {rig }}=(\operatorname{Spf} \Lambda)^{\text {rig }}$ l'espace rigide des poids, qui est une union finie $\amalg \mathscr{W}_{\chi}^{\mathrm{rig}}$ de boules ouvertes de rayon 1 paramétrées par les caractères $\chi:(\mathbb{Z} / q \mathbb{Z})^{\times} \rightarrow \mathbb{C}_{p}^{\times}$ (où $q=4$ si $p=2$ et $q=p$ sinon). Coleman et Mazur définissent la variété spectrale $\mathscr{Z}^{\text {rig }}=V(\mathscr{P}) \subset \mathscr{Q}^{\text {rig }} \times \mathbb{A}^{1}$. Ses points sont des couples $\left(\kappa, \alpha^{-1}\right)$ où $\kappa$ est un poids et $\alpha$ une valeur propre non nulle de $U_{p}$ agissant sur $M_{\kappa}^{\dagger}$. Ils définissent également la courbe de Hecke $\mathcal{E}^{\text {rig }} \rightarrow \mathscr{Z}^{\text {rig }}$ dont les points sont des triplets $\left(\kappa, \alpha^{-1}, \lambda\right)$ où $\left(\kappa, \alpha^{-1}\right) \in Z^{\text {rig }}$ et $\lambda$ est un système de valeurs propres de Hecke agissant sur l'espace propre $\left(M_{\kappa}^{\dagger}\right)^{U_{p}=\alpha}$.

Bien que la construction de la variété de Hecke soit de nature rigide analytique, il est surprenant que la série caractéristique $\mathscr{P}(X)$ soit à coefficients entiers. Ceci suggère la possibilité d'une théorie entière des formes surconvergentes. Ce travail a pour but d'établir une telle théorie. Notre point de départ est une conjecture de Coleman sur la réduction modulo $p$ de la série caractéristique. Fixons un isomorphisme $\Lambda \simeq \mathbb{Z}_{p}\left[(\mathbb{Z} / q \mathbb{Z})^{\times}\right][[T]]$, en envoyant $\exp (q)$ sur $1+T$. Pour tout caractère $\chi$ de $(\mathbb{Z} / q \mathbb{Z})^{\times}$, notons $\mathcal{P}_{\chi}(X) \in \mathbb{Z}_{p}[[T]][[X]]$ la $\chi$-partie de $\mathscr{P}(X)$ et $\overline{\mathcal{P}_{\chi}}(X) \in \mathbb{F}_{p}[[T]][[X]]$ sa réduction modulo $p$. Notons aussi $\bar{\kappa}_{\chi}: \mathbb{Z}_{p}^{\times} \rightarrow \mathbb{F}_{p}[[T]]^{\times}$le caractère obtenu par réduction du caractère universel et projection sur la $\chi$-partie (on remarquera que si $p=2, \bar{\kappa}_{\chi}$ est indépendant de $\chi$ ). Dans [11], Coleman observe que c'est une série entière de la variable $X$ à coefficients dans l'anneau $\mathbb{F}_{p}[[T]]$ équipé de la topologie $T$-adique et il conjecture le résultat suivant :

Conjecture 1 ([11]). - Pour chaque caractère $\chi d u$ groupe $(\mathbb{Z} / q \mathbb{Z})^{\times}$, on possède un $\mathbb{F}_{p}((T))$-espace de formes surconvergentes $M_{\bar{\kappa}_{\chi}}^{\dagger}$ de poids $\bar{\kappa}_{\chi}$ et un opérateur compact $U_{p}$ dont la série caractéristique est $\overline{\mathcal{P}_{\chi}}(X)$.

Pour tout caractère $\bar{\kappa}_{\chi}$ comme au-dessus, l'équivalence de Katz associe à $\bar{\kappa}_{\chi} \circ \rho$ : $\Pi_{1}\left(\bar{X}_{\text {ord }}, \bar{x}\right) \rightarrow \mathbb{F}_{p}[[T]]^{\times}$un $\phi$-module étale $\omega^{\bar{\kappa}_{\chi}}$ sur le schéma formel $T$-adique $\mathfrak{X}_{\text {ord, }\{\infty\}}:=$ $\bar{X}_{\text {ord }} \times_{\text {Spec } \mathbb{F}_{p}} \operatorname{Spf} \mathbb{F}_{p}[[T]]$. Notons $\mathscr{C}_{\{\infty\}}$ l'espace rigide sur $\mathbb{F}_{p}((T))$ associé au schéma formel $\bar{X} \times_{\text {Spec } \mathbb{F}_{p}} \operatorname{Spf} \mathbb{F}_{p}[[T]]$ et $\mathscr{C}_{\text {ord, }\{\infty\}} \subset \mathcal{C}_{\{\infty\}}$ l'ouvert ordinaire. On peut alors se demander si le $\phi$-module étale $\omega^{\bar{\kappa}_{\chi}}$ surconverge sur un voisinage de $\mathscr{C}_{\text {ord, }\{\infty\}}$ dans $\mathscr{C}_{\mathfrak{C}}$.

THÉORÈme 1.1. - Le $\phi$-module $\omega^{\bar{\kappa}_{\chi}}$ est surconvergent.

Nous définissons alors $M_{\bar{\kappa}_{\chi}}^{\dagger}$ comme l'espace des sections surconvergentes de $\omega^{\bar{\kappa}_{\chi}}$. On possède sur cet espace un opérateur compact $U_{p}$ associé à $\phi$. On peut définir sa série caractéristique, et on veut vérifier qu'elle vaut $\overline{\mathscr{P}_{\chi}}(X)$. Il nous faut à présent relier cet espace aux espaces de formes surconvergentes de caractéristique 0 .

Commençons par «compactifier» l'espace des poids. L'idée est d'ajouter à $W^{\text {rig }}$ les caractères $\bar{\kappa}_{\chi}$. Cet espace des poids compactifié possèdera donc des points de caractéristique 0 et $p$, on sort du cadre classique de la géométrie rigide, et nous utilisons à présent la théorie des espaces adiques de Huber. Définissons donc $\mathscr{W}=\operatorname{Spa}(\Lambda, \Lambda)^{\mathrm{an}}$, c'est l'ouvert des points analytiques de Spa $(\Lambda, \Lambda)$. Comme ensemble, $\mathscr{W}=\mathscr{W}^{\text {rig }} \bigcup\left\{\bar{\kappa}_{\chi}, \chi: \mathbb{Z} / q \mathbb{Z}^{\times} \rightarrow \mathbb{C}_{p}^{\times}\right\}$(dorénavant $\mathscr{W}^{\text {rig }}$ désigne l'espace adique associé à $\left.\mathscr{W}^{\text {rig }}\right)$. Tout voisinage ouvert de $\left\{\bar{\kappa}_{\chi}\right\}$ dans $\mathscr{W}$ contient une couronne de rayon extérieur 1 dans la composante connexe $\mathscr{W}_{\chi}^{\text {rig }}$ de $\mathscr{W}^{\text {rig }}($ resp. dans chaque composante connexe de $\mathscr{W}^{\text {rig }}$ si $p=2$ ). 
On possède un faisceau stuctural $\left(\mathscr{O}_{\omega}, \mathscr{O}_{\omega}^{+}\right)$et sur $\mathscr{O}_{\omega}^{+}$la topologie est $(p, T)$-adique. Plus précisément, sur tout ouvert quasi-compact de $\mho^{\text {rig }}$, la topologie $(p, T)$-adique coïncide avec la topologie $p$-adique. Inversement, sur tout ouvert quasi-compact qui évite les centres $T=0$ des disques ouverts $\mathscr{W}_{\chi}^{\text {rig }}$ la topologie $(p, T)$-adique coïncide avec la topologie $T$-adique. On assiste donc, à mesure qu'on se rapproche du bord de $\mathscr{W}$, à un glissement de la topologie $p$-adique vers la topologie $T$-adique.

Considérons la courbe modulaire relative $\mathcal{M}_{[0, \infty]}=X \times_{\text {Spec } \mathbb{Z}_{p}} W \rightarrow W$ et son ouvert ordinaire $\mathcal{M}_{\text {ord, }[0, \infty]}$. La construction de Katz appliquée au caractère universel $\mathbb{Z}_{p}^{\times} \rightarrow \Lambda^{\times}$

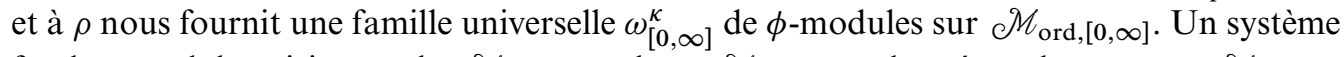
fondamental de voisinages de $\mathcal{M}_{\text {ord, }[0, \infty]}$ dans $\mathcal{M}_{[0, \infty]}$ est donné par les ouverts $M_{r,[0, \infty]}$ d'équation :

$$
\left|\tilde{\mathrm{Ha}}^{p^{r+1}}\right| \geq \sup \{|T|,|p|\}
$$

où Ha désigne un relèvement arbitraire local de l'invariant de Hasse. Si on regarde ces voisinages pour la topologie $p$-adique sur l'ouvert $\mathscr{W}^{\text {rig }}$, alors ils se rétrécissent vers le lieu ordinaire à l'infini. Dans [3] et [25], nous avons montré la surconvergence de $\omega_{[0, \infty]}^{\kappa}$ au-dessus de $\mathscr{Q}^{\text {rig }}$ et dans le théorème 1.1 nous avons montré la surconvergence à l'infini. Le théorème suivant unifie ces deux résultats :

THÉORÈME 1.2. - La famille universelle de $\phi$-modules $\omega_{[0, \infty]}^{\kappa}$ sur $\mathcal{M}_{\mathrm{ord},[0, \infty]}$ surconverge sur $M_{r,[0, \infty]}$ pour $r \geq 3$ (resp. $r \geq 5$ si $p=2$ ).

La série caractéristique associée à cette famille de $\phi$-modules est à coefficients dans $\Lambda=\mathrm{H}^{0}\left(\mathscr{W}, \mathscr{O}_{\omega}\right)$. Par fonctorialité, on vérifie que c'est bien la série caractéristique de Coleman. Nous sommes donc en mesure d'établir la conjecture 1 :

Corollaire 1.1. - La série caractéristique de $U_{p}$ sur $M_{\bar{\kappa}_{\chi}}^{\dagger}$ vaut $\overline{\mathscr{P}_{\chi}}$.

Soit $\mathscr{Z}=V(\mathscr{P}) \subset \mathscr{Q} \times \mathbb{A}^{1}$ la variété spectrale adique. Sa fibre au-dessus de $\mathscr{W}^{\text {rig }}$ est $\mathscr{Z}^{\text {rig }}$. Sa fibre à l'infini est l'ensemble des points $\left(\bar{\kappa}_{\chi}, \alpha^{-1}\right)$ où $\alpha$ est une valeur propre non nulle de $U_{p}$ agissant sur $M_{\bar{\kappa}_{\chi}}^{\dagger}$. On possède sur $\mathscr{Z}$ un faisceau cohérent universel $M^{\dagger}$ d'espaces caractéristiques de formes surconvergentes et une courbe de Hecke adique $\mathcal{E} \rightarrow \mathscr{Z}$ où $\mathscr{O}_{\mathcal{E}}$ est le sous-faisceau de $\operatorname{End}_{\mathcal{Z}}\left(M^{\dagger}\right)$ engendré par les opérateurs de Hecke. Le théorème suivant généralise les résultats du chapitre 7 de [12]. Il montre l'existence de familles de pente finie pour la topologie $T$-adique au voisinage de l'infini.

THÉORÈME 1.3. - Le morphisme $\mathscr{Z} \rightarrow$ W est localement fini et plat sur $\mathscr{Z}$. Le morphisme $\mathcal{E} \rightarrow$ W est localement fini et sans torsion.

Corollaire 1.2. - Soit $f \in M_{\bar{\kappa}_{\chi}}^{\dagger}$ une forme propre surconvergente de pente finie. Soit $x_{f} \in \mathcal{E}$ le point associé. Il existe un voisinage $\mathscr{W}_{\bar{\kappa}_{\chi}}$ de $\bar{\kappa}_{\chi}$ dans $W^{U}$ et un voisinage $\mathcal{E}_{x_{f}}$ de $x_{f}$ dans $\mathcal{E}$ tel que le morphisme associé $\mathcal{E}_{x_{f}} \rightarrow \mathscr{W}_{\bar{\kappa}_{x}}$ soit fini et sans torsion.

De plus, pour tout entier $k \geq 2$, on peut trouver une suite $\left(f_{n}\right)_{n \in \mathbb{N}}$ de formes propres classiques de pente finie et de poids $k$, de point associé $x_{f_{n}}$ sur $\mathcal{E}_{x_{f}}$, tel que $x_{f_{n}} \rightarrow x_{f}$ quand $n \rightarrow \infty$. 
Les formes $f_{n}$ du corollaire ont un niveau $\Gamma_{1}\left(p^{m_{n}}\right)$ en $p$ et $m_{n}$ tend vers l'infini. Même si leur poids classique est $k$, leur poids $p$-adique est un caractère localement algébrique de $\mathbb{Z}_{p}^{\times}$ de la forme $z \mapsto z^{k} \chi_{n}(z)$ avec $\chi_{n}(z)$ un caractère d'ordre fini et de conducteur $p^{m_{n}}$.

\subsection{Questions et perspectives}

1.2.1. Polygone de Newton au bord de l'espace des poids. - Buzzard et Kilford [8] ont étudié la variété spectrale en niveau 1 pour $p=2$. Ils ont démontré que pour les poids $\kappa$ vérifiant $v_{2}(\kappa(5)-1)<3$ et $\kappa(1)=-1$, les pentes de l'opérateur $U_{p}$ sur l'espace des formes de poids $\kappa$ sont $0, t, 2 t, 3 t, \ldots$ où $t=v_{2}(\kappa(5)-1)$ et $v_{2}$ est la valuation 2-adique normalisée $\operatorname{par} v_{2}(2)=1$. La généralisation suivante de ce résultat est conjecturée :

Conjecture 2 ([22]). - Soit $\left(n_{i}, m_{i}\right)$ les points de rupture du polygone de Newton de $\overline{\mathscr{P}}_{\chi}$. Il existe $r<1$ tel que pour tout $\kappa$ sur la couronne d'équation $r<|T|<1$ de $\mathscr{W}_{\chi}$, les points de rupture du polygone de Newton de $\mathscr{P}_{\kappa}$ sont $\left(n_{i}, v_{p}(\kappa(1+q)-1) m_{i}\right)$.

Pour les formes surconvergentes quaternioniques, cette conjecture est démontrée dans la publication [22]. Signalons également que dans [4], il est démontré que la conjecture entraîne que les pentes du polygone de Newton de $\overline{\mathscr{P}}_{\chi}$ forment une union finie de progressions arithmétiques. Ce résultat est obtenu indépendamment dans [22].

QUESTION 1.1. - Existe-t-il un opérateur géométrique sur $M_{\bar{\kappa}_{\chi}}^{\dagger}$ qui explique cette progression arithmétique?

1.2.2. Dimension supérieure. - Les résultats de cet article devraient pouvoir se généraliser sans trop de problème à des variétés de Shimura PEL plus générales. Remarquons que le bord de l'espace des poids compactifié sera toujours un fermé de codimension 1, et donc de dimension positive dès que le rang du groupe est au moins 2. A notre connaissance, il n'y a pas de conjecture sur les pentes de Newton au bord en dimension supérieure.

1.2.3. Théorie de Hodge. - Soit $f \in M_{\bar{\kappa}_{\chi}}^{\dagger}$ une forme surconvergente de pente finie, propre pour l'algèbre de Hecke. On peut alors lui associer une représentation semi-simple continue :

$$
\rho_{f}: G_{\mathbb{Q}} \rightarrow \mathrm{GL}_{2}\left(\overline{\mathbb{F}}_{p}[[t]]\right)
$$

Ici $t$ est une variable, en fait $t$ est une uniformisante d'une extension finie de $\overline{\mathbb{F}}_{p}((T))$. Cette représentation est impaire et vérifie la compatibilité locale-globale hors de $p$ et des places ramifiées. De plus det $\rho_{f}=\left(\bar{\kappa}_{\chi}^{-1} \circ \chi_{\text {cycl }}\right) \cdot \omega$ où $\chi_{\text {cycl }}$ désigne le caractère cyclotomique et $\omega$ sa réduction modulo $p$. Se pose la question de décrire la représentation $\left.\rho_{f}\right|_{G_{\mathbb{Q} p}}$. Si $f$ de pente nulle vérifie $U_{p} f=\alpha . f$, alors d'après la théorie de Hida ([31], thm. 2),

$$
\left.\rho_{f}\right|_{G_{\mathbb{Q} p}} \simeq\left(\begin{array}{cc}
n r(\alpha) & \star \\
0 & n r\left(\alpha^{-1}\right)\left(\bar{\kappa}_{\chi}^{-1} \circ \chi_{\mathrm{cycl}}\right) . \omega
\end{array}\right)
$$

où $n r(\alpha)$ est le caractère non ramifié qui applique le Frobenius géométrique sur $\alpha$.

Dans le cas de pente finie, on peut espérer que les choses se passent comme en caractéristique 0 . Soit $\mathbb{D}$ la boule unité ouverte de la variable $Z$ sur le corps non-archimédien $\overline{\mathbb{F}}_{p}((t))$. On définit des actions de $\phi$ et $\Gamma=\mathbb{Z}_{p}^{\times}$par les formules habituelles $\phi(Z)=Z^{p}$ et $\gamma(Z)=(1+Z)^{\gamma}-1$. L'anneau de Robba $\mathscr{R}$ est (dans ce contexte) l'anneau des séries $f=\sum_{n=-\infty}^{\infty} a_{n} Z^{n}$ à coefficients dans $\overline{\mathbb{F}}_{p}((t))$ qui converge sur une couronne non vide 
$0<v(Z)<r(f)$. Un $(\phi, \Gamma)$-module est un $\mathscr{R}$-module libre de rang fini muni d'actions semi-linéaires de $\phi$ et $\Gamma$ qui commutent, et telles que le linéarisé de $\phi$ soit un isomorphisme. En utilisant les méthodes de [5], on démontre :

ThÉORÈme 1.4. - A toute représentation continue $\rho: G_{\mathbb{Q}_{p}} \rightarrow \operatorname{GL}_{n}\left(\overline{\mathbb{F}}_{p}((t))\right)$ on peut associer un $(\phi, \Gamma)$-module $\mathscr{D}(\rho)$.

On devrait pouvoir (suivant [17]) construire une filtration de Harder-Narishiman sur la catégorie des $(\phi, \Gamma)$-modules et démontrer qu'on a une équivalence entre représentations de $G_{\mathbb{Q}_{p}}$ et $(\phi, \Gamma)$-modules semi-stables de pente 0 .

QUESTION 1.2. - Soit $\rho_{f}$ la représentation associée à une forme modulaire de pente finie. Le $(\phi, \Gamma)$-module $\mathscr{D}\left(\rho_{f}\right)$ est-il triangulin?

Plus précisément, on devrait posséder une suite exacte $0 \rightarrow L \rightarrow \mathscr{D}\left(\rho_{f}\right) \rightarrow L^{\prime} \rightarrow 0$ où $L$ est le $(\phi, \Gamma)$-module de rang 1 avec action triviale de $\Gamma$.

QUESTION 1.3. - Peut-on caractériser quand une représentation semi-simple continue impaire $\rho: G_{\mathbb{Q}} \rightarrow \mathrm{GL}_{2}\left(\overline{\mathbb{F}}_{p}((T))\right)$ est la représentation associée à une forme surconvergente de pente finie?

On espère revenir bientôt sur ces questions.

\subsection{Description de l'article}

Dans la section 2, nous définissons la compactification de l'espace des poids W dans la catégorie des espaces adiques. Nous expliquons que la topologie est $p$-adique au centre $T=0$ et $T$-adique au bord $p=0$. Nous étudions également les propriétés d'analyticité du caractère universel. Plus on est proche du centre $T=0$ de l'espace des poids, plus le caractère est analytique. En revanche, au bord $p=0$, le caractère n'est pas localement analytique.

Dans la section 3, nous appliquons la théorie du sous-groupe canonique aux courbes modulaires. Nous construisons, par éclatement, des modèles formels de voisinages stricts $\mathrm{du}$ lieu ordinaire. Nous définissons aussi des revêtements «tour d'Igusa partielle» de ces voisinages stricts. Ce sont là des constructions classiques dans la théorie.

Dans la section 4, nous définissons les espaces de formes surconvergentes en caractéristique $p$. La situation en caractéristique $p$ est très spéciale. D'une part, on possède des sous-groupes canoniques de tous ordres donnés par les noyaux des itérés du Frobenius. Un tel énoncé n'est vrai que sur le lieu ordinaire en caractéristique 0. D'autre part, le caractère universel de $\mathbb{Z}_{p}^{\times}$n'est pas localement analytique, alors qu'il l'est en caractéristique 0 . Nous construisons une tour d'Igusa surconvergente d'ordre infini, et nous montrons que la construction de Katz en caractéristique $p$ garde un sens dans le contexte surconvergent. Nous vérifions ensuite qu'elle fournit des bons objets. Cela repose au final sur une étude fine de la ramification de la tour d'Igusa aux points supersinguliers.

Dans la section 5, nous reprenons notre construction de la famille universelle de faisceaux sur l'espace rigide des poids usuel. Cette construction repose sur l'analyticité locale du caractère universel et sur l'existence de sous-groupes canoniques d'ordre fini sur des voisinages stricts du lieu ordinaire. Nous améliorons nos résultats antérieurs car nous construisons aussi 
des modèles entiers canoniques inversibles pour nos faisceaux de formes surconvergentes. Faisons une remarque importante qui justifie le changement de la topologie $p$-adique vers la topologie $T$-adique : plus le caractère est proche du bord de l'espace des poids, moins il est localement analytique et plus on a besoin d'avoir un sous-groupe canonique d'ordre grand pour faire la construction. Traditionnellement, on utilisait la topologie $p$-adique pour décrire l'espace des poids et plus on voulait travailler avec des caractères proches du bord, plus on devait rapetisser les voisinages du lieu ordinaire. En réalité, si on utilise la topologie $T$-adique au voisinage du bord de l'espace des poids (ce qui est plus naturel), on peut construire la famille de faisceaux sur l'espace rigide sur un voisinage strict de rayon constant !

Dans la section 6, nous recollons les constructions sur l'espace rigide et en caractéristique $p$. Nous n'avons pas réussi à faire cela simplement pour deux raisons. En caractéristique $p$ nous avons utilisé le sous-groupe canonique d'ordre infini qui ne surconverge pas en caractéristique 0 . En caractéristique 0 nous avons utilisé l'analyticité locale du caractère universelle qui est fausse en caractéristique $p$. Pour recoller les constructions, nous avons utilisé un revêtement pro-fini perfectoïde d'un voisinage strict du lieu ordinaire : «la tour anti-canonique ». Au-dessus de ce revêtement on a (tautologiquement) la surconvergence de la tour d'Igusa. Cela permet, comme si on était sur le lieu ordinaire, de donner une définition uniforme (et donc indépendante de la caractéristique) du faisceau des formes modulaires surconvergentes «perfectisées ». On démontre ensuite, au moyen de traces de Tate, que le faisceau perfectisé descend et réalise le recollement des faisceaux construits dans les sections 4 et 5. Cela nous permet finalement de construire une courbe de Hecke adique et de vérifier la conjecture de Coleman.

L'article contient deux appendices. Le premier est consacré à la théorie du sous-groupe canonique que nous reformulons au niveau de généralité dont nous avons besoin : sans supposer que la topologie est $p$-adique et sans extraire de racines de $p$. Le second appendice est consacré à la théorie spectrale de Coleman que nous étendons aux espaces adiques analytiques.

\subsection{Remerciements}

Nous remercions P. Scholze pour une discussion très intéressante et sa suggestion d'utiliser les espaces adiques pour compactifier l'espace des poids. Nous remercions également J. Bergdall, B. Stroh et L. Xiao pour des échanges stimulants. Durant la préparation de ce travail, nous avons séjourné à l'ENS Lyon, à l'université de Milan et au MSRI. Nous remercions le rapporteur pour ses nombreux commentaires. Nous remercions le programme ANR14-CE25-0002 pour son financement. Ce travail est dédié à la mémoire de Robert Coleman, qui en est l'inspirateur.

\section{L'espace des poids}

\subsection{Définition}

Soit $\Lambda=\mathbb{Z}_{p}\left[\left[\mathbb{Z}_{p}^{\times}\right]\right]$l'algèbre d'Iwasawa. Nous allons lui associer plusieurs espaces adiques ([20], [18], [19]).

4 e SÉRIE - TOME $51-2018$ - No 3 
2.1.1. Points analytiques. - Posons $q=4$ si $p=2$ ou $q=p$ si $p \neq 2$. On a $\mathbb{Z}_{p}^{\times} \simeq$ $\mathbb{Z} / q \mathbb{Z}^{\times} \times \exp (q)^{\mathbb{Z}_{p}}$. On obtient alors un isomorphisme $\Lambda \simeq \mathbb{Z}_{p}\left[\mathbb{Z} / q \mathbb{Z}^{\times}\right][[T]]$ en posant $1+T=\exp (q)$ et $\Lambda$ est complet pour la topologie $(p, T)$-adique.

Soit $\mathfrak{W}=\operatorname{Spa}(\Lambda, \Lambda)$. C'est l'espace des classes d'équivalences de valuations continues $\operatorname{sur} \Lambda$, il est équipé d'un faisceau en algèbres topologiques $\mathscr{O}_{\mathfrak{W}}$ et de plus, pour chaque point $x \in \mathfrak{W}$ on dispose d'une valuation $v_{x}$ sur la fibre $\mathscr{O}_{\mathfrak{W}, x}$.

Rappelons que si $\left(A, A^{+}\right)$est une algèbre affinoïde, et si $x \in \operatorname{Spa}\left(A, A^{+}\right)$, on appelle support de $x$ l'idéal premier de $A$ des éléments $a \in A$ tels que $|a|_{x}=0$. Suivant [19], sect. 3, on dit qu'un point est analytique si son support n'est pas ouvert.

Lemme 2.1. - Les points non analytiques de $\mathfrak{W}$ sont en bijection avec $\operatorname{Spf} \Lambda$.

Démonstration. $-\mathrm{Si} x$ est non analytique, alors $|\cdot|_{x}$ se factorise en une valuation sur $\Lambda /(p, T)$. Son support est donc un idéal maximal de $\Lambda$. Soit $\mathfrak{m}$ un idéal maximal de $\Lambda$ (qui correspond au choix d'un caractère à valeur dans $\mathbb{F}_{p} p^{\times}$de $\mathbb{Z} / q \mathbb{Z}^{\times}$). On peut lui associer la valuation définie par $v_{\mathfrak{m}}(a)=0$ si $a \in \mathfrak{m}, v_{\mathfrak{m}}(a)=1$ sinon, qui nous donne la bijection cherchée.

DéfInition 2.1. - Soit $ひ$ l'ouvert constitué des points analytiques de $\mathfrak{W}$.

2.1.2. Description de $\mathscr{W}$. - Comme l'idéal de définition de $\Lambda$ est engendré par $p$ et $T$, pour décrire $W$ on va comparer, pour tout point $|.| \in \mathscr{W}$, les éléments $|p|$ et $|T|$. Pour tout rationnel $\frac{r}{s} \in \mathbb{Q}_{>0}$, on note :

$$
\begin{aligned}
& \mathscr{W}_{\leq \frac{r}{s}}=\left\{x \in \mathscr{W}_{,}\left|T^{\mathrm{r}}\right|_{x} \leq\left|p^{\mathrm{s}}\right|_{x} \neq 0\right\}, \\
& \mathscr{W}_{\geq \frac{r}{s}}=\left\{x \in \mathscr{W},\left|p^{\mathrm{s}}\right|_{x} \leq\left|T^{\mathrm{r}}\right|_{x} \neq 0\right\} .
\end{aligned}
$$

On pose également :

$$
\begin{aligned}
\mathscr{W}_{\geq 0} & =\mathscr{W}_{\leq \infty}=\mathscr{W}, \\
\mathscr{W}_{\leq 0} & =\left\{x \in \mathscr{W},|T|_{x}=0\right\}, \\
\mathscr{W}_{\geq \infty} & =\left\{x \in \mathscr{W},|p|_{x}=0\right\} .
\end{aligned}
$$

Si $I=[a, b] \subset[0, \infty]$ est un intervalle avec $a, b \in \mathbb{Q}_{\geq 0} \cup\{\infty\}$, on pose $\mathscr{W}_{I}=\mathscr{W}_{\leq b} \cap \mathscr{W}_{\geq a}$. Si $I \neq\{0\}$ et $I \neq\{\infty\}$, $W_{I}$ est un ouvert rationnel. On laisse le soin au lecteur de formuler la définition de $\mathscr{W}_{I}$ pour un intervalle général $I \subset[0, \infty]$.

Soit $x \in \mathscr{W}$ une valuation de rang 1 . On peut définir la valuation $p$-adique de $x$ par

$$
v_{p}(x)=\frac{\log _{p}\left(|T|_{x}\right)}{\log _{p}\left(|p|_{x}\right)} \in[0, \infty] .
$$

On vérifie alors facilement que $x \in \mathscr{W}_{I} \Leftrightarrow v_{p}(x)^{-1} \in I$.

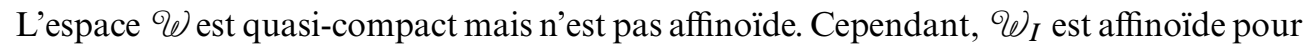
tout intervalle fermé à coordonnées rationnelles $I \subsetneq[0, \infty]$. Par exemple, $\mathscr{W}=\mathscr{W}_{[0,1]} \cup W_{[1, \infty]}$ avec par définition :

$$
\mathscr{W}_{[0,1]}=\operatorname{Spa}\left(\Lambda\left\langle\frac{T}{p}\right\rangle\left[\frac{1}{p}\right], \Lambda\left\langle\frac{T}{p}\right\rangle\right) \text { et } \mathscr{W}_{[1, \infty]}=\operatorname{Spa}\left(\Lambda\left\langle\frac{p}{T}\right\rangle\left[\frac{1}{T}\right], \Lambda\left\langle\frac{p}{T}\right\rangle\right) .
$$

La topologie sur $\Lambda$ est la topologie $(p, T)$-adique. Soit $t \in \mathbb{Q}_{>0}$. La topologie est $p$-adique dans $\mathscr{O}_{\mathscr{W}_{[0, t]}^{+}}^{+}$et $p$ est une unité topologiquement nilpotente dans $\mathscr{O}_{\mathscr{\omega}_{[0, t]}}$. La topologie est 
$T$-adique sur $\mathscr{O}_{\omega_{[t,+\infty]}^{+}}$et $T$ est une unité topologiquement nilpotente dans $\mathscr{O}_{\left.\omega_{[t,+\infty}\right]}$. Sur un intervalle rationnel $[a, b] \subset] 0,+\infty\left[\right.$, les topologies $p$ et $T$-adiques coïncident sur $\mathscr{O}_{\omega_{[a, b]}^{+}}^{+}$ et $p, T$ sont des unités dans $\mathscr{O}_{\omega_{[a, b]}}$.

2.1.3. Le bord. $-\mathrm{Si} t \in\left[0,+\infty\left[\mathscr{W}_{[0, t]}\right.\right.$ est une réunion finie de boules de centre 0 et de rayon $p^{-\frac{1}{t}}$. Posons $\mathscr{W}^{\text {rig }}=\mathscr{W}_{[0,+\infty[}$. C'est l'espace adique associé à la fibre générique de $\operatorname{Spf} \Lambda$ au sens de Berthelot ([6], sect. 0), et c'est une réunion finie de boules ouvertes de rayon 1. C'est l'espace des poids qui est traditionnellement considéré dans la théorie des familles analytiques de formes surconvergentes. Le bord de l'espace des poids est par

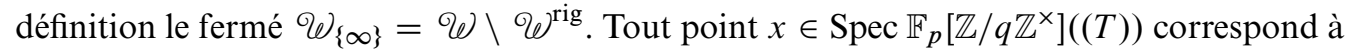
un morphisme

$$
\mathbb{F}_{p}\left[\mathbb{Z} / q \mathbb{Z}^{\times}\right]((T)) \stackrel{x}{\rightarrow} \mathbb{F}_{p}((T)) .
$$

On peut associer à $x$ une valuation $v_{x}$ sur $\Lambda$, obtenue en composant le morphisme de réduction $\Lambda \rightarrow \mathbb{F}_{p}\left[\mathbb{Z} / q \mathbb{Z}^{\times}\right]((T))$, le morphisme $x$, et la valuation $T$-adique sur $\mathbb{F}_{p}((T))$.

Lemme 2.2. - Le bord $\mathscr{W}_{\{\infty\}}$ est l'ensemble des valuations $v_{x}$ pour $x \in \operatorname{Spec} \mathbb{F}_{p}\left[\mathbb{Z} / q \mathbb{Z}^{\times}\right]((T))$.

Démonstration. - Par définition, $\mathscr{W}_{\{\infty\}}$ est l'ensemble des points analytiques ayant $p$ dans leur support. Il en résulte aussitôt que c'est l'ensemble fini des valuations $v_{x}$.

On spécifie alors la structure (non réduite si $p=2$ ) d'espace adique sur $\mathscr{W}_{\{\infty\}}$ en posant :

$$
\mathscr{W}_{\{\infty\}}=\mathscr{W}_{\operatorname{Spa}\left(\mathbb{Z}_{p}, \mathbb{Z}_{p}\right)} \operatorname{Spa}\left(\mathbb{F}_{p}, \mathbb{F}_{p}\right)=\operatorname{Spa}\left(\mathbb{F}_{p}\left[\mathbb{Z} / q \mathbb{Z}^{\times}\right]((T)), \mathbb{F}_{p}\left[\mathbb{Z} / q \mathbb{Z}^{\times}\right][[T]]\right) .
$$

2.1.4. Composante libre et composante finie. - On note $\omega^{0}=\operatorname{Spa}\left(\mathbb{Z}_{p}[[T]], \mathbb{Z}_{p}[[T]]\right)^{\text {an }}$. C'est

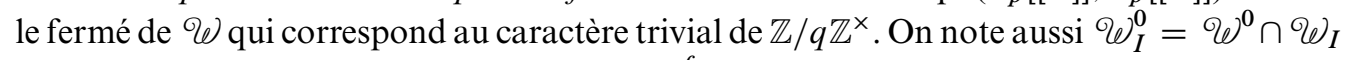
pour tout intervalle $I \subset[0,+\infty]$. On note $\mathscr{\omega}^{f}=\operatorname{Spa}\left(\mathbb{Z}_{p}\left[\mathbb{Z} / q \mathbb{Z}^{\times}\right], \mathbb{Z}_{p}\left[\mathbb{Z} / q \mathbb{Z}^{\times}\right]\right)$l'espace des caractères de $\mathbb{Z} / q \mathbb{Z}^{\times}$. On a la décomposition

$$
\mathscr{W}=\mathscr{U}^{0} \times{ }_{\mathrm{Spa}\left(\mathbb{Z}_{p}, \mathbb{Z}_{p}\right)} \mathscr{W}^{f} .
$$

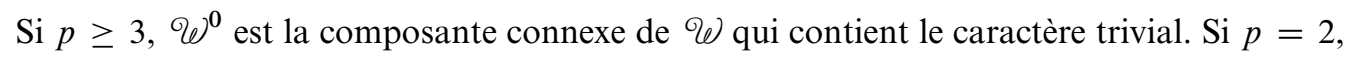
W est connexe!

\subsection{Analyticité du caractère universel}

On note $\mathbb{G}_{a}^{+}$le préfaisceau en anneaux sur la catégorie des espaces adiques défini $\operatorname{par} \mathbb{G}_{a}^{+}(X)=\mathrm{H}^{0}\left(X, \mathscr{O}_{\mathrm{X}}^{+}\right)$. On note $\mathbb{G}_{m}^{+}$le sous-préfaisceau en groupes de $\mathbb{G}_{a}^{+}$des éléments inversibles pour la multiplication.

On dispose du caractère universel $\kappa^{\mathrm{un}}: \mathbb{Z}_{p}^{\times} \rightarrow \Lambda^{\times}$. Ce caractère fournit un accouplement de préfaisceaux (où $\mathbb{Z}_{p}^{\times}$est le préfaisceau constant de valeur $\mathbb{Z}_{p}^{\times}$) :

$$
\mho_{0} \times \mathbb{Z}_{p}^{\times} \rightarrow \mathbb{G}_{m}^{+} \text {. }
$$

Si on restreint $\kappa^{\text {un }}$ à certains ouverts de l'espace des poids, alors le caractère devient localement analytique, c'est-à-dire qu'il se prolonge en un caractère du groupe $\mathbb{Z}_{p}^{\times}\left(1+p^{n} \mathbb{G}_{a}^{+}\right) \subset \mathbb{G}_{m}^{+}$.

Lemme 2.3. - Pour tout $n \geq 1$, on a $\kappa^{\mathrm{un}}\left(1+q p^{n-1} \mathbb{Z}_{p}\right)-1 \subset\left(T^{p^{n-1}}, T^{p^{n-2}} p, \ldots, p^{n-1} T\right) \Lambda$. 
Démonstration. - Rappelons que la valuation $p$-adique de $C_{p^{n}}^{k}$ vaut $n-v_{p}(k)$. On a donc $\kappa^{\mathrm{un}}\left(\exp \left(q p^{n-1}\right)\right)=(1+T)^{p^{n-1}}=\sum_{k=0}^{p^{n-1}} C_{p^{n-1}}^{k} T^{k}=1 \bmod \left(T^{p^{n-1}}, T^{p^{n-2}} p, \ldots, p^{n-1} T\right)$.

Proposition 2.1. - Pour tout $n \geq 1$, le caractère universel nous fournit un accouplement:

$$
\mathscr{W}_{\left[0, p^{\mathrm{n}} q^{-1}\right]} \times \mathbb{Z}_{p}^{\times}\left(1+q p^{n-1} \mathbb{G}_{a}^{+}\right) \rightarrow \mathbb{G}_{m}^{+}
$$

qui se restreint en un accouplement :

$$
\mathscr{W}_{\left[0, p^{\mathrm{n}} q^{-1}\right]} \times\left(1+q p^{n-1} \mathbb{G}_{a}^{+}\right) \rightarrow 1+q \mathbb{G}_{a}^{+} .
$$

Démonstration. - Soit $\left(R, R^{+}\right)$une algèbre affinoïde complète et soit le morphisme $g: \operatorname{Spa}\left(R, R^{+}\right) \rightarrow \mathscr{W}_{\left[0, p^{\mathrm{n}} q^{-1}\right]}$ donné par un morphisme $g:\left(\mathscr{O}_{W_{\left[0, p^{\mathrm{n}} q^{-1}\right]}}, \mathscr{O}_{\mho_{\left[0, p^{\mathrm{n}} q^{-1}\right]}^{+}}\right) \rightarrow$ $\left(R, R^{+}\right)$. Il s'agit alors de construire un caractère $\kappa_{g}: \mathbb{Z}_{p}^{\times}\left(1+q p^{n-1} R^{+}\right) \rightarrow\left(R^{+}\right)^{\times}$. La restriction du caractère à $\mathbb{Z}_{p}^{\times}$est donné par la composition

$$
\mathbb{Z}_{p}^{\times} \rightarrow \Lambda^{\times} \rightarrow \mathscr{O}_{\mathscr{W}_{\left[0, p^{\mathrm{n}} q^{-1}\right]}^{+\times}}^{+} \rightarrow R^{+\times}
$$

On a des isomorphismes inverses donnés par l'exponentielle et le logarithme $q p^{n-1} R^{+} \simeq$ $1+q p^{n-1} R^{+}$. D'autre part, $\kappa^{\text {un }}(\exp (q))=1+T$ et $\kappa^{\text {un }}\left(\exp \left(q p^{n-1}\right)\right)=(1+T)^{p^{n-1}}$. Or, $(1+T)^{p^{n-1}}=1+q h(T)$ où $h(T) \in \mathscr{O}_{\omega_{\left[0, p^{\mathrm{n}} q-1\right.}^{+}}^{+}[T]$ d'après le lemme précédent. Posons donc, pour tout $r \in R^{+}, \kappa_{f}\left(\exp \left(q p^{n-1} r\right)\right)=f(\exp (r \log (1+q h(T))))$. Le fait que le caractère envoie $1+p^{n-1} q \mathbb{G}_{a}^{+}$dans $1+q \mathbb{G}_{a}^{+}$est évident sur la formule.

\section{Courbes modulaires et tour d'Igusa}

\subsection{Courbes modulaires formelles et voisinages du lieu ordinaires}

Soit $X \rightarrow$ Spec $\mathbb{Z}_{p}$ la courbe modulaire compactifiée de niveau $N \geq 3$ premier à $p$ ([13], IV, thm. 2.5). Soit $\mathfrak{X} \rightarrow \operatorname{Spf} \mathbb{Z}_{p}$ sa complétion formelle $p$-adique. Soit $E$ le schéma semi-abélien universel sur $\mathfrak{X}$, et $\omega_{E}$ le faisceau co-normal en la section neutre de $E$. Soit $\mathrm{Ha}:=\operatorname{Ha}(E) \in \mathrm{H}^{0}\left(\mathfrak{X}, \omega_{E}^{p-1} \otimes_{\mathbb{Z}_{p}} \mathbb{F}_{p}\right)$ l'invariant de Hasse. C'est la différentielle du morphisme de Vershiebung $V: E^{(p)} \rightarrow E$ défini relativement à $X \times_{\operatorname{Spec} \mathbb{Z}_{p}}$ Spec $\mathbb{F}_{p}$ (voir également les rappels de la section A.1). Remarquons que $\mathrm{Ha}^{p^{n}}$ se relève canoniquement en une section de $\mathrm{H}^{0}\left(\mathfrak{X}, \omega_{E}^{(p-1) p^{n}} \otimes_{\mathbb{Z}_{p}} \mathbb{Z} / p^{n+1} \mathbb{Z}\right)$. En effet, si $\tilde{\mathrm{Ha}} \mathrm{a}_{1}$ et $\tilde{\mathrm{Ha}}{ }_{2}$ sont deux relèvements locaux à $\mathbb{Z}_{p}$ de Ha, la formule du binôme montre que $\left.(\tilde{\mathrm{Ha}})_{1}\right)^{p^{n}}=(\tilde{\mathrm{Ha}})^{p^{n}}$ modulo $p^{n+1}$. Soit $\operatorname{Hdg}:=\operatorname{Hdg}(E) \subset \mathscr{O}_{\mathfrak{X}}$ l'idéal de Hodge engendré par $p$ et un relèvement local quelconque de $\operatorname{Ha}(E) \omega_{\mathrm{E}}^{1-p}$ (voir le numéro A.1). Soit $A_{0}$ un anneau intègre, $\alpha \in A_{0}$ un élément non nul tel que $A_{0}$ soit $\alpha$-adiquement complet. On suppose aussi que $p \in \alpha A_{0}$. Remarquons qu'avec ces hypothèses $A_{0}$ est canoniquement une $\mathbb{Z}_{p}$-algèbre.

Soit $\mathfrak{Y}$ le schéma formel sur $\operatorname{Spf} A_{0}$ obtenu en changeant de base $\mathfrak{X}$ de $\operatorname{Spf} \mathbb{Z}_{p}$ à $\operatorname{Spf} A_{0}$. On note $\mathfrak{Y}_{\text {ord }}$ l'ouvert lieu ordinaire de $\mathfrak{Y}$, défini par la condition : Ha inversible. 
Définition 3.1. - Pour tout entier $r \in \mathbb{N}$, soit $\mathfrak{Y}_{r}$ le foncteur qui à toute $A_{0}$-algèbre $\alpha$-adiquement complète et sans $\alpha$-torsion $R$ associe les classes d'équivalence de couples $\left(f: \operatorname{Spf} R \rightarrow \mathfrak{X}, \eta \in \mathrm{H}^{0}\left(\operatorname{Spf} R, f^{\star} \omega^{(1-p) p^{r+1}}\right)\right)$ tels que

$$
\mathrm{Ha}^{p^{r+1}} \eta=\alpha \bmod p^{2} \text {. }
$$

Deux couples $(f, \eta)$ et $\left(f^{\prime}, \eta^{\prime}\right)$ sont équivalents si $f=f^{\prime}$ et $\eta=\eta^{\prime}\left(1+\frac{p^{2}}{\alpha} u\right)$ pour un élément $u \in R$.

Proposition 3.1. - Le foncteur $\mathfrak{Y}_{r}$ est représentable par un schéma formel sans $\alpha$-torsion qui est un ouvert d'un éclatement admissible de $\mathfrak{Y}$.

Démonstration. - Soit $\operatorname{Spf} B$ un ouvert affine de $\mathfrak{Y}$ sur lequel le faisceau $\omega_{E}$ est trivial. Identifions Ha avec un scalaire et soit $\tilde{\mathrm{Ha}} \in B$ un relèvement de Ha. L'image inverse de $\operatorname{Spf} B$ dans $\mathfrak{Y}_{r}$ vaut $\operatorname{Spf} B\langle X\rangle /\left(\tilde{\mathrm{Ha}}{ }^{p^{r+1}} X-\alpha\right)$.

Lemme 3.1. - L'idéal de Hodge Hdg est localement libre sur $\mathfrak{Y}_{r}$ et $\alpha \in \mathrm{Hdg}$.

Démonstration. - Localement sur $\mathfrak{Y}_{r}$, on a $\mathrm{Ha}^{p^{r+1}} \eta=\alpha \bmod p^{2}$. Il en résulte que $\alpha$ et donc $p$ appartiennent à $\mathrm{Hdg}^{p^{r+1}}$. On peut appliquer le lemme A.1 pour conclure à la liberté de Hdg.

La théorie du sous-groupe canonique est discutée en détail dans l'appendice A.

Proposition 3.2. - Soit $k \in \mathbb{Z}_{\geq 0}$. Supposons que $p \in \alpha^{p^{k}} A_{0}$. Sur $\mathfrak{Y}_{r}$ on dispose pour tout $n \leq r+k$ d'un sous-groupe canonique $H_{\mathrm{n}} \hookrightarrow E\left[p^{n}\right]$ qui est localement libre de rang $p^{\mathrm{n}}$. La réduction de $H_{n}$ modulo $p \mathrm{Hdg}^{-\frac{p^{n}-1}{p-1}}$ coüncide avec le noyau de Frobenius à la puissance $n$.

Démonstration. - Le schéma formel $\mathfrak{Y}_{r}$ est la réunion de l'ouvert ordinaire et de l'ouvert «de bonne réduction» où le schéma semi-abélien $E$ est un schéma abélien. Sur l'ouvert de bonne réduction, le groupe $E\left[p^{\infty}\right]$ est un Barsotti-Tate et on peut donc utiliser le corollaire A.2. Sur l'ouvert ordinaire, on définit $H_{n}$ comme la composante neutre du schéma quasi-fini $E\left[p^{n}\right]$. Ces deux constructions sont compatibles.

Proposition 3.3. - L'isogénie " diviser par le sous-groupe canonique »: $E \rightarrow E / H_{1}$, induit un morphisme fini et plat de degré $p, \phi: \mathfrak{Y}_{r} \rightarrow \mathfrak{Y}_{r-1}$ pour tout $r \geq 1$. Ce morphisme vérifie $\phi^{\star}\left(E_{\left.\right|_{\mathfrak{Y}_{r-1}}}\right)=\left(E_{\left.\right|_{\mathfrak{Y} r}}\right) / H_{1}$.

Démonstration. - Soit $(f, \eta)$ un $R$-point de $\mathfrak{Y}_{r}$. Le morphisme $f$ est donné par un schéma semi-abélien $E \rightarrow \operatorname{Spec} R$ et une structure de niveau $N$, notée $\psi_{N}$. Soit $E^{\prime}=E / H_{1}$ et $\psi_{N}^{\prime}$ la structure de niveau $N$ induite sur $E^{\prime}$. Au couple $\left(E^{\prime}, \psi_{\mathrm{N}}^{\prime}\right)$ correspond un nouveau morphisme $f^{\prime}:$ Spf $R \rightarrow \mathfrak{Y}$. D'autre part, on a la relation $\mathrm{Ha}\left(E^{\prime}\right)=\mathrm{Ha}(E)^{p} \bmod \frac{p}{\mathrm{Hdg}}$. Ici on utilise le fait que $E^{\prime}=E^{(p)} \bmod \frac{p}{\mathrm{Hdg}}$ et donc que $\omega_{E^{\prime}} \simeq \omega_{\mathrm{E}}^{p} \bmod \frac{p}{\mathrm{Hdg}}$. En élevant à la puissance $p^{\mathrm{r}}$ on obtient: $\mathrm{Ha}\left(E^{\prime}\right)^{p^{\mathrm{r}}}=\mathrm{Ha}(E)^{p^{r+1}} \bmod \left(\left(\frac{p}{\operatorname{Hdg}(E)}\right)^{p^{r}}, p\left(\frac{p}{\operatorname{Hdg}(E)}\right)\right)$. Il en résulte que $\operatorname{Ha}\left(E^{\prime}\right)^{p^{\mathrm{r}} \eta}=\alpha \bmod \left(\left(\frac{p}{\operatorname{Hdg}(E)}\right)^{p^{r}}, p\left(\frac{p}{\operatorname{Hdg}(E)}\right)\right)$. Comme $\left(\left(\frac{p}{\operatorname{Hdg}(E)}\right)^{p^{r}}, p\left(\frac{p}{\operatorname{Hdg}(E)}\right)\right) \subset$ $\frac{p}{\operatorname{Hdg}(E)} \operatorname{Hdg}(E)^{p^{r+1}}$ il existe alors $\tilde{\eta} \in \omega_{E^{\prime}}^{(p-1) p^{\mathrm{r}}}$ qui relève $\eta \bmod \frac{p}{\operatorname{Hdg}(E)}$ tel que $\operatorname{Ha}\left(E^{\prime}\right)^{p^{\mathrm{r}}} \tilde{\eta}=$ $\alpha \bmod p^{2}$. Le morphisme cherché est donc $(f, \eta) \mapsto\left(f^{\prime}, \tilde{\eta}\right)$. Soit Spf $B$ un ouvert affine de $\mathfrak{Y}$, soit $\operatorname{Spf} B\langle X\rangle /\left(\tilde{\mathrm{Ha}}{ }^{p^{\mathrm{r}}} X-\alpha\right)$ son image inverse dans $\mathfrak{Y}_{r-1}$, et soit $\operatorname{Spf} B\langle X\rangle /\left(\tilde{\mathrm{Ha}}^{p^{r+1}} X-\alpha\right)$ 
son image inverse dans $\mathfrak{Y}_{\mathrm{r}}$. Le morphisme $\phi$ induit sur ces anneaux un morphisme $\phi: B\langle X\rangle /\left(\tilde{\mathrm{Ha}}{ }^{p^{\mathrm{r}}} X-\alpha\right) \rightarrow B\langle X\rangle /\left(\tilde{\mathrm{Ha}}{ }^{p^{r+1}} X-\alpha\right)$ qui est $A_{0}$-linéaire. La réduction de $\phi$ modulo $\frac{p}{\tilde{H} \mathrm{a}}$ est le morphisme de Frobenius (relativement à $\left.A_{0}\right) \operatorname{sur} B /\left(\frac{p}{\tilde{\mathrm{H} a}}\right)$ et $\phi(X)=X \bmod \frac{p}{\tilde{\mathrm{Ha}}}$. Ainsi, le morphisme $\phi$ est fini et plat de degré $p$ modulo $\frac{p}{\mathrm{Hdg}}$. Comme tous les schémas formels en jeu sont sans $A_{0}$-torsion, il en résulte que le morphisme est fini et plat.

\subsection{Tour d'Igusa partielle}

L'existence du sous-groupe canonique permet de construire des revêtements des schémas formels $\mathfrak{Y}_{r}$.

3.2.1. Construction. - On suppose désormais que $A_{0}$ est une complétion d'une $\mathbb{Z}_{p}$-algèbre de type fini. Remarquons que cette hypothèse implique que $A_{0}$ est noethérien. Soit $A=$ $A_{0}[1 / \alpha]$ et $A^{+}$le normalisé de $A_{0}$ dans $A$. Au schéma formel $\operatorname{Spf} A_{0}$ on peut associer un espace adique analytique $\operatorname{Spa}\left(A, A^{+}\right)$. Le caractère noethérien de $A_{0}$ nous assure que $\operatorname{Spa}\left(A, A^{+}\right)$est bien un espace adique ([19], Thm. 2.2). D'après le théorème principal de [30], on déduit également que $\operatorname{Spf} A_{0}$ et $\mathfrak{Y}_{r}$ sont des schémas formels excellents. Nous rappelons la proposition suivante, qui utilise l'excellence. Elle est utile pour définir des schémas formels par normalisation :

Proposition 3.4. - Soit $R$ un anneau normal, excellent et I un idéal de $R$. Soit $f \in R$. Alors la complétion I-adique de $R\left[f^{-1}\right]$ est normale.

Démonstration. - L'anneau $R\left[f^{-1}\right]$ est excellent et normal. Notons $\widehat{R\left[f^{-1}\right]}$ sa complétion $I$-adique. Le morphisme $R\left[f^{-1}\right] \rightarrow \widehat{R\left[f^{-1}\right]}$ est régulier. Comme $R\left[f^{-1}\right]$ est normal, il en va de même de $\widehat{R\left[f^{-1}\right.}$. Voir [16], EGA IV, 7.8.3, v.

Au schéma formel $\mathfrak{Y}_{r}$ on associe l'espace adique analytique $\mathscr{Y}_{r}=\mathfrak{Y}_{r}^{\mathrm{ad}} \times_{\operatorname{Spa}\left(A_{0}, A_{0}\right)}$ $\operatorname{Spa}\left(A, A^{+}\right)$où $\mathfrak{Y}_{r}^{\text {ad }}$ désigne l'adification du schéma formel $\mathfrak{Y}_{r}$ ([19], sect. 4). Soit $k \in \mathbb{Z}_{\geq 0}$. On suppose que $\alpha^{p^{k}} \mid p$. Pour tout $n \leq r+k$, on dispose, d'après la proposition 3.2, d'un sous-groupe canonique $H_{n} \rightarrow \mathfrak{Y}_{r}$ d'échelon $n$. De plus, le groupe $H_{n}^{D}$ est étale au-dessus de la fibre analytique $\mathscr{Y}_{r}$ (grâce au point 6. du corollaire A.2 et au lemme 3.1). Soit $\mathscr{T} \mathscr{G}_{n, r} \rightarrow \mathcal{Y}_{r}$ le revêtement fini étale qui paramètre les isomorphismes $\mathbb{Z} / p^{n} \mathbb{Z} \rightarrow H_{n}^{D}$. C'est un revêtement galoisien de groupe $\left(\mathbb{Z} / p^{n} \mathbb{Z}\right)^{\times}$qu'on appelle la tour d'Igusa partielle analytique de niveau $n$. On définit alors un schéma formel $\mathfrak{I G}_{n, r} \rightarrow \mathfrak{Y}_{r}$ comme la normalisation de $\mathfrak{Y}_{r}$ dans $\mathscr{G} \mathscr{G}_{n, r}$. On appelle $\mathfrak{I G}_{n, r}$ la tour d'Igusa partielle formelle de niveau $n$.

Lemme 3.2. - La normalisation $\mathfrak{I G}_{n, r}$ est bien définie. Le morphisme $\mathfrak{I G}_{n, r} \rightarrow \mathfrak{Y}_{r}$ est fini.

Démonstration. - Soit $\operatorname{Spf} R$ un ouvert formel de $\mathfrak{Y}_{r}$. Soit $R^{\prime}[1 / \alpha]$ (la notation est ambiguë pour l'instant car $R^{\prime}$ n'est pas encore défini) l'anneau de $\mathscr{J} \mathscr{G}_{n, r}$ au-dessus de $\operatorname{Spa}(R, R)^{\text {an }}$. Par hypothèse $R^{\prime}[1 / \alpha]$ est une $R[1 / \alpha]$-algèbre finie étale. Soit $R^{\prime}$ le normalisé de $R$ dans $R[1 / \alpha]$. Le caractère excellent de $R$ nous assure que $R^{\prime}$ est finie sur $R$. L'ouvert de $\mathfrak{I G}_{n, r}$ au-dessus de $\operatorname{Spf} R$ est par définition $\operatorname{Spf} R^{\prime}$. Pour voir que la construction a un sens, nous devons montrer que si $f \in R$ alors $R^{\prime}\langle 1 / f\rangle$ est le normalisé de $R\langle 1 / f\rangle$ dans $R^{\prime}[1 / \alpha] \otimes_{\mathrm{R}} R\langle 1 / f\rangle$ (qui est l'anneau des fonctions sur la fibre dans $\mathscr{J} \mathscr{G}_{n, r}$ 
de $\left.\operatorname{Spa}(R\langle 1 / f\rangle, R\langle 1 / f\rangle)^{\text {an }}\right)$. Comme $R\langle 1 / f\rangle$ est plat sur $R, R^{\prime}\langle 1 / f\rangle=R^{\prime} \otimes_{R} R\langle 1 / f\rangle$ est bien un sous-anneau de $R^{\prime}[1 / \alpha] \otimes_{\mathrm{R}} R\langle 1 / f\rangle$. Il est normal d'après la proposition 3.4 et fini sur $R\langle 1 / f\rangle$.

Le groupe $\left(\mathbb{Z} / p^{n} \mathbb{Z}\right)^{\times}$agit sur $\mathfrak{I G}_{n, r}$ à travers son action sur le morphisme $\mathbb{Z} / p^{n} \mathbb{Z} \rightarrow H_{n}^{D}$. Un élément $a \in\left(\mathbb{Z} / p^{n} \mathbb{Z}\right)^{\times}$agit sur le morphisme $\mathbb{Z} / p^{n} \mathbb{Z} \rightarrow H_{n}^{D}$ en le précomposant par la multiplication par $a$. On possède des morphismes d'oubli évidents $\mathfrak{I G}_{n, r} \rightarrow \mathfrak{I G}_{n-1, r}$. Les différentes tours d'Igusa partielles s'organisent en une suite

$$
\mathfrak{I G}_{r+k, r} \rightarrow \mathfrak{I G}_{r+k-1, r} \rightarrow \cdots \rightarrow \mathfrak{Y}_{r}
$$

de morphismes finis, équivariants pour l'action de $\mathbb{Z} / p^{r+k} \mathbb{Z}^{\times}$. Le lemme suivant ne pose pas de problème.

Lemme 3.3. - Pour tout $n \geq 2$, soit $h_{n}: \mathfrak{I G}_{n, r} \rightarrow \mathfrak{I G}_{n-1, r}$ le morphisme structural. Alors

$$
\left.\left(\left(h_{n}\right)_{\star} \mathscr{O}_{\mathfrak{I} \mathfrak{G}_{n, r}}\right)\right)^{1+p^{n-1} \mathbb{Z} / p^{n} \mathbb{Z}}=\mathscr{O}_{\mathfrak{I G} \mathfrak{G}_{n-1, r}} .
$$

Soit $h_{1}: \mathfrak{I G}_{1, r} \rightarrow \mathfrak{Y}_{r}$. Alors

$$
\left.\left(\left(h_{1}\right)_{\star} \mathscr{O}_{\mathfrak{I} \mathfrak{G}_{1, r}}\right)\right)^{\mathbb{Z} / p \mathbb{Z}^{\times}}=\mathscr{O}_{\mathfrak{Y}_{\mathrm{r}}} .
$$

3.2.2. Ramification de la tour d'Igusa partielle. - Le morphisme $h: \mathfrak{I G}_{n, r} \rightarrow \mathfrak{I G}_{n-1, r}$ est fini et étale en fibre analytique. Grâce au lemme 3.3, on a donc une trace $\operatorname{Tr}_{\mathfrak{I G}}: h_{\star} \mathscr{O}_{\mathfrak{I G}, r} \rightarrow$ $\mathscr{O}_{\mathfrak{I} \mathfrak{G}_{n-1, r}}$. La source et le but du morphisme $\operatorname{Tr}_{\mathfrak{I G}}$ dépendent bien sûr de $n$ et de $r$, mais nous l'omettons de la notation.

Proposition 3.5. - Pour tout $n \geq 2$, on a

$$
\operatorname{Hdg}^{p^{n-1}} \mathscr{O}_{\mathfrak{I} \mathfrak{G}_{n-1, r}} \subset \operatorname{Tr}_{\mathfrak{I G}}\left(h_{\star} \mathscr{O}_{\mathfrak{I} \mathfrak{G}_{n, r}}\right) .
$$

Pour $n=1, \operatorname{Tr}_{\mathfrak{I G}}\left(h_{\star} \mathscr{O}_{\mathfrak{I G}}\right)=\mathscr{O}_{\mathfrak{Y Y}_{r}}$.

Démonstration. - Pour $n=1$, l'énoncé est clair car le morphisme est de degré $p-1$ premier à $p$. Soit $\operatorname{Spf} R$ un ouvert affine de $\mathfrak{Y}_{r}$. Supposons que Hdg est trivial sur cet ouvert, engendré par Ha. Soit Spf $B_{n}$ et $\operatorname{Spf} B_{n-1}$ les fibres de $H_{n}^{D}$ et $H_{n-1}^{D}$ au-dessus de $\operatorname{Spf} R$. Soit Spf $C$ la fibre du quotient $\left(H_{n} / H_{n-1}\right)^{D}$ sur Spf $R$. Le morphisme $H_{n}^{D} \rightarrow H_{n-1}^{D}$ est un espace homogène sous $\left(H_{n} / H_{n-1}\right)^{D}$ pour la topologie $f p p f$. Soit $D_{B_{n} / B_{n-1}}$ la différente du morphisme $B_{n-1} \rightarrow B_{n}$ et $D_{C / R}$ celle du morphisme $R \rightarrow C$. On a l'égalité :

$$
D_{B_{n} / B_{n-1}} \otimes_{B_{n-1}} B_{n}=D_{C / R} \otimes_{\mathrm{R}} B_{n}
$$

de $B_{n} \otimes_{B_{n-1}} B_{n}=C \otimes_{R} B_{n}$-modules. Par ailleurs, on a $D_{C / R}=\operatorname{Hdg}^{p^{n-1}} C$ d'après le corollaire A.2 et le lien entre discriminant et différente (voir [14], sect. 1 et 2.). Par descente fidèlement plate, il en résulte que $D_{B_{n} / B_{n-1}}=\operatorname{Hdg}^{p^{n-1}} B_{n}$. On a un isomorphisme $D_{B_{n} / B_{n-1}}^{-1} \rightarrow \operatorname{Hom}_{B_{n-1}}\left(B_{n}, B_{n-1}\right)$ donné par $x \mapsto \operatorname{Tr}_{B_{n} / B_{n-1}}(x \cdot)$. Vérifions que l'idéal $\operatorname{Tr}_{B_{n} / B_{n-1}}\left(D_{B_{n} / B_{n-1}}^{-1}\right) \subset B_{n-1}$ est $B_{n-1}$. La propriété est locale pour la topologie de Zariski sur Spec $B_{n-1}$ donc on peut supposer que $B_{n}$ est un module libre sur $B_{n-1}$. On possède donc une application surjective $B_{n} \rightarrow B_{n-1}$ de $B_{n-1}$-modules qui s'écrit $\operatorname{Tr}_{B_{n} / B_{n-1}}(x$.) pour $x \in D_{B_{n} / B_{n-1}}^{-1}$ et donc $\operatorname{Tr}_{B_{n} / B_{n-1}}\left(D_{B_{n} / B_{n-1}}^{-1}\right)=B_{n-1}$. Par conséquent, on peut trouver un élément $d_{n} \in B_{n}$ tel que $\operatorname{Tr}_{B_{n} / B_{n-1}}\left(d_{n}\right)=\tilde{\mathrm{Ha}}^{p^{n-1}}$. Pour tout $n \geq 1$, on a une immersion 
ouverte de $\mathscr{Y}_{r}$-espaces adiques analytiques $\mathscr{J} \mathscr{G}_{n, r} \hookrightarrow H_{n}^{D}$. De plus, pour tout $n \geq 2$, on a un diagramme cartésien au-dessus de $\mathscr{y}_{r}$ :

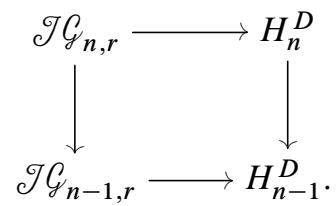

En utilisant la normalité de $\mathfrak{I G}_{n, r}$ et la finitude de $H_{n}^{D} \rightarrow \mathfrak{Y}_{r}$ on obtient un diagramme commutatif :

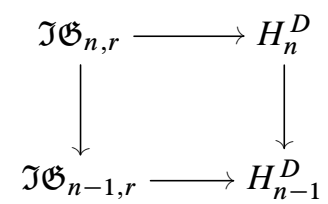

au-dessus de $\mathfrak{Y}_{r}$. On peut donc tirer les sections $d_{n}$ en des éléments $d_{n}^{\prime} \in \mathscr{O}_{\mathfrak{I G}_{n, r}}(\operatorname{Spf} R)$ qui vérifient $\operatorname{Tr}_{\mathfrak{I G}}\left(d_{n}^{\prime}\right)=\tilde{\mathrm{Ha}}{ }^{p^{n-1}}$.

Corollaire 3.1. - Soit $\operatorname{Spf} R$ un ouvert de $\mathfrak{Y}_{r}$ au-dessus duquel le faisceau Hdg est trivial, engendré par unélément $\tilde{\mathrm{H}}$ a. Il existe alors des éléments $c_{0}=1$ et $c_{n} \in \tilde{\mathrm{Ha}}^{-\frac{p^{n}-p}{p-1}} \mathscr{O}_{\mathfrak{I G}}(\operatorname{Spf} R)$ pour tout $1 \leq n \leq r+k$ tels que $\operatorname{Tr}_{\mathfrak{I G}}\left(c_{n}\right)=c_{n-1}$.

Démonstration. - On a $c_{0}=1, c_{1}=\frac{1}{p-1}$. Supposons $c_{n-1}$ construit. D'après la proposition précédente, $\tilde{\mathrm{Ha}}^{\frac{p^{n-1}-p}{p-1}} c_{n-1} \in \tilde{\mathrm{Ha}}{ }^{-p^{n-1}} \operatorname{Tr}_{\mathfrak{I} \mathfrak{G}}\left(\mathscr{O}_{\mathfrak{I} \mathfrak{G}_{n, r}}(\operatorname{Spf} R)\right)$. On peut donc trouver $c_{n}$ qui convient.

3.2.3. Fonctorialité pour le Frobenius. - On suppose toujours que $p \in \alpha^{p^{k}} A_{0}$ et $n \leq r+k$. Rappelons qu'on possède un morphisme de Frobenius $\phi: \mathfrak{Y}_{r+1} \rightarrow \mathfrak{Y}_{r}$ (proposition 3.3).

Proposition 3.6. - On possède un morphisme fini, $\left(\mathbb{Z} / p^{n} \mathbb{Z}\right)^{\times}$-équivariant, $\phi: \mathfrak{I G}_{n, r+1} \rightarrow \mathfrak{I G}_{n, r}$, au-dessus du morphisme précédent.

Démonstration. - Commençons par construire un morphisme $\phi: \mathscr{T}_{\mathscr{G}_{n, r+1}} \rightarrow \mathscr{T}_{\mathscr{G}_{n, r}}$. Soit $\left(R, R^{+}\right)$une algèbre affinoïde et $x: \operatorname{Spa}\left(R, R^{+}\right) \rightarrow \mathscr{J}_{g, r+1}$ un point. Soit $E \rightarrow R$ le schéma semi-abélien correspondant. Pour tout $m \leq k+r+1$, il possède un sous-groupe canonique $H_{m}$ d'échelon $m$ ainsi qu'une trivialisation $\psi: \mathbb{Z} / p^{n} \mathbb{Z} \rightarrow H_{n}^{D}$. Soit $E^{\prime}=E / H_{1}$ et $H_{n}^{\prime}$ le sous-groupe canonique de $E^{\prime}$ d'échelon $n$. On a un isomorphisme canonique (qui existe aussi au niveau entier, voir le corollaire A.2) $H_{n}^{\prime} \simeq H_{n+1} / H_{1}$. Par ailleurs, la multiplication par $p$ dans $H_{n+1}$ se factorise en un isomorphisme $H_{n+1} / H_{1} \simeq H_{\mathrm{n}}$ (qui n'est pas un isomorphisme entier en général). On possède donc un isomorphisme $H_{n}^{\prime} \simeq H_{n}$ et en composant le dual de cet isomorphisme et $\psi$, on obtient la trivialisation $\psi^{\prime}: \mathbb{Z} / p^{n} \mathbb{Z} \rightarrow\left(H_{n}^{\prime}\right)^{D}$. Le morphisme $\phi$ associe à $(E, \psi)$ le couple $\left(E^{\prime}, \psi^{\prime}\right)$. Par normalisation, le morphisme se prolonge au niveau entier.

\subsection{Application et notation}

Ce numéro rassemble la plupart des notations utilisées dans l'article. 
3.3.1. L'espace des poids. - Soit $I=\left[p^{k}, p^{k^{\prime}}\right]$ avec $k, k^{\prime} \in \mathbb{Z}_{\geq 0} \cup\{+\infty\}$ ou $I=[0,1]$. On note $B_{I}=\mathrm{H}^{0}\left(\mathscr{W}_{I}^{0}, \mathscr{O}_{\mathscr{W}^{0}}^{+}\right)$. Explicitons l'anneau $B_{I}$ (on pourra utiliser le critère de Serre pour vérifier que ces anneaux sont intégralement clos) :

1. si $k, k^{\prime} \in \mathbb{Z}_{\geq 0}, B_{I}=\mathbb{Z}_{p}[[T]]\langle u, v\rangle /\left(T^{p^{\mathrm{k}}} v-p, u v-T^{p^{k^{\prime}}-p^{k}}\right)$,

2. si $k \in \mathbb{Z}_{\geq 0}, k^{\prime}=+\infty, B_{I}=\mathbb{Z}_{p}[[T]]\langle u\rangle /\left(p-u T^{p^{k}}\right)$,

3. si $k, k^{\prime}=+\infty, B_{I}=\mathbb{F}_{p}[[T]]$,

4. si $I=[0,1], B_{I}=\mathbb{Z}_{p}[[T]]\langle v\rangle /(T-v p)$.

Soit $\mathfrak{W}_{I}^{0}=\operatorname{Spf} B_{I}$ avec $B_{I}$ muni de la topologie $(p, T)$ adique. La fibre analytique de $\mathfrak{W}_{I}^{0}$ est $\mathscr{W}_{I}^{0}$. On note $\mathfrak{W}_{I}=\operatorname{Spf} B_{I}\left[\mathbb{Z} / q \mathbb{Z}^{\times}\right]$. Sa fibre analytique vaut $\mathscr{W}_{I}$.

3.3.2. Les courbes modulaires. - On rappelle que $\mathfrak{X}$ est la courbe modulaire formelle sur Spf $\mathbb{Z}_{p}$. Pour tout intervalle rationnel $I \subset[0, \infty]$, on note $\mathscr{C}_{I}=\mathfrak{X} \times_{\operatorname{Spf}} \mathbb{Z}_{p} \mathscr{W}_{I}^{0}$ et $\mathcal{M}_{I}=\mathfrak{X} \times_{\mathrm{Spf} \mathbb{Z}_{p}} \mathscr{W}_{I}$. On note $\mathcal{M}_{\text {ord,I }}$ (resp. $\mathscr{C}_{\text {ord,I }}$ ) l'ouvert ordinaire de $\mathcal{M}_{I}$ (resp. ${ }^{\mathfrak{o}}{ }_{I}$ ) défini par $|\mathrm{Ha}|=1$.

Pour tout $r \in \mathbb{N}$, soit $\mathscr{M}_{r, I}$ (resp. $\mathscr{C}_{r, I}$ ) l'ouvert de $\mathcal{M}_{I}$ (resp. $\mathscr{C}_{I}$ ) donné par la condition :

$$
\left|\tilde{\mathrm{Ha}}^{p^{r+1}}\right| \geq \sup \{|p|,|T|\}
$$

où Ha désigne un relèvement local de l'invariant de Hasse.

Remarque 3.1. - On peut penser à l'ouvert $M_{r, I}$ comme à une famille de voisinages

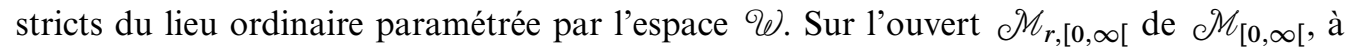
mesure qu'on s'approche du bord de l'espace des poids, les voisinages vus pour la topologie $p$-adique se rétrécissent. Par contre, si on regarde les voisinages pour la topologie $T$-adique au voisinage du bord de l'espace des poids, ils ont un rayon constant!

Pour tout intervalle $I$ comme dans le paragraphe précédent, on note $\mathfrak{X}_{I}=\mathfrak{X} \times \times_{\operatorname{Spf}} \mathbb{Z}_{p} \mathfrak{W}_{I}^{0}$ et $\mathfrak{M}_{I}=\mathfrak{X} \times_{\operatorname{Spf} \mathbb{Z}_{p}} \mathfrak{W}_{I}$. On applique la construction du paragraphe 3.1 avec $A_{0}=B_{I}$ et $\alpha=T$ dans les cas où $I=\left[p^{k}, p^{k^{\prime}}\right]$ avec $k \geq 0$, et avec $\alpha=p$ si $I=[0,1]$. On note $\mathfrak{X}_{r, I}:=\mathfrak{Y}_{r}$ pour ces choix. C'est donc un ouvert d'un éclaté de $\mathfrak{X}_{I}$. On remarque que $\mathfrak{C}_{r, I}$ est l'espace adique fibre analytique de $\mathfrak{X}_{r, I}$. On note $\mathfrak{M}_{r, I}=\mathfrak{X}_{r, I} \times_{\mathfrak{W}_{I}^{0}} \mathfrak{W}_{I}$, et on observe que $\mathcal{M}_{r, I}$ est sa fibre analytique.

Proposition 3.7. - Pour tout $n \leq r+k$, on possède un sous-groupe canonique $H_{n}$ d'ordre $n$ sur $\mathfrak{X}_{r, I}$ et $\mathfrak{M}_{r, I}$. L'isogénie $\bar{E} \rightarrow E / H_{1}$ nous fournit des morphismes finis et plats de Frobenius relatif:

$$
\phi: \mathfrak{X}_{r+1, I} \rightarrow \mathfrak{X}_{r, I} \text { et } \phi: \mathfrak{M}_{r+1, I} \rightarrow \mathfrak{M}_{r, I}
$$

Démonstration. - Cela résulte des propositions 3.2 et 3.3. 
3.3.3. La tour d'Igusa. - On vérifie grâce au théorème principal de [30] que $\mathfrak{X}_{r, I}$ est excellent. Vérifions qu'il est normal.

Lemme 3.4. - Le schéma formel $\mathfrak{X}_{r, I}$ est normal.

Démonstration. - Traitons simplement le cas où $I=\left[p^{\mathrm{k}}, p^{k^{\prime}}\right]$. Les autres cas sont similaires. Soit $\operatorname{Spf} A$ un ouvert de la courbe modulaire sur Spf $\mathbb{Z}_{p}$. Supposons que $\omega_{E}$ est trivial sur $A$ et soit $\tilde{\mathrm{Ha}} \in A$ un relèvement de l'invariant de Hasse vu comme un scalaire. Soit

$$
B=A[[T]]\langle u, v, w\rangle /\left(T^{p^{\mathrm{k}}} v-p, u v-T^{p^{k^{\prime}}-p^{k}}, \tilde{\mathrm{Ha}}^{p^{r+1}} w-T\right) .
$$

Alors $\operatorname{Spf} B$ est l'image inverse de $\operatorname{Spf} A$ dans $\mathfrak{X}_{r, I}$. Pour vérifier qu'il est normal, appliquons le critère de Serre. On voit facilement que $B$ est une intersection complète dans $A[[T]]\langle u, v, w\rangle$ donc il est Cohen-Macaulay. Vérifions que $B$ est régulier en codimension 1. Soit $x \in \operatorname{Spec} B$ de codimension 1. Si $T \notin x$ alors $x \in$ Spec $B[1 / T]$ et $B[1 / T]$ est formellement lisse (car c'est l'anneau des fonctions sur la fibre rigide de $\operatorname{Spf} B$ qui est lisse). Si $T \in x$ alors $x$ est un point générique de $B / T B=(A / p[u, v, w]) /\left(u v, \tilde{\mathrm{Ha}}^{p^{r+1}} w\right)$. Si Ha $\notin x$, alors $B_{x} / T B_{x}$ est réduit et donc un corps. Ainsi $B_{x}$ est régulier de dimension 1 et son paramètre est $T$. Si Ha $\in x$ alors $B / \tilde{\mathrm{Ha}} B=A /(p, \tilde{\mathrm{Ha}})[u, v, w] /(u v)$ est réduit car Ha a des zéros simples dans $A / p$. Ainsi $B_{x}$ est régulier de dimension 1 et son paramètre est $\tilde{\mathrm{H} a}$.

On dispose sur $\mathfrak{X}_{r, I}$ d'un sous-groupe canonique d'échelon $n \leq k+r$ dès que $I \subset\left[p^{k}, \infty\right]$. On peut alors définir la tour d'Igusa partielle formelle de niveau $n$ grâce au numéro 3.2, $\mathfrak{I G}_{n, r, I} \rightarrow \mathfrak{X}_{r, I}$. On note $\mathscr{J} \mathcal{G}_{n, r, I}$ l'espace adique fibre analytique de $\mathfrak{I G}_{n, r, I}$. C'est la tour d'Igusa partielle analytique de niveau $n$.

\section{Formes modulaires surconvergentes en caractéristique $p$}

Dans cette partie, nous construisons des espaces de formes surconvergentes en caractéristique $p$.

\subsection{Courbe modulaire en caractéristique $p$}

Soit $\bar{X} \rightarrow$ Spec $\mathbb{F}_{p}$ la courbe modulaire compactifiée de niveau $N$ premier à $p$, soit Ha l'invariant de Hasse et Hdg l'idéal de Hodge. Soit $\bar{X}_{\text {ord }}$ l'ouvert ordinaire. On rappelle que $\mathfrak{W}_{\{\infty\}}^{0}=\operatorname{Spf} \mathbb{F}_{p}[[T]]$. On note $\bar{\kappa}: \mathbb{Z}_{p}^{\times} \rightarrow \mathbb{F}_{p}[[T]]^{\times}$le caractère qui est trivial sur $\mathbb{Z} / q \mathbb{Z}^{\times}$et qui envoie $\exp (q)$ sur $1+T$. Pour tout caractère $\chi: \mathbb{Z} / q \mathbb{Z}^{\times} \rightarrow \mathcal{O}_{\mathbb{C}_{p}}^{\times}$, on note $\bar{\chi}$ sa réduction modulo $\mathfrak{m}_{\mathscr{C}_{p}}$ et $\bar{\kappa}_{\chi}$ le caractère $\bar{\kappa} \otimes \bar{\chi}$.

Soit $\mathfrak{X}_{\{\infty\}}=\bar{X} \times_{\text {Spec } \mathbb{F}_{p}} \mathfrak{W}_{\{\infty\}}^{0}$ et $\mathfrak{X}_{\text {ord },\{\infty\}}=\bar{X}_{\text {ord }} \times_{\text {Spec } \mathbb{F}_{p}} \mathfrak{W}_{\{\infty\}}^{0}$ On a construit (voir les numéros 3.1 et 3.3) un schéma formel $\mathfrak{X}_{r,\{\infty\}} \rightarrow \mathfrak{X}_{\{\infty\}}$ pour tout entier $r \geq 0$. Soit $\mathfrak{C}_{\{\infty\}}$, $\mathscr{C}_{r,\{\infty\}}$ les espaces adiques sur $\mathscr{W}_{\{\infty\}}^{0}=\operatorname{Spa}\left(\mathbb{F}_{p}((T)), \mathbb{F}_{p}[[T]]\right)$, fibres génériques de $\mathfrak{X}_{\{\infty\}}$ et $\mathscr{C}_{r,\{\infty\}}$. Ainsi, $\mathscr{C}_{r,\{\infty\}}$ est l'ouvert de $\mathscr{C}_{\{\infty\}}$ d'équation $\left|\mathrm{Ha}^{p^{r+1}}\right| \geq|T|$.

Comme on est en caractéristique $p$, on dispose sur tous ces espaces, pour tout $n \geq 0$, d'un sous-groupe canonique $H_{n}$ d'ordre $n$. C'est le noyau de l'isogénie de Frobenius itérée $n$ fois : $F^{n}: E \rightarrow E^{\left(p^{n}\right)}$. 
La règle qui associe à $E$ son quotient $E / H_{1}$ nous fournit des morphismes Frobenius relatif $\mathfrak{X}_{\{\infty\}} \rightarrow \mathfrak{X}_{\{\infty\}}, \mathfrak{X}_{\text {ord },\{\infty\}} \rightarrow \mathfrak{X}_{\text {ord },\{\infty\}}$ et pour tout $r \geq 0, \mathfrak{X}_{r+1,\{\infty\}} \rightarrow \mathfrak{X}_{r,\{\infty\}}$. Les morphismes de Frobenius relatif sont tous notés $\phi$ afin de simplifier les notations.

\subsection{L'équivalence de Katz}

Soit $\bar{I} \bar{G}_{n, \text { ord }} \rightarrow \bar{X}_{\text {ord }}$ l'espace de modules des trivialisations de $H_{n}^{D}$. C'est un revêtement étale de $\bar{X}_{\text {ord }}$ de groupe $\left(\mathbb{Z} / p^{n} \mathbb{Z}\right)^{\times}$. Soit $\overline{I G}_{\infty, \text { ord }}=\lim _{n} I \bar{G}_{n \text {,ord }}$ le schéma obtenu en passant à la limite projective. Soit $\bar{x}$ un point géométrique de $\bar{X}_{\text {ord }}$ et $\rho: \Pi_{1}\left(\bar{X}_{\text {ord }}, \bar{x}\right) \rightarrow \mathbb{Z}_{p}^{\times}$la représentation de monodromie sur $\left.\overline{I G}_{\infty, \text { ord }}\right|_{\bar{x}}$. Pour tout caractère $\bar{\kappa}_{\chi}: \mathbb{Z}_{p}^{\times} \rightarrow \mathbb{F}_{p}[[T]]^{\times}$, on possède, d'après [21], un $\phi$-module étale $\mathfrak{w}^{\bar{\kappa}_{\chi}}$ associé à $\bar{\kappa}_{\chi} \circ \rho$ dont voici la construction.

Pour tout $n \in \mathbb{Z}_{\geq 0} \cup\{\infty\}$, soit $\mathfrak{I G}_{n, \text { ord },\{\infty\}}$ le produit fibré : $\overline{I G}_{n, \text { ord }} \times_{\text {Spec } \mathbb{F}_{p}} \mathfrak{W}_{\{\infty\}}^{0}$. On

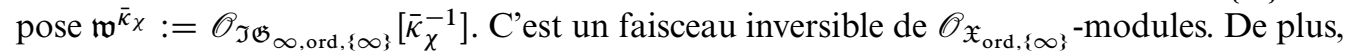
le Frobenius relatif de $\mathfrak{I G}_{\infty, \text { ord },\{\infty\}}$ induit un isomorphisme $\phi^{\star} \mathfrak{w}^{\bar{\kappa}_{\chi}} \simeq \mathfrak{w}^{\bar{\kappa}_{\chi}}$.

\subsection{Tour d'Igusa surconvergente}

Soit $\mathfrak{I G}_{n, r,\{\infty\}} \rightarrow \mathfrak{X}_{r,\{\infty\}}$ la tour d'Igusa partielle. On a des morphismes de transition finis : $\mathfrak{I G}_{n, r,\{\infty\}} \rightarrow \mathfrak{I G}_{n-1, r,\{\infty\}}$. Soit $\mathfrak{I G}_{\infty, r,\{\infty\}}$ la limite projective de ces morphismes dans la catégorie des schémas formels $T$-adiques. Nous allons donner une description simple et une interprétation modulaire de ces tours d'Igusa.

Soit $\bar{I} \bar{G}_{n}$ la normalisation de $\bar{X}$ dans $\bar{I} \bar{G}_{n, \text { ord }}$. La proposition suivante donne une interprétation modulaire de $\bar{I} \bar{G}_{n}$.

Proposition 4.1. - Soit $R$ une $\mathbb{F}_{p}$-algèbre normale. Soit $x \in \bar{X}(R)$ correspondant à un schéma semi-abélien $E / R$ d'invariant de Hasse non nul. Alors $\left.\bar{I}_{n}\right|_{x}(R)$ est l'ensemble des morphismes de schémas en groupes $\mathbb{Z} / p^{n} \mathbb{Z} \rightarrow H_{n}^{D}$ qui sont des isomorphismes au-dessus de Spec $R[1 / \mathrm{Ha}]$.

Démonstration. - C'est évident.

Soit alors $\overline{\mathfrak{I G}}_{n, r}$ le produit fibré : $\overline{I G}_{n} \times_{\bar{X}} \mathfrak{X}_{r,\{\infty\}}$.

LEMme 4.1. - On possède un isomorphisme canonique $\overline{\mathfrak{I G}}_{n, r} \simeq \mathfrak{I G}_{n, r,\{\infty\}}$.

Démonstration. - Les schémas formels $\overline{\mathfrak{I G}}_{n, r}$ et $\mathfrak{I G}_{n, r,\{\infty\}}$ sont finis sur $\mathfrak{X}_{r,\{\infty\}}$ et ils ont clairement la même fibre générique $\mathscr{T} \mathscr{G}_{n, r,\{\infty\}}$. Il suffit donc de voir que $\mathfrak{I} \mathfrak{G}_{n, r}$ est normale. Si Spec $A$ est un ouvert affine de $\bar{X}$, Spec $A_{n}$ l'ouvert affine de $\bar{I} \bar{G}_{n}$ au-dessus, alors le tube de Spec $A$ dans $\mathfrak{I G}_{n, r}$ est $\operatorname{Spf} A_{n}[[T]]\left\langle T / \mathrm{Ha}^{p^{r+1}}\right\rangle$. L'anneau $A_{n}$ est régulier et le morphisme $A_{n} \rightarrow A_{n}[[T]]\left\langle T / \mathrm{Ha}^{p^{r+1}}\right\rangle$ est régulier, donc $A_{n}[[T]]\left\langle T / \mathrm{Ha}^{p^{r+1}}\right\rangle$ est régulier et donc normal.

On déduit alors la proposition suivante :

Proposition 4.2. - 1. Pour toute $\mathbb{F}_{p}[[T]]$-algèbre $R$ normale, plate, $T$-adiquement complète, $\mathfrak{I G}_{n, r,\{\infty\}}(R)$ est l'ensemble des classes d'isomorphisme de triplets $\left(x, \eta, \psi_{n}\right)$ où $x \in \bar{X}(R)$ correspond à un schéma semi-abélien $E / R, \eta \in \omega_{E}^{-(p-1)} p^{r+1}$ vérifie $\mathrm{Ha}^{p^{r+1}} \eta=T$ et $\psi_{n}: \mathbb{Z} / p^{n} \mathbb{Z} \rightarrow H_{n}^{D}$ est un morphisme qui est un isomorphisme au-dessus de Spec $R[1 / \mathrm{Ha}]$. 
2. Pour tout $\mathbb{F}_{p}[[T]]$-algèbre $R$ normale, plate, $T$-adiquement complète, $\mathfrak{I} \mathfrak{G}_{\infty, r,\{\infty\}}(R)$ est l'ensemble des classes d'isomorphismes de triplets $(x, \eta, \psi)$ où $(x, \eta)$ sont comme audessus et $\psi: \mathbb{Q}_{p} / \mathbb{Z}_{p} \simeq$ colim $H_{n}^{D}$ est un morphisme qui est un ismomorphisme au-dessus de $\operatorname{Spec} R[1 / \mathrm{Ha}]$.

\subsection{Surconvergence de l'équivalence de Katz}

Dans ce numéro, nous allons montrer que la construction de Katz garde un sens dans un voisinage du lieu ordinaire et qu'elle fournit les bons objets.

4.4.1. Le faisceau $\mathfrak{w}_{\{\infty\}}$. - Le but de ce paragraphe est de montrer que le faisceau $\mathfrak{w}^{\bar{\kappa}_{\chi}}$ peut se réaliser sur $\mathfrak{X}_{r,\{\infty\}}$ au lieu de $\mathfrak{X}_{\text {ord },\{\infty\}}$. Commençons par un lemme sur la ramification du morphisme $h_{n+1}: \bar{I} G_{n+1} \rightarrow \bar{I} G_{\mathrm{n}}$ (avec la convention $\overline{I G}_{0}=\bar{X}$ ). Notons $\operatorname{Tr}_{I G}:\left(h_{n+1}\right)_{\star} \mathscr{O}_{I \bar{G}_{n+1}} \rightarrow \mathscr{O}_{I G_{\mathrm{n}}}$ la trace de ce morphisme. La source et le but du morphisme $\operatorname{Tr}_{\text {IG }}$ dépendent de $n$, mais on l'omet de la notation pour simplifier.

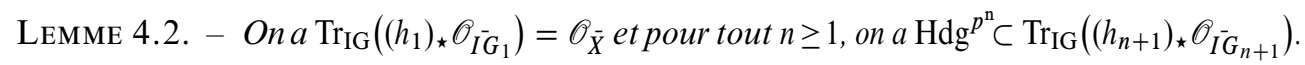

Démonstration. - C'est analogue (en plus simple) à la démonstration de la proposition 3.5 .

Corollaire 4.1. - Soit Spec A un ouvert de $\bar{X}$ sur lequel le faisceau Hdg est trivial. Identifions l'invariant de Hasse Ha avec un générateur de ce faisceau. Alors il existe une suite d'éléments $c_{0}=1, c_{n} \in \mathrm{Ha}^{-\frac{p^{\mathrm{n}}-p}{p-1}} \mathscr{O}_{\overline{I G}_{n}}(\operatorname{Spec} A)$ pour $n \geq 1$ tels que $\operatorname{Tr}_{\mathrm{IG}}\left(c_{n}\right)=c_{n-1}$.

Définissons alors $\mathfrak{w}_{\{\infty\}}$ comme le sous-faisceau de $\mathscr{O}_{\mathfrak{I} \mathfrak{G}_{\infty, r,\{\infty\}}}$ des fonctions $\bar{\kappa}^{-1}$-variantes pour l'action de $\mathbb{Z}_{p}^{\times}$.

ThÉorème 4.1. - Le faisceau $\mathfrak{w}_{\{\infty\}}$ est un faisceau inversible sur $\mathfrak{X}_{r,\{\infty\}}$ pour $r \geq 2$ (resp. $r \geq 3$ si $p=2$ ).

Démonstration. - On peut travailler localement pour la topologie de Zariski sur $\mathfrak{X}_{r,\{\infty\}}$. Soit donc Spec $A$ un ouvert de $\bar{X}$, supposons $\omega_{E}$ libre sur Spec $A$ et identifions l'invariant de Hasse Ha avec un scalaire. Soit Spec $A_{n}$ l'image inverse de Spec $A$ dans $I^{-} G_{n}$, Spf $A_{n}[[T]]\left\langle T / \mathrm{Ha}^{p^{r+1}}\right\rangle$ l'ouvert correspondant dans $\mathfrak{I G}_{n, r,\{\infty\}}$ et $\operatorname{Spf} A_{\infty}[[T]]\left\langle T / \mathrm{Ha}^{p^{r+1}}\right\rangle$ l'ouvert correspondant dans $\mathfrak{I G}_{\infty, r,\{\infty\}}$. On a noté $A_{\infty}=\operatorname{colim}_{n} A_{n}$.

Comme $\left(A_{n}\right)^{\left(\mathbb{Z} / p^{n} \mathbb{Z}\right)^{\times}}=A,\left(A_{\infty}\right)^{\mathbb{Z}_{p}^{\times}}=A$ et donc $\left(A_{\infty}[[T]]\left\langle T / \mathrm{Ha}^{p^{k}}\right\rangle\right)^{\left(\mathbb{Z}_{p}\right)^{\times}}=A[[T]]\left\langle T / \mathrm{Ha}^{p^{r+1}}\right\rangle$. Pour démontrer le théorème, il suffit donc de trouver un élément inversible $x \in A_{\infty}[[T]]\left\langle T / \mathrm{Ha}^{p^{r+1}}\right\rangle$ tel que $\sigma(x)=\bar{\kappa}^{-1}(\sigma) . x$ pour tout $\sigma \in \mathbb{Z}_{p}^{\times}$.

D'après le corollaire 4.1, il existe des éléments $c_{0}=1, c_{1}=\frac{1}{p-1}, c_{n} \in \mathrm{Ha}^{-\frac{p^{\mathrm{n}}-p}{p-1}} A_{n}$ vérifiant $\operatorname{Tr}_{A_{n} / A_{n-1}}\left(c_{n}\right)=c_{n-1}$.

Définissons alors $b_{0}=1$ et $b_{n}=\sum_{\sigma \in\left(\mathbb{Z} / p^{n} \mathbb{Z}\right)^{\times}} \bar{\kappa}(\tilde{\sigma}) \sigma\left(c_{n}\right) \in \mathrm{Ha}^{-\frac{p^{n}-p}{p-1}} A_{n}[[T]]$ pour tout $n \geq 1$, où $\tilde{\sigma} \in \mathbb{Z}_{p}^{\times}$est un relèvement de $\sigma \in\left(\mathbb{Z} / p^{n} \mathbb{Z}\right)^{\times}$. Soit $\tilde{\sigma}^{\prime}$ un autre choix de relèvement de $\sigma$ donnant un autre élément $b_{n}^{\prime}$. Grace au lemme 2.3, on voit facilement que :

$$
b_{n}=b_{n}^{\prime} \bmod T^{p^{n-1}} \mathrm{Ha}^{-\frac{p^{n}-p}{p-1}} \quad \text { si } n \geq 1 \text { et } p \geq 3,
$$




$$
\begin{array}{ll}
b_{n}=b_{n}^{\prime} \bmod T^{p^{n-2}} \mathrm{Ha}^{-\frac{p^{n}-p}{p-1}} & \text { si } n \geq 2 \text { et } p=2, \\
b_{n}=b_{n}^{\prime} \bmod T & \text { si } n=1 \text { et } p \geq 3 .
\end{array}
$$

On vérifie également que :

$$
\begin{array}{rlrl}
b_{n}-b_{n-1} & \in T^{p^{n-2} \mathrm{Ha}^{-\frac{p^{n}-p}{p-1}} A_{n}[[T]]} \quad & \text { si } n \geq 2 \text { et } p \geq 3 \text { ou } n=2 \text { et } p=2, \\
b_{n}-b_{n-1} & \in T^{p^{n-3}} \mathrm{Ha}^{-\frac{p^{n}-p}{p-1}} A_{n}[[T]] & \text { si } n \geq 3 \text { et } p=2, \\
b_{1}-1 & \in T A[[T]] . & &
\end{array}
$$

Indiquons la démonstration dans le cas $n \geq 2$ et $p \neq 2$, les autres cas sont analogues. On a

$$
\begin{aligned}
b_{n} & =\sum_{\sigma \in\left(\mathbb{Z} / p^{n} \mathbb{Z}\right)^{\times}} \bar{\kappa}(\tilde{\sigma}) \sigma \cdot c_{n} \\
& =\sum_{\tau \in\left(\mathbb{Z} / p^{n-1} \mathbb{Z}\right)^{\times}} \bar{\kappa}(\tilde{\tau}) \cdot \tilde{\tau}\left(\sum_{\sigma \in 1+p^{n-1} \mathbb{Z} / p^{n} \mathbb{Z}} \bar{\kappa}(\tilde{\sigma}) \sigma \cdot c_{n}\right) \\
& =\sum_{\tau \in\left(\mathbb{Z} / p^{n-1} \mathbb{Z}\right)^{\times}} \bar{\kappa}(\tilde{\tau}) \cdot \tilde{\tau}\left(c_{n-1}+\sum_{\sigma \in 1+p^{n-1} \mathbb{Z} / p^{n} \mathbb{Z}}(\bar{\kappa}(\tilde{\sigma})-1) \sigma \cdot c_{n}\right) .
\end{aligned}
$$

Et donc

$$
b_{n}-b_{n-1}=\sum_{\tau \in\left(\mathbb{Z} / p^{n-1} \mathbb{Z}\right)^{\times}} \bar{\kappa}(\tilde{\tau}) \tau \cdot c_{n-1}-b_{n-1}+\sum_{\tau \in\left(\mathbb{Z} / p^{n-1} \mathbb{Z}\right)^{\times}} \bar{\kappa}(\tilde{\tau}) \cdot \tilde{\tau}\left(\sum_{\sigma \in 1+p^{n-1} \mathbb{Z} / p^{n} \mathbb{Z}}(\bar{\kappa}(\tilde{\sigma})-1) \sigma \cdot c_{n}\right) \text {. }
$$

Si $\sigma \in 1+p^{n-1} \mathbb{Z} / p^{n} \mathbb{Z}$, alors $\bar{\kappa}(\tilde{\sigma})-1 \in T^{p^{n-2}} \mathbb{F}_{p}[[T]]$ et donc,

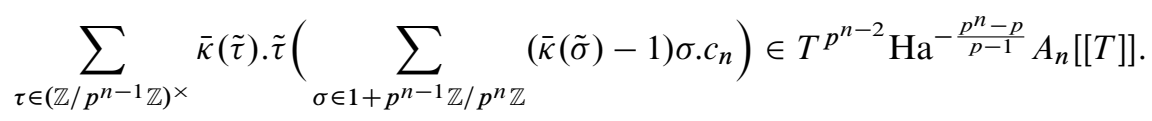

D'autre part, $\sum_{\tau \in\left(\mathbb{Z} / p^{n-1} \mathbb{Z}\right)^{\times}} \bar{\kappa}(\tilde{\tau}) \tau . c_{n-1}-b_{n-1} \in T^{p^{n-2}} \mathrm{Ha}^{-\frac{p^{n-1}-p}{p-1}} A_{n}[[T]]$.

Par récurrence, on vérifie ensuite que $b_{n} \in A_{\infty}\left[\left[T / \mathrm{Ha}^{p^{2}}\right]\right]$ (resp. $A_{\infty}\left[\left[T / \mathrm{Ha}^{p^{3}}\right]\right]$ si $p=2$ ) et que la suite $b_{n}$ converge vers un élément $b_{\infty}$ pour la topologie $\left(T / \mathrm{Ha}^{p^{2}}\right)$-adique (resp. $\left(T / \mathrm{Ha}^{p^{3}}\right)$-adique si $\left.p=2\right)$. On a une inclusion continue $A_{\infty}\left[\left[T / \mathrm{Ha}^{p^{2}}\right]\right] \subset A_{\infty}[[T]]\left\langle T / \mathrm{Ha}^{p^{3}}\right\rangle$ car

$$
\frac{T^{p^{n-2}}}{\mathrm{Ha}^{p^{\mathrm{n}}}}=T^{p^{n-3}(p-1)}\left(\frac{T}{\mathrm{Ha}^{p^{3}}}\right)^{p^{n-3}} .
$$

Il en résulte que $b_{\infty} \in A_{\infty}[[T]]\left\langle T / \mathrm{Ha}^{p^{3}}\right\rangle$ (resp. $b_{\infty} \in A_{\infty}[[T]]\left\langle T / \mathrm{Ha}^{p^{4}}\right\rangle$ si $p=2$ ). Par construction, $\sigma \cdot b_{\infty}=\bar{\kappa}^{-1}(\sigma) \cdot b_{\infty}$. Enfin, $b_{\infty}-1 \in \frac{T}{\mathrm{Ha}^{p^{3}}} A_{\infty}[[T]]\left\langle T / \mathrm{Ha}^{p^{3}}\right\rangle$ (resp. $b_{\infty}-1 \in \frac{T}{\mathrm{Ha}^{p^{4}}} A_{\infty}[[T]]\left\langle T / \mathrm{Ha}^{p^{4}}\right\rangle$ si $p=2$. Comme $\frac{T}{\mathrm{Ha}^{p^{3}}}$ (resp. $\frac{T}{\mathrm{Ha}^{p^{4}}}$ si $p=2$ ) est topologiquement nilpotent, il en résulte que $b_{\infty}$ est inversible.

4.4.2. Le faisceau des formes de poids $\bar{\kappa}_{\chi}$. - Soit $\chi: \mathbb{Z} / q \mathbb{Z}^{\times} \rightarrow O_{\mathbb{C}_{p}}^{\times}$un caractère et $\bar{\kappa}_{\chi}: \mathbb{Z}_{p}^{\times} \rightarrow \mathbb{F}_{p}[[T]]^{\times}$le caractère qui envoie $\exp (q)$ sur $1+T$ et qui vaut la réduction modulo $\mathfrak{m}_{\mathscr{C}_{\mathbb{C}}}$ de $\bar{\chi}$ de $\chi$ sur $\mathbb{Z} / q \mathbb{Z}^{\times}$. Supposons $p \neq 2$ pour un instant. Soit $h_{1}: \bar{I}_{1} \rightarrow X$. Soit $\bar{\omega} \bar{\chi}:=\left(h_{1}\right)_{\star} \mathcal{O}_{I G_{1}}\left[\bar{\chi}^{-1}\right]$ le faisceau cohérent ainsi défini sur $\bar{X}$.

Lemme 4.3. - Le faisceau $\bar{\omega} \bar{x}$ est inversible. 
Démonstration. - Le faisceau $\bar{\omega}^{\bar{\chi}}$ est cohérent et sans torsion donc projectif. On calcule son rang sur le lieu ordinaire, et il vaut évidemment 1.

Si $p=2$, alors le caractère résiduel $\bar{\chi}$ est trivial et $\bar{\omega}^{\bar{\chi}}$ est, par définition, le faisceau trivial. On note $\mathfrak{w}^{\bar{\chi}}$ l'image inverse de $\bar{\omega}^{\bar{\chi}}$ sur $\mathfrak{X}_{r,\{\infty\}}$ pour tout $r$. On pose alors $\mathfrak{w}_{r}^{\bar{\kappa}_{\chi}}=\mathfrak{w}_{\{\infty\}} \otimes \mathfrak{w}^{\bar{\chi}}$.

4.4.3. Fonctorialité à la restriction. - Soit $r^{\prime} \geq r \geq 2(\operatorname{resp} r \geq 3$ si $p=2)$. On a un diagramme commutatif :

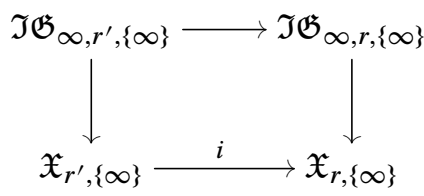

qui induit des immersions ouvertes sur la fibre générique. Le morphisme $\mathfrak{I G}_{\infty, r^{\prime},\{\infty\}} \rightarrow$ $\mathfrak{I G}_{\infty, r,\{\infty\}}$ est équivariant sous l'action du groupe $\mathbb{Z}_{p}^{\times}$. Par conséquent, on dispose d'un morphisme canonique $i^{\star} \mathfrak{w}_{r}^{\bar{\kappa}_{\chi}} \rightarrow \mathfrak{w}_{r^{\prime}}^{\bar{\kappa}_{\chi}}$.

Proposition 4.3. - Le morphisme précédent est un isomorphisme.

Démonstration. - Tensorisons le morphisme $i^{\star} \mathfrak{w}_{r}^{\bar{\kappa}_{\chi}} \rightarrow \mathfrak{w}_{r^{\prime}}^{\bar{\kappa}_{\chi}} \operatorname{par}\left(i^{\star} \mathfrak{w}_{\mathrm{r}}{ }^{\bar{\kappa}_{\chi}}\right)^{-1}$. Le faisceau $\left(i^{\star} \mathfrak{w}_{r}^{\bar{\kappa}_{\chi}}\right)^{-1} \otimes \mathfrak{w}_{r^{\prime}}^{\bar{\kappa}_{\chi}}$ s'identifie au sous-faisceau de $\mathscr{O}_{\mathfrak{I} \mathfrak{G}_{\infty, r^{\prime},\{\infty\}}}$ des sections invariantes sous $\mathbb{Z}_{p}^{\times}$, lequel est égal à $\mathscr{O}_{\mathfrak{X}_{r^{\prime},\{\infty\}}}$.

Dorénavant, on note $\mathfrak{w}^{\bar{\kappa}_{\chi}}$ au lieu de $\mathfrak{w}_{r}^{\bar{\kappa}_{\chi}}$.

4.4.4. Fonctorialité pour le Frobenius. - Soit $E$ le schéma semi-abélien universel sur $\bar{X}$. On dispose aussi d'un morphisme de Frobenius $F: E \rightarrow E / H_{1}=E^{(p)}$. Ce morphisme induit des injections $F^{D}: H_{n}\left(E^{\prime}\right)^{D} \rightarrow H_{n+1}(E)^{D}$ puis un morphisme injectif $F^{D}: \operatorname{colim}_{n} H_{n}\left(E^{\prime}\right)^{D} \rightarrow \operatorname{colim}_{n} H_{\mathrm{n}}(E)^{D}$ qui est un isomorphisme sur le lieu ordinaire. On en déduit une application : $\phi: \mathfrak{X}_{r+1,\{\infty\}} \rightarrow \mathfrak{X}_{r,\{\infty\}}$ que l'on peut étendre en une application $\phi: \mathfrak{I G}_{\infty, r+1,\{\infty\}} \rightarrow \mathfrak{I G}_{\infty, r,\{\infty\}}$, donnée par la règle suivante : A tout morphisme $\psi: \mathbb{Q}_{p} / \mathbb{Z}_{p} \rightarrow \operatorname{colim}_{n} H_{n}(E)^{D}$, on associe le morphisme $\psi^{\prime}$ défini sur le lieu ordinaire par $\left(F^{D}\right)^{-1} \circ \psi: \mathbb{Q}_{p} / \mathbb{Z}_{p} \rightarrow \operatorname{colim}_{n} H_{n}\left(E^{\prime}\right)^{D}$ et qui se prolonge partout par normalité. Si $i: \mathfrak{X}_{r+1,\{\infty\}} \rightarrow \mathfrak{X}_{r,\{\infty\}}$ désigne le morphisme de la section précédente, alors on a un morphisme canonique $i^{\star} \mathfrak{w}^{\bar{\kappa}_{\chi}} \rightarrow \phi^{\star} \mathfrak{w}^{\bar{\kappa}_{\chi}}$.

Proposition 4.4. - Le morphisme $i^{\star} \mathfrak{w}^{\bar{\kappa}_{\chi}} \rightarrow \phi^{\star} \mathfrak{w}^{\bar{\kappa}_{\chi}}$ est un isomorphisme.

Démonstration. - Similaire à la démonstration précédente.

4.4.5. Définitions. - On peut maintenant définir les espaces de formes surconvergentes.

DÉFINITION 4.1. - Une forme $r$-surconvergente entière de poids $\bar{\kappa}_{\chi}$ est une section globale du faisceau inversible $\mathfrak{w}^{\bar{\kappa}_{\chi}}$.

On note $\omega^{\bar{\kappa}_{\chi}}$ le faisceau inversible sur ${ }^{\mathscr{C}} \mathcal{C}_{r,\{\infty\}}$ fibre générique de $\mathfrak{w}^{\bar{\kappa}_{\chi}}$. Une forme surcon-

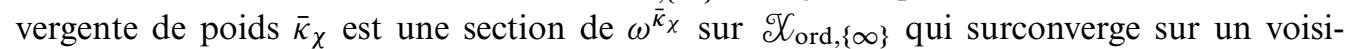
nage $\mathscr{C}_{r,\{\infty\}}$. On note $M_{\bar{\kappa}_{\chi}}^{\dagger}$ le $\mathbb{F}_{p}((T))$-espace des formes surconvergentes de poids $\bar{\kappa}_{\chi}$. C'est une limite inductive compact d'espaces de Banach. 
4.4.6. Description modulaire. - Une forme $r$-surconvergente entière $f$ de poids $\bar{\kappa}_{\chi}$ est une loi fonctorielle qui à

1. une $\mathbb{F}_{p}[[T]]$-algèbre $T$-adiquement complète, normale, plate $R$,

2. $x \in X(R)$ correspondant à un schéma semi-abélien $E / R$ et une structure de niveau $N$,

3. $\eta \in \omega_{E}^{-(p-1) p^{r+1}}$ qui vérifie $\mathrm{Ha}^{p^{r+1}} \eta=T$,

4. une trivialisation $\psi: \mathbb{Q}_{p} /\left.\mathbb{Z}_{p} \simeq \operatorname{colim}_{n} H_{n}^{D}\right|_{R[1 / \mathrm{Ha}]}$,

associe un élément de $f(x, \eta, \psi) \in R$ tel que $f(x, \eta, \sigma . \psi)=\bar{\kappa}_{\chi}^{-1}(\sigma) f(x, \eta, \psi)$ pour tout $\sigma \in \mathbb{Z}_{p}^{\times}$.

4.4.7. Opérateur $U_{p}$. - On verra dans la proposition 6.2 qu'on peut définir un morphisme $p^{-1} \operatorname{Tr}_{\phi}: \phi_{\star} \mathscr{O}_{\mathscr{S}}{ }_{r+1,\{\infty\}} \rightarrow \mathscr{O}_{\mathscr{E}}{ }_{r,\{\infty\}}$. On définit alors l'opérateur $U_{p}$ par la règle :

$$
U_{p}: \mathrm{H}^{0}\left(\mathscr{C}_{\mathrm{r}}, \omega^{\bar{\kappa}_{\chi}}\right) \rightarrow \mathrm{H}^{0}\left(\mathscr{\mathscr { C }}_{r+1}, i^{\star} \omega^{\bar{\kappa}_{\chi}}\right) \simeq \mathrm{H}^{0}\left(\mathscr{\mathscr { C }}_{r+1}, \phi^{\star} \omega^{\bar{\kappa}_{\chi}}\right) \stackrel{p^{-1} \operatorname{Tr}_{\phi}}{\longrightarrow} \mathrm{H}^{0}\left(\mathscr{C}_{\mathrm{r}}, \omega^{\bar{\kappa}_{\chi}}\right) .
$$

Cet opérateur est compact et il possède donc une série caractéristique.

4.4.8. Action du reste de l'algèbre de Hecke. - L'action de l'algèbre de Hecke hors $p$ peut être définie sans problème, de façon géométrique, comme dans le cas des espaces de formes modulaires classiques.

\section{Intégralité de la famille universelle de faisceaux sur l'espace $\mathscr{W}_{[0, \infty[}$}

L'objectif de cette section est de construire une famille de faisceaux modulaires sur l'espace adique $\mathscr{W}_{[0, \infty[}$, ainsi que des modèles entiers. La famille de faisceaux sur $\mathscr{W}_{[0, \infty[}$ a été construite dans [3] et [25]. La nouveauté ici est que nous construisons des modèles entiers inversibles canoniques pour ces faisceaux .

\subsection{Résultats principaux}

On renvoie au numéro 3.3 pour la définition des objets considérés. Dans cette partie, $I=[0,1]$ ou $\left[p^{k}, p^{k^{\prime}}\right]$ pour $k, k^{\prime} \in \mathbb{Z}_{\geq 0}$. Si $I=[0,1]$, on pose $k=k^{\prime}=0$ pour avoir des notations uniformes.

5.1.1. En géométrie formelle. - Le résultat principal de cette partie est le suivant. Sa démonstration fait l'objet des numéros 5.2, 5.3 et 5.4.

THÉORÈme 5.1. - Supposons $r \geq 1$ et $r+k \geq k^{\prime}+2$ (resp. $r \geq 2$ et $r+k \geq k^{\prime}+4$ si $p=2$ ). Alors on possède un faisceau inversible $\mathfrak{w}_{I}$ sur $\mathfrak{X}_{r, I}$. Ce faisceau jouit des propriétés suivantes :

1. Soit $\mathfrak{C}_{r, I}$ l'espace adique sur $\mathbb{Q}_{p}$ fibre générique de $\mathfrak{X}_{r, I}$. Le faisceau fibre générique de $\mathfrak{w}_{I}$ est le faisceau des familles de formes surconvergentes sur $\mathscr{C}_{r, I}$ construit dans [3] et [25].

2. On a un opérateur de Frobenius :

$$
i^{\star} \mathfrak{w}_{I} \simeq \phi^{\star} \mathfrak{w}_{I}
$$

où $i: \mathfrak{X}_{r+1, I} \rightarrow \mathfrak{X}_{r, I}$ est le morphisme d'inclusion et $\phi: \mathfrak{X}_{r+1, I} \rightarrow \mathfrak{X}_{r, I}$ est le Frobenius.

3. La construction est fonctorielle en l'intervalle $I$ : si $I^{\prime} \subset I$, et si $i_{I^{\prime}, I}: \mathfrak{X}_{r, I^{\prime}} \rightarrow \mathfrak{X}_{r, I}$ est le morphisme naturel alors $i_{I^{\prime}, I}^{\star} \mathfrak{w}_{I}=\mathfrak{w}_{I^{\prime}}$. 
5.1.2. En géométrie analytique. - Le théorème suivant est essentiellement la traduction analytique du théorème 5.1. On renvoie toujours au numéro 3.3 pour les définitions des objets intervenant ci-dessous.

Théorème 5.2. - Pour tout $r \geq 3$ (resp. $r \geq 5$ si $p=2$ ), on possède un faisceau inversible $\omega_{[0, \infty[}^{\kappa}$ sur l'espace $\mathcal{M}_{r,[0, \infty[}$, ainsi qu'un sous-faisceau $\omega_{[0, \infty[}^{\kappa,+} d e \mathscr{O}_{\mathcal{M}_{r,[0, \infty[}^{+}}^{-}$modules. Les propriétés suivantes sont vérifiées :

1. La restriction du sous-faisceau $\omega_{[0, \infty[}^{\kappa,+}$ à la composante libre $\mathscr{W}_{[0, \infty[}^{0}$ est un faisceau inversible de $\mathscr{O}_{\mathscr{C}_{r,[0, \infty[}^{+}}^{+}$-module.

2. Pour tout caractère localement algébrique $\chi \cdot k: \mathbb{Z}_{p}^{\times} \rightarrow \mathbb{C}_{p}^{\times}$avec $\chi$ un caractère fini et $k \in \mathbb{Z}$ identifié à un point $\kappa$ de $\mathscr{W}^{\mathrm{rig}}, \omega_{\left[0, \infty\left[\left.\right|_{\{\chi . k\}} ^{\kappa}\right.\right.}^{\kappa}=\omega^{\mathrm{k}}(\chi)$ est le faisceau usuel des formes modulaires de poids $k$ et nebentypus $\chi$.

3. Le morphisme de Frobenius $\phi: M_{r+1} \rightarrow M_{r}$ et l'inclusion $i: M_{r+1} \rightarrow M_{r}$ induisent des isomorphismes $\phi^{\star} \omega_{[0, \infty[}^{\kappa} \simeq i^{\star} \omega_{[0, \infty[}^{\kappa}$. Si on se restreint à $\mathcal{W}_{[0, \infty[}^{0}$, on a même des isomorphismes au niveau entier $\phi^{\star} \omega_{[0, \infty[}^{\kappa,+} \simeq i^{\star} \omega_{[0, \infty[}^{\kappa,+}$.

Remarque 5.1. - Pour tout caractère localement algébrique $\chi \cdot k: \mathbb{Z}_{p}^{\times} \rightarrow \mathbb{C}_{p}^{\times}$, on observe que le faisceau classique $\omega^{k}(\chi)$ est bien défini sur $\left.\mathcal{M}_{\mathrm{r}}\right|_{\chi \cdot k}$. Soit $p^{n}$ le conducteur de $\chi$. On vérifie qu'on possède sur $\left.M_{\mathbf{r}}\right|_{\chi \cdot k}$ un sous-groupe canonique d'échelon $n$. En effet, plus le conducteur de $\chi$ est grand, plus le point $\chi \cdot k$ est proche du bord dans $\mathscr{W}$, et donc plus le voisinage strict $\left.\mathcal{M}_{\mathrm{r}}\right|_{\chi \cdot k}$ vu pour la topologie $p$-adique est petit. Ainsi, $\left.\mathcal{M}_{\mathrm{r}}\right|_{\chi \cdot k}$ s'identifie à un ouvert de la courbe modulaire analytique de niveau $\Gamma_{0}\left(p^{n}\right)$ en $p$, où le faisceau $\omega^{k}(\chi)$ est bien défini.

Démonstration. - Expliquons pour commencer comment se ramener à travailler sur la composante libre $\mathscr{W}_{[0, \infty[}^{0}$. On possède un revêtement (tour d'Igusa partielle) $f: \mathscr{J}_{\mathcal{G}_{1, r}} \rightarrow \mathscr{C}_{r}$ qui paramètre les trivialisations du sous-groupe canonique dual $H_{1}^{D}$ (pour $p=2$, on prend $f: \mathscr{J}_{\mathcal{G}_{2, r}} \rightarrow \mathscr{C}_{r}$, qui paramètre les trivialisations de $H_{2}^{D}$ ). C'est un torseur étale sous le groupe $(\mathbb{Z} / q \mathbb{Z})^{\times}$. Pour chaque caractère $\chi:(\mathbb{Z} / q \mathbb{Z})^{\times} \rightarrow \mathbb{Z}_{p}^{\times}$, on peut considérer le faisceau $\left(\omega^{\chi}, \omega^{\chi,+}\right)=\left(f_{\star} \mathscr{O}_{\mathscr{J} \mathscr{G}_{1}}\left[\chi^{-1}\right], f_{\star} \mathscr{O}_{\mathscr{G} \mathscr{G}_{1}}^{+}\left[\chi^{-1}\right]\right)$ où $\left[\chi^{-1}\right]$ désigne le sous-faisceau des sections qui se transforment selon le caractère $\chi^{-1}$. Comme $f$ est un torseur étale, $f_{\star} \mathscr{O}_{\mathscr{g} \mathscr{G}_{1}}\left[\chi^{-1}\right]$ est un faisceau inversible.

Supposons qu'on a construit un faisceau $\left(\omega_{[0, \infty[}^{\kappa}, \omega_{[0, \infty[}^{\kappa,+}\right)$ ayant les propriétés attendues sur $\mathcal{W}_{[0, \infty[}^{0}$. En tensorisant $\left(\omega_{[0, \infty[}^{\kappa}, \omega_{[0, \infty[}^{\kappa,+}\right)$ par les faisceaux $\left(\omega^{\chi}, \omega^{\chi,+}\right)$ où $\chi$ parcourt les caractères du groupe $(\mathbb{Z} / q \mathbb{Z})^{\times}$, on étend la construction sur $\mathscr{W}^{\text {rig }}=\bigsqcup_{\chi} \mathscr{W}_{[0, \infty[}^{0} \cdot \chi$.

L'espace $\mathscr{\omega}_{[0, \infty[}^{0}$ est recouvert par les ouverts $\mathscr{W}_{[0,1]}^{0}$ et $\mathscr{\omega}_{\left[p^{\mathrm{k}}, p^{k+1}\right]}^{0}$ pour $k \geq 0$. Il est donc suffisant de construire les faisceaux sur chacun de ces ouverts et de vérifier qu'ils se recollent.

Soit $I$ un des intervalles $[0,1]$ ou $\left[p^{k}, p^{k+1}\right]$. Considérons le schéma formel $\mathfrak{W}_{I}^{0}=\operatorname{Spf} B_{I}$ et le schéma formel $\mathfrak{X}_{r, I} \rightarrow \mathfrak{W}_{I}^{0}$ qui a pour fibre générique ${ }^{\mathscr{C}} \mathcal{C}_{r, I}$. Comme $r+k \geq k+1+2$ (resp. $\geq k+1+4$ si $p=2$ ), on possède (thm. 5.1) un faisceau inversible $\mathfrak{w}_{I}$ sur $\mathfrak{X}_{r, I} \rightarrow \mathfrak{W}_{I}^{0}$. Celui-ci induit des faisceaux $\left(\omega_{\mathrm{I}}^{\kappa}, \omega_{\mathrm{I}}^{\kappa,+}\right)$ sur la fibre analytique $\mathscr{E}_{r, I}$. Les différentes fonctorialités nous permettent de recoller ces faisceaux. 
5.1.3. Prolongement à l'infini. - Grâce au théorème 5.2, on dispose au-dessus de $\mathscr{C}_{r,[0, \infty[}$ d'un couple $\left(\omega_{[0, \infty[}^{\kappa}, \omega_{[0, \infty[}^{\kappa,+}\right)$ de faisceaux cohérents inversibles de $\mathscr{O}_{\mathscr{E}} \mathscr{C}_{r,[0, \infty[}$ et $\mathscr{O}_{\mathscr{C}_{r,[0, \infty[}}^{+}$-modules. Notre but ultime est de prolonger ces faisceaux à l'infini. Soit $j: \mathscr{V}_{r,[1, \infty[} \hookrightarrow \mathscr{C}_{r,[1, \infty]}$ l'immersion ouverte. On voudrait donc prolonger le faisceau $\omega_{[1, \infty[}^{\kappa}$ en un faisceau $\omega_{[1, \infty]}^{\kappa}$ sur $\mathscr{C}_{r,[1, \infty]}$. Le théorème 1.6 de [23] affirme qu'une fonction bornée sur un ouvert dense de Zariski d'un espace rigide normal se prolonge à tout l'espace. Nous ignorons si ce résultat est valable dans le cadre adique analytique. Dans tous les cas, il est raisonnable de poser $\omega_{[1, \infty]}^{\kappa,+}=j_{\star} \omega_{[1, \infty[}^{\kappa,+}$ et $\omega_{[1, \infty]}^{\kappa}$ est le faisceau associé à $\omega_{[1, \infty]}^{\kappa,+}[1 / T]$. Pour tout ouvert rationnel $V$, une section de $\omega_{[1, \infty]}^{\kappa}$ sur $V$ est donc une section bornée de $\omega_{[1, \infty[}^{\kappa}$ sur $\mathscr{C}_{r,[1, \infty[} \cap V$. C'est pour cette raison que nous avons cherché une structure entière canonique sur les faisceaux de formes surconvergentes. L'exemple suivant montre cependant que ce genre de prolongement est en général pathologique. On vérifiera néanmoins (essentiellement) dans la section 6 que $j_{\star} \omega_{[1, \infty[}^{\kappa,+}$ est bien localement libre dans notre cas.

Exemple 1. - On pose $\mathcal{S}=\mathscr{W}_{[1, \infty]}^{0}$ et $\mathcal{U}=\mathscr{W}_{[1, \infty[}^{0}$. Soit $j: \mathcal{U} \rightarrow \mathcal{S}$. On va prendre $\mathscr{F}=\mathscr{O}_{\varphi}$, le faisceau trivial. Sur chaque intervalle $\left[p^{k}, p^{k+1}\right]$ on pose $\left.\mathscr{F}^{+}\right|_{\omega_{\left[p^{k}, p^{k+1}\right]}^{0}}=$ $\frac{p^{k+1}}{T^{\frac{1-p^{k+1}}{1-p}}} \mathscr{O}_{\mathscr{W}_{\left[p^{k}, p^{k+1}\right]}^{+}}^{+}$. Le faisceau est bien défini, car sur la couronne $\left|T^{p^{k}}\right|=|p|$, on a :

$$
\left|\frac{p^{k+1}}{T^{\frac{1-p^{k+1}}{1-p}}}\right|=\left|\frac{p^{\mathrm{k}}}{T^{\frac{1-p^{\mathrm{k}}}{1-p}}}\right| .
$$

Soit $\tilde{\mathscr{F}}=\left(j_{\star} \mathscr{F}^{+}\right)[1 / T]$. Nous prétendons que le faisceau $\tilde{\mathscr{F}}$ est le prolongement par 0

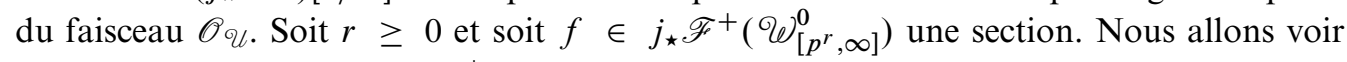
que $f=0$. Clairement, $f \in \mathscr{O}_{\mathcal{W}_{\left[p^{r}, \infty\right]}^{+}}^{0}$. On étend les scalaires de $\mathbb{Z}_{p}$ à une extension finie $\mathcal{O}_{K}$ pour disposer d'un élément $\varpi$ de valuation $1 / p^{r}$. On peut écrire :

$$
f=\sum_{k=0}^{\infty} a_{k} T^{k}+\sum_{l=1}^{\infty} b_{l}\left(\frac{\varpi}{T}\right)^{l} .
$$

Sur chaque couronne $\left|T^{p^{n}}\right|=|p|$, on a par définition $|f| \leq p^{-n+1}$. On obtient alors que :

$$
\begin{aligned}
& \left|a_{k}\right| \leq p^{-n+1+\frac{k}{p^{n}}}, \\
& \left|b_{l}\right| \leq p^{-n+1-\frac{l}{p^{n}+\frac{l}{p^{r}}} .}
\end{aligned}
$$

En faisant tendre $n$ vers $+\infty$, on obtient alors $f=0$.

\subsection{Construction d'un torseur}

Dans cette section, nous construisons un torseur de formes différentielles. Soit $E$ le schéma semi-abélien universel sur $\mathfrak{X}_{r, I}$. On possède un sous-groupe canonique $H_{n}$ d'échelon $n$ pour tout $n \leq r+k$. Soit $\mathfrak{I}_{n, r, I}$ la tour d'Igusa partielle formelle de niveau $n$ et $g_{n}: \mathfrak{I G}_{n, r, I} \rightarrow \mathfrak{X}_{r, I}$ son morphisme structural. Soit $\omega_{\mathrm{E}}$ le faisceau conormal de $E$. Le noyau du morphisme $\omega_{E} \rightarrow \omega_{H_{\mathrm{n}}}$ est $p^{\mathrm{n}} \operatorname{Hdg}^{-\frac{p^{\mathrm{n}}-1}{p-1}} \omega_{\mathrm{E}}$. Sur l'ouvert où $E$ est un schéma abélien, cela résulte du corollaire A.2. Si $E$ est un schéma semi-abélien, ou plus généralement si $E$ est dans l'ouvert ordinaire l'énoncé résulte de l'égalité $\omega_{H_{n}}=\omega_{E\left[p^{n}\right]}$. Ainsi, la 
projection $\omega_{E} \rightarrow \omega_{H_{n}}$ se factorise en un isomorphisme $\omega_{\mathrm{E}} / p^{\mathrm{n}} \mathrm{Hdg}^{-\frac{p^{\mathrm{n}}-1}{p-1}} \simeq \omega_{H_{n}}$. On a alors un diagramme de faisceaux abéliens $f p p f$ sur $\mathfrak{X}_{r, I}$ :

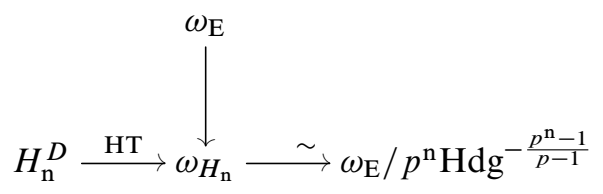

où HT est l'application de Hodge-Tate.

Au-dessus de $\mathfrak{I G}_{n, r, I}$, appelons $P \in H_{n}^{D}$ l'image de 1 par le morphisme universel $\mathbb{Z} / p^{n} \mathbb{Z} \rightarrow H_{n}^{D}$. L'image par HT du point universel $P$ engendre un sous-module $\operatorname{Hdg}^{\frac{1}{p-1}} \omega_{H_{n}}$ de $\omega_{H_{n}}$ d'après la proposition A.3.

On note $f_{n}: \mathfrak{F}_{n, r, I} \rightarrow \mathfrak{I G}_{n, r, I}$ le torseur sous le groupe $1+p^{\mathrm{n}} \operatorname{Hdg}^{-\frac{p^{\mathrm{n}}}{p-1}} \mathbb{G}_{a}$ défini $\operatorname{par} \mathfrak{F}_{n, r, I}(R)=\left\{\omega \in \omega_{\mathrm{E}}, \omega=\mathrm{HT}(P)\right.$ dans $\left.\omega_{\mathrm{E}} / p^{n} \mathrm{Hdg}^{-\frac{p^{\mathrm{n}}-1}{p-1}}\right\}$.

On a une action de $\mathbb{Z}_{p}^{\times}$sur $\mathfrak{F}_{n, r, I}$, donnée par $\lambda(\omega, P)=(\lambda \omega, \lambda P)$. Cette action relève l'action de $\left(\mathbb{Z} / p^{n} \mathbb{Z}\right)^{\times}$sur $\mathfrak{I G}_{n, r, I}$.

Lemme 5.1. - Les actions de $\mathbb{Z}_{p}^{\times}$et $\left(1+p^{n} \mathrm{Hdg}^{-\frac{p^{\mathrm{n}}}{p-1}} \mathbb{G}_{a}\right)$ sur $\mathfrak{F}_{n, r, k}$ proviennent d'une action du groupe $\mathbb{Z}_{p}^{\times}\left(1+p^{n} \mathrm{Hdg}^{-\frac{p^{\mathrm{n}}}{p-1}} \mathbb{G}_{a}\right)$.

Démonstration. - En effet, nous avons $\mathbb{Z}_{p}^{\times} \cap\left(1+p^{n} \operatorname{Hdg}^{-\frac{p^{\mathrm{n}}}{p-1}} \mathbb{G}_{a}\right)=1+p^{n} \mathbb{Z}_{p}$ car $p^{n} \operatorname{Hdg}^{-\frac{p^{\mathrm{n}}}{p-1}} \mathbb{G}_{a} \cap \mathbb{Z}_{p}=p^{n} \mathbb{Z}_{p}$. Mais les actions de $1+p^{n} \mathbb{Z}_{p}$ vu comme sous-groupe de $\mathbb{Z}_{p}^{\times}$ ou de $1+p^{n} \operatorname{Hdg}^{-\frac{p^{\mathrm{n}}}{p-1}} \mathbb{G}_{a}$ coïncident.

\subsection{Le faisceau des familles de formes surconvergentes}

En utilisant le torseur $\mathfrak{F}_{n, r, I}$ nous allons définir le faisceau des familles de formes surconvergentes.

5.3.1. Construction. - On déduit de la proposition 2.1, des accouplements :

$$
\mathfrak{W}_{I}^{0} \times \mathbb{Z}_{p}^{\times}\left(1+p^{k^{\prime}+1} \mathbb{G}_{a}\right) \rightarrow \mathbb{G}_{m} \quad \text { si } p \neq 2
$$

et

$$
\mathfrak{W}_{I}^{0} \times \mathbb{Z}_{p}^{\times}\left(1+p^{k^{\prime}+3} \mathbb{G}_{a}\right) \rightarrow \mathbb{G}_{m} \quad \text { si } p=2 .
$$

Nous supposons à présent que $n \geq k^{\prime}+2$ (resp. $n \geq k^{\prime}+4$ si $p=2$ ). On dispose alors d'un caractère $\kappa: \mathbb{Z}_{p}^{\times}\left(1+p^{\mathrm{n}} \operatorname{Hdg}^{-\frac{p^{\mathrm{n}}}{p-1}} \mathbb{G}_{a}\right) \rightarrow \mathbb{G}_{m}$ de schémas en groupes définis sur $\mathfrak{I}_{n, r, I}$. Il provient de l'accouplement universel car $p^{\mathrm{n}} \mathrm{Hdg}^{-\frac{p^{\mathrm{n}}}{p-1}} \mathbb{G}_{a} \subset p^{k^{\prime}+1} \mathbb{G}_{a}$ (resp. $\subset p^{k^{\prime}+3} \mathbb{G}_{a}$ si $p=2$ ).

Définissons $\mathfrak{w}_{n, r, I}^{1}=\left(f_{n}\right)_{\star} \mathscr{O}_{\mathfrak{F}_{n, r, I}}\left[\kappa^{-1}\right]$, le sous-faisceau de $\left(f_{n}\right)_{\star} \mathscr{O}_{\mathfrak{F}_{n, r, I}}$ des sections qui se transforment selon le caractère $\kappa^{-1}$ sous-l'action de $1+p^{\mathrm{n}} \operatorname{Hdg}^{-\frac{p}{p-1}} \mathbb{G}_{a}$. C'est un faisceau inversible sur $\mathfrak{I G}_{n, r, I}$. Soit $\mathfrak{w}_{n, r, I} \subset\left(g_{n}\right)_{\star} \mathfrak{w}_{n, r, I}^{1}$ le sous-faisceau de $\left(g_{n} \circ f_{n}\right)_{\star} \mathscr{O}_{\mathfrak{F}_{n, r, I}}$ constitué des sections $\kappa^{-1}$-variantes sous l'action du groupe $\mathbb{Z}_{p}^{\times}\left(1+p^{\mathrm{n}} \mathrm{Hdg}^{-\frac{p^{\mathrm{n}}}{p-1}} \mathbb{G}_{a}\right)$. On rappelle que $g_{n}: \mathfrak{I G}_{n, r, I} \rightarrow \mathfrak{X}_{r, I}$ est le morphisme structural. 
5.3.2. Liberté. - Dans ce numéro, nous démontrons l'inversibilité du faisceau $\mathfrak{w}_{n, r, I}$.

Lemme 5.2. - Soit $\left(\mathscr{O}_{\mathfrak{X}_{r, I}}\right)^{00}$ l'idéal des éléments topologiquement nilpotents dans $\mathscr{O}_{\mathfrak{X}_{r, I}}$. Supposons que $r \geq 1$ (resp. $r \geq 2$ si $p=2$ ). Alors $\kappa\left(\mathbb{Z}_{p}^{\times}\right)-1 \subset\left(\mathscr{O}_{\mathfrak{X}_{r, I}}\right)^{00}$ et pour tout $2 \leq l \leq r+k$,

$$
\kappa\left(1+p^{l-1} \mathbb{Z}_{p}\right)-1 \subset \operatorname{Hdg}^{\frac{p^{l}-p}{p-1}}\left(\mathscr{O}_{\mathfrak{X}_{r, I}}\right)^{00}
$$

Démonstration. - Commençons par remarquer que $\kappa\left((\mathbb{Z} / q \mathbb{Z})^{\times}\right)=1$ car on s'est restreint à la composante libre de l'espace des poids. Par conséquent, $\kappa\left(\mathbb{Z}_{p}^{\times}\right)=\kappa\left(1+q \mathbb{Z}_{p}\right)$. Il suffit donc de traiter les cas $l \geq 2$ si $p \neq 2$ et $l \geq 3$ si $p=2$. Traitons d'abord le cas $p \neq 2$. Pour $l \geq 2$, on a, d'après le lemme $2.3, \kappa\left(1+p^{l-1} \mathbb{Z}_{p}\right)-1 \subset\left(T^{p^{l-2}}, p\right) \Lambda$. Comme $p \in \operatorname{Hdg}^{p^{r+k+1}} \mathscr{O}_{\mathfrak{X}_{r, I}}$, pour $l \leq r+k, p \mathrm{Hdg}^{-p^{l}}$ est dans l'idéal des éléments topologiquement nilpotent. Par ailleurs, comme $r \geq 1, T \in \operatorname{Hdg}^{p^{2}} \mathscr{O}_{\mathfrak{X}_{r, I}} \operatorname{donc} T^{p^{l-2}} \in \operatorname{Hdg}^{p^{1}} \mathscr{O}_{\mathfrak{X}_{r, I}}$. Comme $\frac{p^{l}-p}{p-1}<p^{l}$, il en résulte que $T^{p^{l-2}} \mathrm{Hdg}^{-\frac{p^{l}-p}{p-1}}$ est dans l'idéal des éléments topologiquement nilpotent. Pour $p=2$ et $l \geq 3$, on a $\kappa\left(1+p^{l-1} \mathbb{Z}_{p}\right)-1 \subset\left(T^{p^{l-3}}, p\right) \Lambda$. Le raisonnement est identique et utilise seulement $r \geq 2$ au lieu de $r \geq 1$.

Lemme 5.3. - L'inclusion $\mathscr{O}_{\mathfrak{J G}_{n, r, I}} \rightarrow\left(f_{n}\right)_{\star} \mathscr{O}_{\mathfrak{F}_{n, r, I}}$ induit par passage au quotient un isomorphisme :

$$
\mathscr{O}_{\mathfrak{I} \mathfrak{G}_{n, r, I}} / q \rightarrow \mathfrak{w}_{n, r, I}^{1} / q
$$

Démonstration. - Sur tout ouvert affine de $\mathfrak{I G}_{n, r, I}$, on a une section du morphisme $\mathfrak{F}_{n, r, I} \rightarrow \mathfrak{I G}_{n, r, I}$, donnée par le choix d'une forme différentielle $\operatorname{HT(}(P)$ qui relève $\operatorname{HT}(P)$. Remarquons que deux sections diffèrent par un élément du groupe $1+p^{\mathrm{n}} \operatorname{Hdg}^{-\frac{p^{n}}{p-1}} \mathbb{G}_{a}$. On dispose, pour chaque ouvert affine $U$ et pour chaque section $\tilde{H T}(P)$, d'un isomorphisme $\left.\left.\mathfrak{w}_{n, r, I}^{1}\right|_{U} \rightarrow \mathscr{O}_{\mathfrak{I G}} \mathfrak{G}_{n, r, I}\right|_{U}$, donné par l'évaluation en la section. Réduisant modulo $q$ et utilisant le fait que $\kappa\left(1+p^{\mathrm{n}} \mathrm{Hdg}^{-\frac{p^{n}}{p-1}} \mathbb{G}_{a}\right) \subset 1+q \mathbb{G}_{a}$ d'après la proposition 2.1, on remarque que l'ambiguité dans le choix de la section disparaît. On obtient donc un isomorphisme canonique $\mathfrak{w}_{n, r, I}^{1} / q \rightarrow \mathscr{O}_{\mathfrak{I} \mathfrak{G}_{n, r, I}} / q$ qui est l'inverse de l'isomorphisme cherché.

Soit Spf $A$ un ouvert affine de $\mathfrak{X}_{r, I}$. On suppose que Hdg est principal sur $\operatorname{Spf} A$, engendré par un élément Ha. D'après le corollaire 3.1, pour tout $0 \leq n \leq r+k$, il existe des éléments

$$
c_{0}=1, c_{n} \in \tilde{\mathrm{Ha}}{ }^{-\frac{p^{\mathrm{n}}-p}{p-1}} \mathscr{O}_{\mathfrak{I G}_{n, r, I}}(\operatorname{Spf} A) \sin \geq 1
$$

tels que $\operatorname{Tr}_{\mathfrak{I G}}\left(c_{n}\right)=c_{n-1}$ si $n \geq 1$. Définissons un morphisme pour $r+k \geq n \geq k^{\prime}+2$ (resp. $n \geq k^{\prime}+4$ si $\left.p=2\right)$ :

$$
\begin{aligned}
e_{c_{n}}:\left(g_{n}\right)_{\star} \mathfrak{w}_{n, r, I}^{1}(\operatorname{Spf} A) & \rightarrow \tilde{\mathrm{Ha}}{ }^{-\frac{p^{\mathrm{n}}-p}{p-1}} \mathfrak{w}_{n, r, I}(\operatorname{Spf} A) \\
s & \mapsto \sum_{\sigma \in\left(\mathbb{Z} / p^{\mathrm{n}} \mathbb{Z}\right)^{\times}} \kappa(\sigma) \sigma\left(c_{n} s\right) .
\end{aligned}
$$

Lemme 5.4. - Supposons $r \geq 1$ (resp. $r \geq 2$ si $p=2$ ). Soit $s \in\left(g_{n}\right)_{\star} \mathfrak{w}_{n, r, I}^{1}(\operatorname{Spf} A)$ un élément qui vérifie $s=1 \bmod p$ au sens du lemme 5.3. Alors $e_{c_{n}}(s) \in \mathfrak{w}_{n, r, I}(\operatorname{Spf} A)$. De plus, $\mathfrak{w}_{n, r, I}(\operatorname{Spf} A)$ est le A-module libre engendré par $e_{c_{n}}(s)$. 
Démonstration. - On a $s=1+p h$ pour une section $h \in \mathfrak{F}_{n, r, I}(\operatorname{Spf} A)$. Il en résulte que

$$
e_{c_{n}}(s)=\sum_{\sigma \in\left(\mathbb{Z} / p^{\mathrm{n}} \mathbb{Z}\right)^{\times}} \kappa(\tilde{\sigma}) \tilde{\sigma}\left(c_{n}\right)+p \sum_{\sigma \in\left(\mathbb{Z} / p^{\mathrm{n}} \mathbb{Z}\right)^{\times}} \kappa(\tilde{\sigma}) \tilde{\sigma}\left(c_{n} h\right) .
$$

Dans cette formule, $\tilde{\sigma}$ est un relèvement arbitraire de $\sigma$ dans $\mathbb{Z}_{p}^{\times}$. Comme $\tilde{\mathrm{Ha}}^{p+k+1} \mid p$ et $\frac{p^{n}-p}{p-1}<p^{r+k+1}$, il en résulte que $p \sum_{\sigma \in\left(\mathbb{Z} / p^{\mathrm{n}} \mathbb{Z}\right)^{\times}} \kappa(\tilde{\sigma}) \tilde{\sigma}\left(c_{n} h\right) \in A^{00} \mathfrak{F}_{n, r, I}(\operatorname{Spf} A)$ où $A^{00}$ est l'idéal des éléments topologiquement nilpotent dans $A$.

Montrons que

$$
\sum_{\sigma \in\left(\mathbb{Z} / p^{\mathrm{n}} \mathbb{Z}\right)^{\times}} \kappa(\tilde{\sigma}) \tilde{\sigma}\left(c_{n}\right) \in 1+A^{00} \mathfrak{F}_{n, r, I}(\operatorname{Spf} A) .
$$

Nous allons en fait vérifier par récurrence sur $0 \leq l \leq k+r$ que $\sum_{\sigma \in\left(\mathbb{Z} / p^{1} \mathbb{Z}\right)^{\times}} \kappa(\tilde{\sigma}) \tilde{\sigma}\left(c_{l}\right) \in$ $1+A^{00} \mathfrak{F}_{l, r, I}(\operatorname{Spf} A)$ pour n'importe quel relèvement $\tilde{\sigma}$ de $\sigma$. Pour $l=0$, on a $c_{0}=1$ et c'est évidemment vrai. Sinon, on écrit (avec la convention $1+p^{0} \mathbb{Z}_{p}=\mathbb{Z}_{p}^{\times}$):

$$
\sum_{\sigma \in 1+p^{l-1} \mathbb{Z} / p^{1} \mathbb{Z}} \kappa(\tilde{\sigma}) \sigma\left(c_{l}\right)=c_{l-1}+\sum_{\sigma \in 1+p^{l-1} \mathbb{Z} / p^{1} \mathbb{Z}}(\kappa(\tilde{\sigma})-1) \sigma\left(c_{l}\right) .
$$

Comme

$$
\sum_{\sigma \in 1+p^{l-1} \mathbb{Z} / p^{1} \mathbb{Z}}(\kappa(\tilde{\sigma})-1) \sigma\left(c_{l}\right) \in A^{00} \mathfrak{F}_{n, r, I}(\operatorname{Spf} A)
$$

d'après le lemme 5.2. On conclût en appliquant l'hypothèse de récurrence à la formule :

$$
\sum_{\sigma \in\left(\mathbb{Z} / p^{1} \mathbb{Z}\right)^{\times}} \kappa(\tilde{\sigma}) \sigma\left(c_{l}\right)=\sum_{\tau \in\left(\mathbb{Z} / p^{l-1} \mathbb{Z}\right)^{\times}} \kappa(\tilde{\tau}) \tau\left(c_{l-1}+\sum_{\sigma \in 1+p^{l-1} \mathbb{Z} / p^{1} \mathbb{Z}}(\kappa(\tilde{\sigma})-1) \sigma\left(c_{l}\right)\right) .
$$

Par conséquent, $e_{c_{n}}(s)$ appartient bien à $\mathfrak{w}_{n, r, I}(\operatorname{Spf} A)$ et c'est de plus un généra-

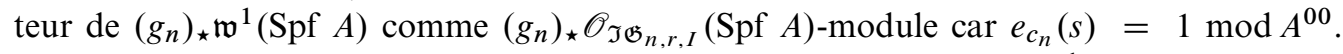
Soit $t \in \mathfrak{w}_{n, r, I}(\operatorname{Spf} A)$. On peut le voir comme un élément de $\left(g_{n}\right)_{\star} \mathfrak{w}_{n, r, I}^{1}(\operatorname{Spf} A)$, et on a $t=\lambda e_{c_{n}}(s)$ avec $\lambda \in\left(g_{n}\right)_{\star} \mathscr{O}_{\mathfrak{I} \mathfrak{G}_{n, r}, I}(\operatorname{Spf} A)$. Mais $\lambda$ est invariant sous $\left(\mathbb{Z} / p^{\mathrm{n}} \mathbb{Z}\right)^{\times}$, donc $\lambda \in A$. Ceci démontre que $\mathfrak{w}_{n, r, I}(\operatorname{Spf} A)$ est libre, engendré par $e_{c_{n}}(s)$.

\subsection{Fonctorialité}

Dans ce numéro, nous montrons que le faisceau est fonctoriel par rapport à l'intervalle $I$, aux entiers $n, r$, et au morphisme de Frobenius.

5.4.1. Fonctorialité en l'intervalle $I$. - Soit $J \subset I$ un sous-intervalle. On a un morphisme naturel $i_{J, I}: \mathfrak{W}_{J}^{0} \rightarrow \mathfrak{W}_{I}^{0}$ et on dispose d'un diagramme commutatif :

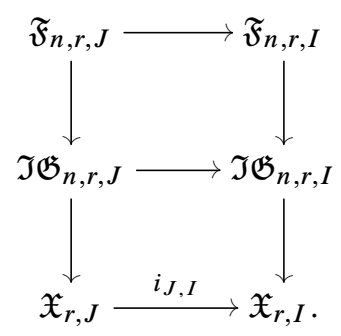


Le morphisme $\mathfrak{F}_{n, r, J} \rightarrow \mathfrak{F}_{n, r, I}$ est équivariant sous l'action du groupe $\mathbb{Z}_{p}^{\times}\left(1+p^{\mathrm{n}} \mathrm{Hdg}^{-\frac{p^{n}}{p-1}} \mathbb{G}_{a}\right)$. Supposons que $n \geq k^{\prime}+2$ (resp. $n \geq k^{\prime}+4$ si $p=2$ ).

Le diagramme ci-dessus induit un morphisme canonique :

$$
i_{J, I}^{\star} \mathfrak{w}_{n, r, I} \rightarrow \mathfrak{w}_{n, r, J}
$$

Proposition 5.1. - Supposons $r \geq 1$ (resp. $r \geq 2$ si $p=2$ ). Le morphisme canonique précédent est un isomorphisme.

Démonstration. - Tensorisons le morphisme $i_{J, I}^{\star} \mathfrak{w}_{n, r, I} \rightarrow \mathfrak{w}_{n, r, J} \operatorname{par}\left(i_{J, I}^{\star} \mathfrak{w}_{n, r, I}\right)^{-1}$. On obtient un morphisme $\mathscr{O}_{\mathfrak{X}_{r, J}} \rightarrow \mathfrak{w}_{n, r, J} \otimes\left(i_{J, I}^{\star} \mathfrak{w}_{n, r, I}\right)^{-1}$, où $\mathfrak{w}_{n, r, I} \otimes\left(i_{J, I}^{\star} \mathfrak{w}_{n, r, J}\right)^{-1}$ est un sous-faisceau du faisceau des fonctions invariantes sous $\mathbb{Z}_{p}^{\times}\left(1+p^{\mathrm{n}} \mathrm{Hdg}^{-\frac{p^{n}}{p-1}} \mathbb{G}_{a}\right)$ sur $\mathfrak{F}_{n, r, J}$, qui vaut $\mathscr{O}_{\mathfrak{X}_{r, J}}$. Le composé

$$
\mathscr{O}_{\mathfrak{X}_{r, J}} \rightarrow \mathfrak{w}_{n, r, J} \otimes\left(i_{J, I}^{\star} \mathfrak{w}_{n, r, I}\right)^{-1} \rightarrow \mathscr{O}_{\mathfrak{X}_{r, J}}
$$

est l'isomorphisme canonique, et donc $i_{J, I}^{\star} \mathfrak{w}_{n, r, I} \rightarrow \mathfrak{w}_{n, r, J}$ est un isomorphisme.

5.4.2. Fonctorialité en $n$. - Supposons $r+k \geq n^{\prime} \geq n$. On a un diagramme commutatif

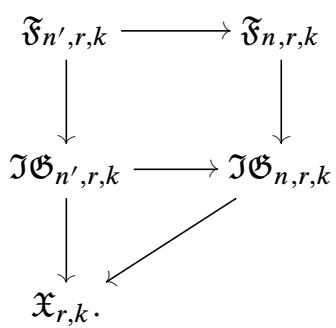

Le groupe $\mathbb{Z}_{p}^{\times}\left(1+p^{\mathrm{n}} \operatorname{Hdg}^{-\frac{p^{n}}{p-1}} \mathbb{G}_{a}\right)$ agit sur $\mathfrak{F}_{n, r, I}$ et $\mathbb{Z}_{p}^{\times}\left(1+p^{n^{\prime}} \operatorname{Hdg}^{-\frac{p^{n^{\prime}}}{p-1}} \mathbb{G}_{a}\right)$ agit sur $\mathfrak{F}_{n^{\prime}, r, I}$. Le morphisme $\mathfrak{F}_{n^{\prime}, r, I} \rightarrow \mathfrak{F}_{n, r, I}$ est équivariant sous le morphisme évident $\mathbb{Z}_{p}^{\times}\left(1+p^{n^{\prime}} \operatorname{Hdg}^{-\frac{p^{n^{\prime}}}{p-1}} \mathbb{G}_{a}\right) \rightarrow \mathbb{Z}_{p}^{\times}\left(1+p^{\mathrm{n}} \operatorname{Hdg}^{-\frac{p^{\mathrm{n}}}{p-1}} \mathbb{G}_{a}\right)$.

Supposons $n \geq k^{\prime}+2$ et $r \geq 1$ (resp. $n \geq k^{\prime}+4$ et $r \geq 2$ si $p=2$ ). Le diagramme ci-dessus induit un morphisme canonique :

$$
\mathfrak{w}_{n, r, I} \rightarrow \mathfrak{w}_{n^{\prime}, r, I}
$$

Proposition 5.2. - Le morphisme canonique précédent est un isomorphisme.

Démonstration. - Tensorisons le morphisme $\mathfrak{w}_{n, r, I} \rightarrow \mathfrak{w}_{n^{\prime}, r, I}$ par $\mathfrak{w}_{n, r, I}^{-1}$. On obtient un morphisme $\mathscr{O}_{\mathfrak{X}_{r, I}} \rightarrow \mathfrak{w}_{n^{\prime}, r, I} \otimes \mathfrak{w}_{n, r, I}^{-1}$, où $\mathfrak{w}_{n^{\prime}, r, I} \otimes \mathfrak{w}_{n, r, I}^{-1}$ est un sous-faisceau du faisceau des fonctions invariantes sous $\mathbb{Z}_{p}^{\times}\left(1+p^{n^{\prime}} \mathrm{Hdg}^{-\frac{p^{n^{\prime}}}{p-1}} \mathbb{G}_{a}\right)$ sur $\mathfrak{F}_{n^{\prime}, r, I}$, qui vaut $\mathscr{O}_{\mathfrak{X}_{r, I}}$. Le composé

$$
\mathscr{O}_{\mathfrak{X}_{r, I}} \rightarrow \mathfrak{w}_{n^{\prime}, r, I} \otimes \mathfrak{w}_{n, r, I}^{-1} \rightarrow \mathscr{O}_{\mathfrak{X}_{r, I}}
$$

est l'isomorphisme canonique, et donc $\mathfrak{w}_{n, r, I} \rightarrow \mathfrak{w}_{n^{\prime}, r, I}$ est un isomorphisme.

On écrit donc dorénavant $\mathfrak{w}_{n, r, I}=\mathfrak{w}_{r, I}$. 
5.4.3. Fonctorialité à la restriction. - Soit $r^{\prime} \geq r$. On a un diagramme commutatif :

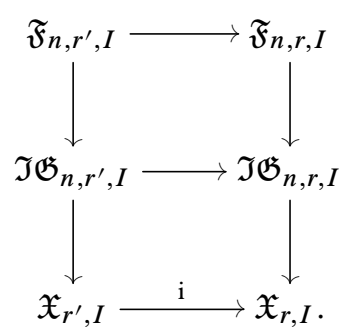

Le morphisme $\mathfrak{F}_{n, r^{\prime}, I} \rightarrow \mathfrak{F}_{n, r, I}$ est équivariant sous l'action du groupe $\mathbb{Z}_{p}^{\times}\left(1+p^{\mathrm{n}} \operatorname{Hdg}^{-\frac{p^{\mathrm{n}}}{p-1}} \mathbb{G}_{a}\right)$. Supposons que $r^{\prime} \geq r \geq 1$ et $n \geq k^{\prime}+2$ (resp. $r^{\prime} \geq r \geq 2$ et $n \geq k^{\prime}+4$ si $p=2$ ). On dispose donc d'un morphisme $i^{\star} \mathfrak{w}_{r, I} \rightarrow \mathfrak{w}_{r^{\prime}, I}$.

Proposition 5.3. - Le morphisme $i^{\star} \mathfrak{w}_{r, I} \rightarrow \mathfrak{w}_{r^{\prime}, I}$ est un isomorphisme.

Démonstration. - C'est analogue à la démonstration de la proposition 5.2.

Dorénavant, on écrit donc $\mathfrak{w}_{r, I}=\mathfrak{w}_{I}$.

5.4.4. Fonctorialité pour le morphisme de Frobenius. - L'isogénie canonique $F: E \rightarrow$ $E / H_{1}:=E^{\prime}$ induit un morphisme $\phi: \mathfrak{X}_{r, I} \rightarrow \mathfrak{X}_{r-1, I}$. Cette application se relève en une application $\phi: \mathfrak{I G}_{n, r, I} \rightarrow \mathfrak{I G}_{n, r-1, I}$ si $n \leq k+r-1$ (voir la proposition 3.6). On a donc une application $\mathfrak{I G}_{n+1, r, I} \rightarrow \mathfrak{I G}_{n, r, I}$ obtenue en composant la projection naturelle $\mathfrak{I G}_{n+1, r, I}$ et le morphisme $F$. Indiquons une description directe du morphisme précédent.

Le morphisme $F$ induit une surjection $F: H_{n+1}(E) \rightarrow H_{\mathrm{n}}\left(E^{\prime}\right)$, et donc une injection $F^{D}: H_{\mathrm{n}}^{D}\left(E^{\prime}\right) \rightarrow H_{n+1}^{D}(E)$ des duaux de cartier. Le morphisme $\mathfrak{I G}_{n+1, r, I} \rightarrow \mathfrak{I G}_{n, r-1, I}$ associe à un morphisme $\psi: \mathbb{Z} / p^{n+1} \mathbb{Z} \rightarrow H_{n+1}^{D}(E)$ le morphisme $\psi^{\prime}: \mathbb{Z} / p^{n} \mathbb{Z} \rightarrow H_{n}^{D}\left(E^{\prime}\right)$ qui fait commuter le diagramme :

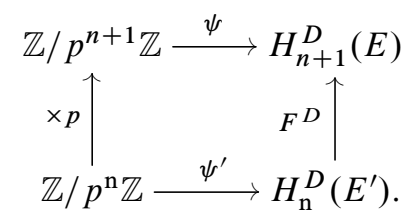

On a alors d'un diagramme commutatif :

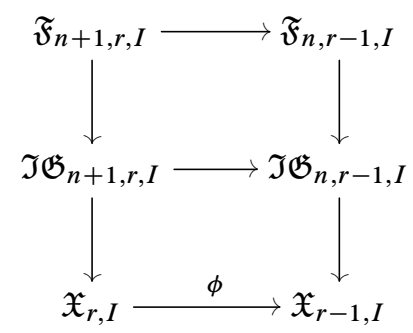


où le morphisme $\mathfrak{F}_{n+1, r, I} \rightarrow \mathfrak{F}_{n, r-1, I}$ est obtenu à l'aide du diagramme commutatif :

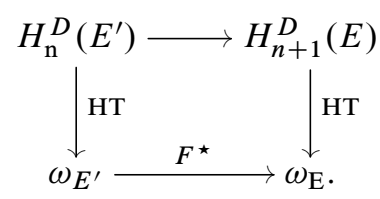

Pour toute $\mathbb{Z}_{p}$-algèbre plate $R$, on associe à $\omega \in \mathfrak{F}_{n+1, r, I}(R)$, la forme différentielle $p \omega$, qui est vue dans $\omega_{E^{\prime}}$ et qui appartient bien à $\mathfrak{F}_{n, r-1, k}(R)$.

Le morphisme $\mathfrak{F}_{n+1, r, k} \rightarrow \mathfrak{F}_{n, r-1, k}$ est équivariant sous le morphisme de groupes $\mathbb{Z}_{p}^{\times}\left(1+p^{n+1} \operatorname{Hdg}(E)^{-\frac{p^{n+1}}{p-1}} \mathbb{G}_{a}\right) \rightarrow \mathbb{Z}_{p}^{\times}\left(1+p^{\mathrm{n}} \operatorname{Hdg}\left(E^{\prime}\right)^{-\frac{p^{n}}{p-1}} \mathbb{G}_{a}\right)$. Supposons $r \geq 2$ et $n \geq k^{\prime}+2$ (resp. $r \geq 3$ et $n \geq k^{\prime}+4$ si $p=2$ ). On dispose d'un morphisme $\phi^{\star} \mathfrak{w}_{I} \rightarrow \mathfrak{w}_{I}$.

Proposition 5.4. - Le morphisme $\phi^{\star} \mathfrak{w}_{I} \rightarrow \mathfrak{w}_{I}$ est un isomorphisme.

Démonstration. - C'est analogue à la démonstration de la proposition 5.2.

\section{Perfectisation}

Dans la partie précédente, nous avons expliqué la construction de faisceaux inversibles $\omega_{[0, \infty[}^{\kappa}$. Notre but est désormais de prolonger ces faisceaux à l'infini. La difficulté est la suivante : sur $[0, \infty[$ on a utilisé l'analyticité locale du caractère universel. A l'infini, le caractère cesse d'être localement analytique. Ceci est cependant compensé par le fait qu'un sous-groupe canonique d'ordre infini existe. Nous allons considérer un pro-revêtement d'un voisinage strict de la courbe modulaire sur lequel la tour d'Igusa d'ordre infini existe. Ceci nous permettra de définir le faisceau des formes surconvergentes perfectisé de façon uniforme sur ce pro-revêtement. Ensuite nous le descendrons au voisinage strict de la courbe modulaire en utilisant des traces de Tate.

\subsection{Résultats principaux}

Le théorème qui suit est le résultat principal de cette partie. Il rassemble le théorème 6.4 et la proposition 6.8 .

ThÉORÈme 6.1. - Supposons $r \geq 3$ si $p \neq 2$ et $r \geq 5$ si $p=2$, alors on possède un faisceau inversible $\mathfrak{w}_{[p, \infty]}$ sur $\mathfrak{X}_{r,[p, \infty]}$. Ce faisceau jouit des propriétés suivantes :

1. Pour tout intervalle $J=\left[p^{k}, p^{k^{\prime}}\right] \subset\left[p, \infty\left[\right.\right.$ et $r+k \geq k^{\prime}+2$ (resp. $r+k \geq k^{\prime}+4$ si $p=2$ ), l'image inverse de $\mathfrak{w}_{[p, \infty]}$ dans $\mathfrak{X}_{r, J}$ est canoniquement isomorphe au faisceau $\mathfrak{w}_{J}$ du théorème 5.1 .

2. La restriction de $\mathfrak{w}_{[p, \infty]}$ à $\mathfrak{X}_{r,\{\infty\}}$ vaut le faisceau $\mathfrak{w}_{\{\infty\}}$ du théorème 4.1.

3. On a un opérateur de Frobenius :

$$
i^{\star} \mathfrak{w}_{[p, \infty]} \simeq \phi^{\star} \mathfrak{w}_{[p, \infty]}
$$

où $i: \mathfrak{X}_{r+1,[p, \infty]} \rightarrow \mathfrak{X}_{r,[p, \infty]}$ est le morphisme d'inclusion et $\phi: \mathfrak{X}_{r+1,[p, \infty]} \rightarrow \mathfrak{X}_{r,[p, \infty]}$ est le Frobenius.

Il entraîne immédiatement le résultat suivant qui est le résultat principal de ce travail : 
ThÉORÈme 6.2. - Pour tout $r \geq 3$ (resp. $r \geq 5$ si $p=2$ ), on possède un faisceau inversible $\omega_{[0, \infty]}^{\kappa}$ sur l'espace $\mathcal{M}_{r,[0, \infty]}$, ainsi qu'un sous-faisceau $\omega_{[0, \infty]}^{\kappa,+}$ de $\mathscr{O}_{\mathcal{M}_{r,[0, \infty]}^{+}}$-modules. Les propriétés suivantes sont vérifiées :

1. La restriction du sous-faisceau $\omega_{[0, \infty]}^{\kappa,+}$ à la composante libre $\mathscr{W}_{[0, \infty]}^{0}$ est un faisceau inversible de $\mathscr{O}_{\mathscr{C}_{r,[0, \infty]}^{+}}^{+}$-module.

2. Pour tout caractère localement algébrique $\chi . k: \mathbb{Z}_{p}^{\times} \rightarrow \mathbb{C}_{p}^{\times}$avec $\chi$ un caractère fini et $k \in \mathbb{Z}$ identifié à un point de $\left.\mathscr{W}_{,} \omega_{[0, \infty]}^{\kappa}\right|_{\{\chi . k\}}=\omega^{\mathrm{k}}(\chi)$ est le faisceau usuel des formes modulaires de poids $k$ et nebentypus $\chi$.

3. Pour tout caractère $\bar{\kappa}_{\chi}: \mathbb{Z}_{p}^{\times} \rightarrow \mathbb{F}_{p}[[T]]^{\times}$identifié à un point du bord de W, $\left.\omega_{[0, \infty]}^{\kappa}\right|_{\left\{\bar{\kappa}_{\chi}\right\}}=\omega^{\bar{\kappa}_{\chi}}$ où $\omega^{\bar{\kappa}_{\chi}}$ est le faisceau des formes surconvergentes en caractéristique $p$, de poids $\bar{\kappa}_{\chi}$ (voir le numéro 4.4.5).

4. Lemorphisme de Frobenius $\phi: M_{r+1,[0, \infty]} \rightarrow M_{r,[0, \infty]}$ et l'inclusioni $: M_{r+1,[0, \infty]} \rightarrow$ $\mathcal{M}_{r,[0, \infty]}$ induisent des isomorphismes $\phi^{\star} \omega_{[0, \infty]}^{\kappa} \simeq i^{\star} \omega_{[0, \infty]}^{\kappa}$. Si on se restreint à $\omega_{[0, \infty]}^{0}$, on a même des isomorphismes au niveau entier $\phi^{\star} \omega_{[0, \infty]}^{\kappa,+} \simeq i^{\star} \omega_{[0, \infty]}^{\kappa,+}$.

Démonstration. - Sur $\mathfrak{M}_{r,[p, \infty]}$ on possède le faisceau $\mathfrak{w}_{I} \otimes \mathfrak{w}^{f}$ où $\mathfrak{w}_{I}$ est donné par le théorème 6.1 et $\mathfrak{w}^{f}$ est construit au numéro 6.8. Sa fibre générique est par définition $\left(\omega_{[p, \infty]}^{\kappa}, \omega_{[p, \infty]}^{\kappa,+}\right)$. Ce faisceau se recolle avec le faisceau $\left(\omega_{[0, \infty[}^{\kappa}, \omega_{[0, \infty[}^{\kappa,+}\right)$ du théorème 5.2.

Ce théorème permet de construire la courbe de Hecke adique. On renvoie à l'appendice $B$ pour les définitions précises de variété spectrale et variété de Hecke. Soit $f_{r}: \mathcal{M}_{r,[0, \infty]} \rightarrow W_{W}$ le morphisme structural et $\Omega_{r}=\left(f_{r}\right)_{\star} \omega_{[0, \infty]}^{\kappa}$ le faisceau des familles de formes $r$-surconvergentes sur $\mathscr{W}$. Le théorème qui suit est démontré au numéro 6.9.

THÉORÈme 6.3. - On possède sur $\Omega_{r}$ une action compacte de l'opérateur $U_{p}$ et une action de l'algèbre de Hecke commutative $H$ engendrée par les opérateurs de Hecke $T_{\ell}$ pour $\ell$ un nombre premier ne divisant pas $N$. En conséquence,

1. L'opérateur $U_{p}$ possède une série caractéristique $\mathscr{P}(X)$ à coefficients dans $\Lambda$, qui coüncide avec la série caractéristique de Coleman.

2. La spécialisation en un point $\left\{\bar{\kappa}_{\chi}\right\} \hookrightarrow \mathscr{W}$ de $\mathscr{P}(X)$ est la série caractéristique de $U_{p}$ agissant sur $M_{\bar{\kappa}_{\chi}}^{\dagger}$.

3. On peut définir la variété spectrale $w: Z \rightarrow$ W. C'est le lieu des zéros de $\mathscr{P}(X)$ dans $\omega \times \mathbb{A}^{1}$. Le morphisme $w$ est localement quasi-fini, plat et partiellement propre sur $\mathscr{W}$. En particulier, localement sur $\mathscr{Z}$ et $\mathscr{W}$, le morphisme $w$ est fini et plat.

4. On possède une variété de Hecke $\mathcal{E} \rightarrow \mathscr{Z} \rightarrow \mathcal{W}$ qui est localement quasi-finie et sans torsion sur $\mathscr{W}$. Les points de $\mathcal{E}$ sont des triplets $\left(\kappa, \alpha^{-1}, \lambda\right)$ où $\kappa \in \mathcal{W}$ est un poids, $\alpha$ est une valeur propre (non nulle) de $U_{p}$ agissant sur $\left.\Omega_{r}\right|_{\kappa}$ et $\lambda$ est un système de valeurs propres de $H$ agissant sur $\left(\left.\Omega_{r}\right|_{\kappa}\right)^{U_{p}=\alpha}$.

\subsection{La tour anticanonique}

Soit $I=\left[p^{k}, p^{k^{\prime}}\right]$ un sous-intervalle de $[1, \infty]$ avec $k, k^{\prime} \in \mathbb{Z}_{\geq 0} \cup\{\infty\}$. Rappelons qu'on a des morphismes finis et plats de Frobenius (voir la proposition 3.3) :

$$
\phi: \mathfrak{X}_{r+1, I} \rightarrow \mathfrak{X}_{r, I} .
$$


Soit $\mathfrak{X}_{\infty, I}=\lim _{r} \mathfrak{X}_{r, I}$ le schéma formel $T$-adique obtenu par limite inverse. L'existence de ce schéma formel est assuré car les morphismes de transition sont affines. Si Spf $R_{r}$ est un ouvert affine de $\mathfrak{X}_{r, I}$ et si Spf $R_{r^{\prime}}$ désigne son image inverse par le morphisme $\phi^{r^{\prime}-r}$ pour tout $r^{\prime} \geq r$, alors l'image inverse de $\operatorname{Spf} R_{r}$ dans $\mathfrak{X}_{\infty, I}$ est $\operatorname{Spf} R_{\infty}$ où $R_{\infty}$ est la complétion $T$-adique de la limite inductive colim $r_{r^{\prime} \geq r} R_{r^{\prime}}$. C'est la tour anticanonique (voir [26], sect. 3). Pour tout $n \leq r+k$, on possède aussi une tour d'Igusa partielle $\mathfrak{I G}_{n, r, I}$ et on a un morphisme de Frobenius compatible avec le morphisme au-dessus (voir la proposition 3.6) :

$$
\phi: \mathfrak{I G}_{n, r+1, I} \rightarrow \mathfrak{I G}_{n, r, I}
$$

En passant à la limite projective on obtient le schéma formel $T$-adique $\mathfrak{I G}_{n, \infty, I} \rightarrow \mathfrak{X}_{\infty, I}$. Le morphisme précédent est affine et entier. En faisant varier $n$, on obtient finalement une tour de schémas formels $\mathfrak{I G}_{n+1, \infty, I} \rightarrow \mathfrak{I G}_{n, \infty, I} \rightarrow \cdots \rightarrow \mathfrak{X}_{\infty, I}$. On note alors $\mathfrak{I G} \mathfrak{G}_{\infty, \infty, I}$ la limite projective, dans la catégorie des schémas formels $T$-adiques de cette tour. Le groupe $\mathbb{Z}_{p}^{\times}$ agit sur $\mathfrak{I G}_{\infty, \infty, I}$.

Au-dessus de $\mathfrak{X}_{\infty, I}$, on a une chaîne d'isogénies entre schémas semi-abéliens :

$$
\cdots E_{r} \stackrel{F_{r}}{\rightarrow} E_{r-1} \cdots
$$

où $E_{r}$ provient de $\mathfrak{X}_{r, I}$. Soit $\operatorname{Hdg}_{r}$ l'idéal de Hodge associé à $E_{r}$. On a $\operatorname{Hdg}_{r+1}^{p}=\operatorname{Hdg}_{r}$ d'après le corollaire A.2.

Pour tout $n \leq r+k$, on dispose (voir le numéro 3.2.2) d'un morphisme de trace $\operatorname{Tr}_{\mathfrak{I G}}: \mathscr{O}_{\mathfrak{I} \mathfrak{G}_{n, r, I}} \rightarrow \mathscr{O}_{\mathfrak{I G}_{n-1, r, I}}$, fonctoriel pour les applications de Frobenius. Par passage à la limite inductive et complétion, on obtient un morphisme $\operatorname{Tr}_{\mathfrak{I G}}: \mathscr{O}_{\mathfrak{I} \mathfrak{G}_{n, \infty, I}} \rightarrow \mathscr{O}_{\mathfrak{I} \mathfrak{G}_{n-1, \infty, I}}$. Nous utilisons pour simplifier la notation $\operatorname{Tr}_{\mathfrak{I} \mathfrak{G}}$ pour tous les morphismes de trace dans la tour d'Igusa partielle. Cette notation omet la référence à la source et au but de ces morphismes.

Proposition 6.1. - On a $\operatorname{Hdg}_{r} \mathscr{O}_{\mathfrak{I} \mathfrak{G}_{n-1, \infty, I}} \subset \operatorname{Tr}_{\mathfrak{I G}}\left(\mathscr{O}_{\mathfrak{I G} \mathfrak{G}_{n, \infty}, I}\right)$ pour tout $r \in \mathbb{Z}_{\geq 1}$.

Démonstration. - D'après la proposition 3.5, on a $\operatorname{Hdg}_{\mathrm{r}} p^{n-1} \subset \operatorname{Tr}_{\mathfrak{I} \mathfrak{G}}\left(\mathscr{O}_{\mathfrak{I} \mathfrak{G}_{n, r, I}}\right)$. Il en résulte que pour tout $r \geq n, \operatorname{Hdg}_{r-n+1}=\operatorname{Hdg}_{\mathrm{r}} p^{n-1} \subset \operatorname{Tr}_{\mathfrak{I G}}\left(\mathscr{O}_{\mathfrak{I G}}{ }_{n, \infty, I}\right)$.

REMARQUe 6.1. - Le fait de passer à la tour anticanonique a donc tué la ramification de la tour d'Igusa. C'est naturel car la tour anti-canonique $\mathfrak{X}_{\infty, I}$ est « relativement perfectoïde» au-dessus de l'espace des poids.

\subsection{Traces de Tate}

L'objectif de cette section est de construire des traces de Tate qui permettent de redescendre le long de la tour anti-canonique. Notons $h_{r}: \mathfrak{X}_{\infty, I} \rightarrow \mathfrak{X}_{r, I}$ la projection évidente. Le résultat principal de cette section est le suivant.

Proposition 6.2. - Pour tout $r \geq 1$, on a des traces de Tate:

$$
\operatorname{Tr}_{\mathrm{r}}:\left(h_{r}\right)_{\star} \mathscr{O}_{\mathfrak{X}_{\infty, I}}[1 / T] \rightarrow \mathscr{O}_{\mathfrak{X}_{r, I}}[1 / T]
$$

telles que pour tout $f \in \mathscr{O}_{\mathfrak{X}_{\infty, I}}[1 / T], f=\lim _{r \rightarrow \infty} \operatorname{Tr}_{\mathrm{r}} f$. De plus, pour tout $r \geq 1$ (resp. $r \geq 2$ si $p=2$ ),

$$
\operatorname{Tr}_{\mathrm{r}}\left(\left(h_{r}\right)_{\star} \mathscr{O}_{\mathfrak{X}_{\infty, I}}\right) \subset T^{-1} \mathscr{O}_{\mathfrak{X}_{r, I, \infty}}
$$


6.3.1. Un lemme sur la trace. - Le lemme suivant est la généralisation directe du corollaire III.2.21 de [26].

Lemme 6.1. - Soit $n \geq 1$ un entier. Soit $S$ une $\mathbb{Z}_{p}$ algèbre complète, topologiquement de type fini, de dimension $d$, formellement lisse. Soit $B$ une $\mathbb{Z}_{p}[[T]]$-algèbre, sans torsion comme $\mathbb{Z}_{p}[[T]]-$ module, intègre et $T$-adiquement complète. On suppose aussi que B possède un élement $T^{1 / p^{n+1}}$ et que $p \in T B$. Soit $R=S \hat{\otimes}_{\mathbb{Z}_{p}}$. Soit $f \in S$ tel que $\bar{f} \in S / p$ n'est pas diviseur de 0 . Soit $R_{n}=R\left\langle u_{n}\right\rangle /\left(f u_{n}-T^{1 / p^{n}}\right)$. Soit $F: R_{n} \rightarrow R_{n+1}$ une application B-linéaire telle que modulo $p T^{-1 / p^{n}}, F$ est donnée par $F\left(u_{n}\right)=u_{n+1}^{p}$ et par le Frobenius sur $S / p$. Alors $R_{n+1}[1 / T]$ est une algèbre finie et plate au-dessus de $R_{n}[1 / T]$ et $\operatorname{Tr}_{\mathrm{F}}\left(R_{n+1}\right) \subset p^{\mathrm{d}} T^{-\frac{2 d+1}{p^{n}}} R_{n}$.

Démonstration. - Commençons par remarquer que $R_{n}$ et $R_{n+1}$ sont des $\mathbb{Z}_{p}$-algèbres plates. En effet, $S \hat{\otimes}_{\mathbb{Z}_{p}} B=R$ est plate sur $\mathbb{Z}_{p}$ et donc $R\left\langle u_{n}\right\rangle$ aussi. Soit $x, y \in R\left\langle u_{n}\right\rangle$ tels que $p x=y\left(f u_{n}-T^{1 / p^{n}}\right)$. Posons $x=\sum_{k} a_{k} u_{n}^{k}$ et $y=\sum_{k} b_{k} u_{n}^{k}$. On obtient alors $b_{k} f=T^{1 / p^{n}} b_{k+1}+p a_{k+1}$. Si on réduit modulo $T^{1 / p^{n}}$ la relation $b_{k} f=T^{1 / p^{n}} b_{k+1}+p a_{k+1}$, on obtient $b_{k} \in\left(p, T^{1 / p^{n}}\right)$ car $f \otimes 1$ n'est pas diviseur de 0 dans $S / p \otimes_{\mathbb{F}_{p}} B / T^{1 / p^{n}}$. Montrons par récurrence sur $l$ que $b_{k} \in\left(p, T^{l / p^{n}}\right) R$ pour tout $l \geq 1$. Cela montrera que $b_{k} \in p R$, puis que $x \in\left(f u_{n}-T^{1 / p^{n}}\right)$. Supposons donc l'hypothèse vérifiée au cran $l-1$. Réduisant modulo $\left(p, T^{l / p^{n}}\right)$ la relation $b_{k} f=T^{1 / p^{n}} b_{k+1}+p a_{k+1}$ on obtient $b_{k} f=0$ dans $S / p \otimes_{\mathbb{F}_{p}} B /\left(p, T^{l / p^{n}}\right)$ et, comme $f$ n'est pas diviseur de 0 dans $S / p \otimes_{\mathbb{F}_{p}} B /\left(p, T^{l / p^{n}}\right)$ on obtient $b_{k} \in\left(p, T^{l / p^{n}}\right) R$.

Comme le lemme est local sur $\operatorname{Spf} S$, on peut supposer qu'il y a une application Spf $S \rightarrow \operatorname{Spf} \mathbb{Z}_{p}\left\langle Y_{1}, \ldots, Y_{d}\right\rangle$ étale. On peut donc supposer que $S / p$ est, vue comme $S / p$-module via le Frobenius, un $S / p$-module libre de base $Y_{1}^{i_{1}}, \ldots, Y_{d}^{i_{d}}$ avec $0 \leq i_{k} \leq p-1$. Soit maintenant l'algèbre $R_{n+1}^{\prime}=R\left\langle v_{\mathrm{n}}\right\rangle /\left(f^{p} v_{n}-T^{1 / p^{n}}\right)$. C'est aussi une $\mathbb{Z}_{p}$-algèbre plate. On a une application $\tau: R_{n+1}^{\prime} \rightarrow R_{n+1}$ donnée par $v_{n} \mapsto u_{n+1}^{p}$. Après inversion de $T$, cette application est un isomorphisme, d'inverse donné par $u_{n+1} \mapsto v_{n} f^{p-1} T^{-\frac{p-1}{p^{n+1}}}$. On en déduit que le morphisme $\tau$ est injectif, de conoyau tué par $T^{\frac{p-1}{p^{n+1}}}$. On va maintenant vérifier que le morphisme $F: R_{n} \rightarrow R_{n+1}$ se factorise en $\tau \circ F^{\prime}$ pour un morphisme $F^{\prime}: R_{n} \rightarrow R_{n+1}^{\prime}$. Pour le voir, il suffit de le vérifier modulo $T^{\frac{p^{\mathrm{n}}-1}{p^{\mathrm{n}}}}$ car toutes les algèbres en jeu sont sans $T$-torsion et $\frac{p^{\mathrm{n}}-1}{p^{\mathrm{n}}}>\frac{p-1}{p^{n+1}}$. Comme $T$ divise $p$, on se ramène à le vérifier pour $F \bmod \frac{p}{T^{1 / p^{\mathrm{n}}}}$, et c'est alors évident. On vérifie de même que $F^{\prime} \bmod \frac{p}{T^{2 / p^{\mathrm{n}}}}$ est l'application qui envoie $u_{n}$ sur $v_{n}$ et qui est le Frobenius absolu sur $S \bmod p$. On en déduit que $R_{n+1}^{\prime} \bmod \frac{p}{T^{2 / p^{\mathrm{n}}}}$ est finie et plate comme $R_{n} / \frac{p}{T^{2 / p^{n}}}$-algèbre, engendrée par $Y_{1}^{i_{1}}, \ldots, Y_{d}^{i_{d}}$, $0 \leq i_{k} \leq p-1$. La même chose est vraie pour $R_{n+1}^{\prime}$ vue comme $R_{n}$-algèbre. Il en résulte que $R_{n+1}^{\prime}=R_{n}\left\langle X_{1}, \ldots, X_{d}\right\rangle /\left(X_{1}^{p}-Y_{1}-\frac{p}{T^{2 / p^{\mathrm{n}}}} P_{1}, \ldots, X_{d}^{p}-Y_{d}-\frac{p}{T^{2 / p^{\mathrm{n}}}} P_{d}\right)$ pour des polynômes $P_{i} \in R_{n}\left\langle X_{1}, \ldots, X_{d}\right\rangle$. On peut alors appliquer le lemme III. 2.20 de [26] pour en déduire que $\operatorname{Tr}_{F^{\prime}}\left(R_{n+1}^{\prime}\right) \subset \frac{p^{d}}{T^{2 d / p^{\mathrm{n}}}} R_{n}$. Il en résulte alors que $\operatorname{Tr}_{\mathrm{F}}\left(R_{n+1}\right) \subset \frac{p^{d}}{T^{2 d+1 / p^{\mathrm{n}}}} R_{n}$.

6.3.2. Extraction de racines de $T$. - Pour appliquer le lemme précédent, nous avons besoin d'extraire des racines de $T$ dans l'espace des poids. Pour tout $k \geq 0$, on note ${ }_{\omega^{0, p^{-k}}}=\operatorname{Spa}\left(\mathbb{Z}_{p}\left[\left[T^{1 / p^{k}}\right]\right], \mathbb{Z}_{p}\left[\left[T^{1 / p^{k}}\right]\right]\right)^{\text {an }}$. Si $k=0$ on note indifféremment $\mathscr{Q}^{0, p^{-0}}$ 
ou W. Pour $k^{\prime} \leq k$, le morphisme évident $\mathbb{Z}_{p}\left[\left[T^{1 / p^{k^{\prime}}}\right]\right] \rightarrow \mathbb{Z}_{p}\left[\left[T^{1 / p^{k}}\right]\right]$ fournit des applications $\omega^{0, p^{-k}} \rightarrow \omega^{0, p^{-k^{\prime}}}$. On note $\mathscr{\omega}_{I}^{0, p^{-k}}$ l'image inverse de $\omega_{I}^{0}$ dans $\omega^{0, p^{-k}}$. On pose $B_{I, p^{-k}}=\mathrm{H}^{0}\left(\mathscr{W}_{I}^{0, p^{-k}}, \mathscr{O}_{\mathscr{W}^{0, p^{-k}}}^{+}\right)$et $\mathfrak{W}_{I}^{0, p^{-k}}=\operatorname{Spf} B_{I, p^{-k}}$. On observe que le morphisme qui envoie $T \operatorname{sur} T^{1 / p^{k}}$ induit un isomorphisme $B_{p^{-k} I} \rightarrow B_{I, p^{-k}}$. Soit $k \geq r+1$. On note $\mathscr{X}_{r, I, p^{-k}}$ le produit fibré $\mathscr{E}_{r, I} \times_{\mathscr{W}_{I}^{0}} \mathscr{W}_{I}^{0, p^{-k}}$. On définit alors $\mathfrak{X}_{r, I, p^{-k}}$ comme le normalisé de $\mathfrak{X}_{r, I}$ dans $\mathscr{Q}_{r, I, p^{-k}}$.

6.3.3. Description de $\mathfrak{X}_{r, I, p^{-k}}$. - On a une chaîne de morphismes affines

$$
\mathfrak{X}_{r, I, p^{-k}} \rightarrow \mathfrak{X}_{r, I} \rightarrow \mathfrak{X}_{I} \rightarrow \mathfrak{X} .
$$

Si Spf $A$ est un ouvert affine de $\mathfrak{X}$, si le faisceau $\omega_{E}$ est trivial sur Spf $A$ et si $\tilde{\mathrm{H} a} \in A$ est un relèvement de l'invariant de Hasse dans la trivialisation, la fibre au-dessus de $\operatorname{Spf} A$ de cette chaîne de morphismes vaut (voir la proposition 3.1) :

$$
A \rightarrow A \hat{\otimes}_{\mathbb{Z}_{p}} B_{I} \rightarrow A \hat{\otimes}_{\mathbb{Z}_{p}} B_{I}\left\langle\frac{T}{\tilde{\mathrm{Ha}}^{p^{r+1}}}\right\rangle \rightarrow A \hat{\otimes}_{\mathbb{Z}_{p}} B_{I, p^{-k}}\left\langle\frac{T^{1 / p^{r+1}}}{\tilde{\mathrm{Ha}}}\right\rangle .
$$

6.3.4. Application du lemme. - On a un carré cartésien (la colonne de gauche est le changement de base, de $\omega_{I}^{0}$ à $\omega_{I, p^{-k}}^{0}$ de la colonne de droite ) :

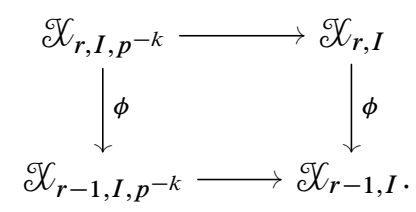

Celui-ci est la fibre analytique d'un diagramme commutatif :

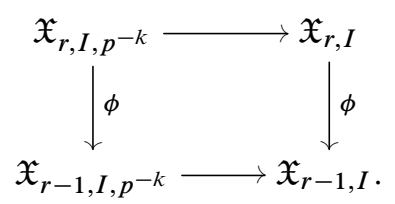

Il en résulte que le morphisme $\phi: \mathfrak{X}_{r, I, p^{-k}} \rightarrow \mathfrak{X}_{r-1, I, p^{-k}}$ est localement donné par un morphisme d'algèbres :

$$
A \hat{\otimes}_{\mathbb{Z}_{p}} B_{I, p^{-k}}\left\langle\frac{T^{1 / p^{\mathrm{r}}}}{\tilde{\mathrm{Ha}}}\right\rangle \rightarrow A \hat{\otimes}_{\mathbb{Z}_{p}} B_{I, p^{-k}}\left\langle\frac{T^{1 / p^{r+1}}}{\tilde{\mathrm{Ha}}}\right\rangle
$$

qui modulo $p T^{-1 / p^{r}}$ provient du morphisme de Frobenius absolu sur $A$.

Corollaire 6.1. - On a

1. pour tout $k \geq r+1, \operatorname{Tr}_{\phi}\left(\mathscr{O}_{\mathfrak{X}_{r, I, p}-k}\right) \subset p T^{-\frac{3}{p^{r}}} \mathscr{O}_{\mathfrak{X}_{r-1, I, p}-k}$,

2. pour tout $k \geq r+1$ et $r^{\prime} \geq r, \operatorname{Tr}_{\phi^{r^{\prime}-r}}\left(\mathscr{O}_{\mathfrak{X}_{r^{\prime}, I, p}-k}\right) \subset p^{r^{\prime}-r} T^{-\frac{3}{p^{\mathrm{r}(p-1)}}} \mathscr{O}_{\mathfrak{X}_{r, I, p}-k}$,

3. pour tout $r^{\prime} \geq r \geq 1$ (resp. $r \geq 2$ si $p=2$ ), $\operatorname{Tr}_{\phi^{r^{\prime}-r}}\left(\mathscr{O}_{\mathfrak{X}_{r^{\prime}, I}}\right) \subset p^{r^{\prime}-r} T^{-1} \mathscr{O}_{\mathfrak{X}_{r, I}}$. 
Démonstration. - Les points 1 et 2 résultent immédiatement du lemme 6.1 appliqué pour $d=1$. Comme le diagramme 6.3.A est cartésien, on déduit de 2 que $\operatorname{Tr}_{\phi^{r^{\prime}-r}}\left(\mathscr{O}_{\mathfrak{x}_{r^{\prime}, I}}\right) \subset$ $p^{r-r^{\prime}} T^{-\frac{3}{p^{\mathrm{r}}(p-1)}} \mathscr{O}_{\mathfrak{X}_{r, I, p}-k} \cap \mathscr{O}_{\mathfrak{X}_{r, I}}[1 / T]$. Pour $r \geq 1$ (resp. $r \geq 2$ ), il suffit de voir que $T^{-\frac{3}{p^{\mathrm{r}}(p-1)}} \mathscr{O}_{\mathfrak{X}_{r, I, p}-k} \cap \mathscr{O}_{\mathfrak{X}_{r, I}}[1 / T] \subset T^{-1} \mathscr{O}_{\mathfrak{X}_{r, I}}$. C'est clair car $\mathfrak{X}_{r, I, p^{-k}}$ est fini sur $\mathfrak{X}_{r, I}$ qui est intégralement clos dans sa fibre analytique et $\frac{3}{p^{\mathrm{r}}(p-1)} \leq 1$.

Corollaire 6.2. - Pour tout $r \geq 1$, on a des traces de Tate :

$$
\operatorname{Tr}_{\mathrm{r}}:\left(h_{r}\right)_{\star} \mathscr{O}_{\mathfrak{X}_{\infty, I}}[1 / T] \rightarrow \mathscr{O}_{\mathfrak{X}_{r, I}}[1 / T]
$$

telles que pour tout $f \in \mathscr{O}_{\mathfrak{X}_{\infty, I}}[1 / T], f=\lim _{r \rightarrow \infty} \operatorname{Tr}_{\mathrm{r}} f$. De plus, pour tout $r \geq 1$ (resp. $r \geq 2$ si $p=2$ ),

$$
\operatorname{Tr}_{\mathrm{r}}\left(\left(h_{r}\right)_{\star} \mathscr{O}_{\mathfrak{X}_{\infty, I}}\right) \subset T^{-1} \mathscr{O}_{\mathfrak{X}_{r, I, \infty}}
$$

Démonstration. - Considérons l'application

$$
\frac{1}{p^{r^{\prime}-r}} \operatorname{Tr}_{\phi^{r-r^{\prime}}}: \mathscr{O}_{\mathfrak{X}_{r^{\prime}, I}}[1 / T] \rightarrow \mathscr{O}_{\mathfrak{X}_{r, I}}[1 / T] .
$$

Cette application envoie $\mathscr{O}_{\mathfrak{X}_{r^{\prime}, I}}$ dans $T^{-1} \mathscr{O}_{\mathfrak{X}_{r, I}}$. On obtient, en passant à la limite inductive, une application $\operatorname{Tr}_{\mathrm{r}}: \operatorname{colim}_{r^{\prime}} \mathscr{O}_{\mathfrak{X}_{r^{\prime}, I}}[1 / T] \rightarrow \mathscr{O}_{\mathfrak{X}_{r, I}, \infty}[1 / T]$. Cette application est uniformément continue. On peut donc la prolonger par continuité en une application $\operatorname{Tr}_{\mathrm{r}}: \mathscr{O}_{\mathfrak{X}_{\infty, I}}[1 / T] \rightarrow \mathscr{O}_{\mathfrak{X}_{r, I}}[1 / T]$.

\subsection{Le faisceau modulaire sur la tour anti-canonique}

Nous allons maintenant pouvoir définir le faisceau des formes surconvergentes sur le perfectisé.

Lemme 6.2. - Le faisceau $\mathscr{O}_{\mathfrak{X}_{\infty, I}}$ est intégralement clos dans $\mathscr{O}_{\mathfrak{X}_{\infty, I}}[1 / T]$.

Démonstration. - Soit $\operatorname{Spf} R$ un ouvert de $\mathfrak{X}_{\infty, I}$. Pour tout $r$ suffisamment grand, Spf $R$ provient d'un ouvert $\operatorname{Spf} R_{r}$ de $\mathfrak{X}_{r, I}$. Soit $f \in R[1 / T]$. On suppose que $f$ vérifie une relation de dépendance intégrale sur $R$. Pour $r$ suffisament grand, $f=f_{r}+h$ avec $f_{r} \in R_{r}[1 / T]$ et $h \in R$. On peut donc supposer que $f \in R_{r}[1 / T]$. Soit $f^{n}+f^{n-1} a_{n-1}+\cdots+a_{0}=0$ une relation de dépendance avec $a_{i} \in R$. En appliquant $\operatorname{Tr}_{r^{\prime}}$ pour $r^{\prime} \geq r$ on obtient $f^{n}+f^{n-1} \operatorname{Tr}_{r^{\prime}}\left(a_{n-1}\right)+\cdots+\operatorname{Tr}_{r^{\prime}}\left(a_{0}\right)=0$ et comme $a_{i} \in R$, pour $r^{\prime}$ suffisamment grand $\operatorname{Tr}_{r^{\prime}}\left(a_{i}\right) \in R \cap T^{-1} R_{r^{\prime}}$. Pour tout $r^{\prime \prime} \geq r^{\prime}$, le morphisme $R_{r^{\prime}} \rightarrow R_{r^{\prime \prime}}$ est fidèlement plat, donc $R_{r^{\prime}} / T \rightarrow R_{r^{\prime \prime}} / T$ est injectif et par conséquent $R_{r^{\prime}} / T \rightarrow R / T$ aussi. Il en résulte que $T \operatorname{Tr}_{r^{\prime}}\left(a_{i}\right) \in T R_{r^{\prime}}$ et donc $\operatorname{Tr}_{r^{\prime}}\left(a_{i}\right) \in R_{r^{\prime}}$. En conclusion, $f_{r}$ vérifie une relation de dépendance sur $R_{r^{\prime}}$, donc $f_{r} \in R_{r^{\prime}}$.

LEMME 6.3. - On a $\left(\mathscr{O}_{\mathfrak{I} \mathfrak{G}_{n, \infty, I}}\right)^{\left(\mathbb{Z} / p^{n} \mathbb{Z}\right)^{\times}}=\mathscr{O}_{\mathfrak{X} \infty, I}$.

Démonstration. - Soit $\operatorname{Spf} R$ un ouvert de $\mathfrak{X}_{\infty, I}$. Soit Spf $R_{n}$ son image inverse dans $\mathfrak{I G}_{n, \infty, I}$. Le morphisme $R[1 / T] \rightarrow R_{n}[1 / T]$ est fini étale, donc $\left(R_{n}\right)^{\left(\mathbb{Z} / p^{n} \mathbb{Z}\right)^{\times}} \subset R[1 / T]$. Comme $R_{n}$ est entier sur $R$, le lemme précédent permet de conclure.

Proposition 6.3. - On $a\left(\mathscr{O}_{\mathfrak{I} G \infty, \infty, I}\right)^{\mathbb{Z}_{p}^{\times}}=\mathscr{O}_{\mathfrak{X}_{\infty, I}}$. 
Démonstration. - Soit Spf $R$ un ouvert affine de $\mathfrak{X}_{\infty, I}$. Soit $x \in\left(\mathscr{O}_{\mathfrak{I} \mathfrak{G}_{\infty, \infty, I}}(\operatorname{Spf} R)\right)^{\mathbb{Z}_{p}^{\times}}$. On a $x=x^{\prime}+T x^{\prime \prime}$ avec $x^{\prime} \in \mathscr{O}_{\mathfrak{I} \mathfrak{G}_{n, \infty}, I}(\operatorname{Spf} A)$ pour $n$ assez grand. Il en résulte que

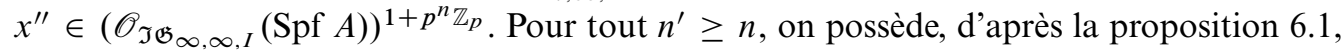
un élément $c_{n^{\prime}} \in \mathscr{O} \mathfrak{I G}_{n^{\prime}, \infty, I}(\operatorname{Spf} A)$ tel que $\operatorname{Tr}_{\mathfrak{I G}_{n^{\prime}, \infty, I} / \mathfrak{I G}_{n, \infty, I}}\left(c_{n^{\prime}}\right)=T$. Par conséquent la cohomologie supérieure de $\left(1+p^{\mathrm{n}} \mathbb{Z} / p^{n^{\prime}} \mathbb{Z}\right)$ agissant sur $\mathscr{O} \mathfrak{I} \mathfrak{G}_{n^{\prime}, \infty, I}(\operatorname{Spf} R)$ est annulée par $T$ (voir [28], sect. 3.2, coro. 1). Pour tout $s$, il existe $n(s) \geq n$ tel que

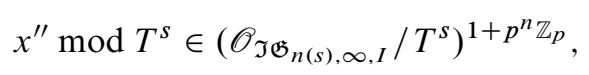

et donc il existe $y_{s} \in \mathscr{O}_{\mathfrak{T} \mathfrak{G}_{n, \infty, I}}(\operatorname{Spf} R)$ tel que $y_{s} \bmod T^{s}=T x^{\prime \prime}$. Il en résulte que $y_{s}$ converge vers un élement $y$ quand $s \rightarrow \infty$ et que $T x^{\prime \prime}=y$. Il en résulte que $x \in \mathscr{O}_{\mathfrak{J G}}{ }_{n, \infty, I}(\operatorname{Spf} A)$ et on conclut par le lemme précédent.

On définit alors le faisceau $\mathfrak{w}_{I}^{\text {perf }}$ comme le sous-faisceau de $\mathscr{O}_{\mathfrak{I} \mathfrak{G}_{\infty, \infty, I}}$ des sections qui se transforment selon le caractère $\kappa^{-1}$ pour l'action du groupe $\mathbb{Z}_{p}^{\times}$.

Proposition 6.4. - Le faisceau $\mathfrak{w}_{I}^{\text {perf }}$ est un faisceau localement libre de rang 1.

Démonstration. - Soit $\operatorname{Spf} A$ un ouvert affine de $\mathfrak{X}_{\infty, I}$. On suppose que $\operatorname{Hdg}_{1}$ est principal sur $\operatorname{Spf} A$, engendré par un élément H̃a 1 . Grâce à la proposition 6.1, on peut trouver une suite d'éléments $c_{n} \in \tilde{\mathrm{Ha}_{1}}{ }_{1}^{-1} \mathscr{O}_{\mathfrak{I G}}{ }_{n, \infty, I}(A)$ tels que $\operatorname{Tr}_{\mathfrak{I} \mathfrak{G}}\left(c_{n}\right)=c_{n-1}, c_{1}=\frac{1}{p-1}, c_{0}=1$.

Posons alors $b_{0}=1$ et pour tout $n \geq 1, b_{n}=\sum_{\sigma \in\left(\mathbb{Z} / p^{n} \mathbb{Z}\right)^{\times}} \kappa(\tilde{\sigma}) \sigma \cdot c_{n}$, où $\tilde{\sigma}$ est un relèvement de $\sigma$ dans $\mathbb{Z}_{p}^{\times}$. D'après le lemme 2.3, on a $\kappa\left(1+p^{\mathrm{n}} \mathbb{Z}_{p}\right) \subset 1+T^{\mathrm{n}} \mathscr{O}_{\mathfrak{W}_{I}}$ (resp. $1+T^{n-1} \mathscr{O}_{\mathfrak{W}_{I}}$ si $p=2$ ) et $\kappa\left(\mathbb{Z} / q \mathbb{Z}^{\times}\right)=1$. Un autre choix de relèvement $\tilde{\sigma}^{\prime}$ donnerait un élément $b_{n}^{\prime}$ et :

$$
\begin{array}{rlrl}
b_{n}^{\prime}-b_{n} & \in \frac{T^{n}}{\tilde{\mathrm{Ha}}{ }_{1}} \mathscr{O}_{\mathfrak{I} \mathfrak{G}_{n, \infty, I}}(A) & \text { si } p \neq 2 \text { et } n \geq 2, \\
b_{n}^{\prime}-b_{n} \in \frac{T^{n-1}}{\tilde{\mathrm{Ha}}{ }_{1}} \mathscr{O}_{\mathfrak{I G} \mathfrak{G}_{n, \infty, I}(A)} & \text { si } p=2 \text { et } n \geq 2, \\
b_{1}-b_{1}^{\prime} \in T A . &
\end{array}
$$

On vérifie comme dans la démonstration du théorème 4.1 que :

$$
\begin{array}{rlrl}
b_{n}-b_{n-1} & \in\left(T^{n-1} \tilde{\mathrm{Ha}}_{1}^{-1}\right) \mathscr{O}_{\mathfrak{I G}}{ }_{n, \infty, I}(A) & \text { si } n \geq 2 \text { et } p \neq 2, \\
b_{n}-b_{n-1} & \in\left(T^{n-2} \tilde{\mathrm{Ha}}_{1}^{-1}\right) \mathscr{O}_{\mathfrak{I G}}{ }_{n, \infty, I}(A) & \text { si } n \geq 3 \text { et } p=2, \\
b_{1}-1 & \in T A, \\
b_{2}-1 & \in T \tilde{\mathrm{Ha}}_{1}^{-1} \mathscr{O}_{\mathfrak{I G}_{2, \infty, I}}(A) . &
\end{array}
$$

La suite $b_{n}$ converge donc vers un élément $b_{\infty} \in \mathscr{O}_{\mathfrak{I G} \infty, \infty, I}(A)$ et $b_{\infty}=1 \bmod \frac{T}{\tilde{\mathrm{Ha}}_{1}}$ est un élément inversible de $\mathscr{O}_{\mathfrak{I} \mathfrak{G}_{\infty, \infty, I}}(A)$. 11 résulte alors de la proposition 6.3 que $\mathfrak{w}_{I}^{\text {perf }}(A)=$ $b_{\infty} \cdot A$.

Proposition 6.5. - Soit $J \subset$ I un sous-intervalle et $i_{J, I}: \mathfrak{X}_{\infty, J} \hookrightarrow \mathfrak{X}_{\infty, I}$ le morphisme naturel. On a la compatibilité au changement de base :

$$
i_{J, I}^{\star} \mathfrak{w}_{I}^{\text {perf }}=\mathfrak{w}_{J}^{\text {perf }} .
$$

Démonstration. - Analogue à la démonstration de la proposition 5.2. 


\subsection{Comparaison avec le faisceau $\mathfrak{w}_{I}$}

Dans ce numéro, $I=\left[p^{k}, p^{k^{\prime}}\right]$ avec $k, k^{\prime} \in \mathbb{Z}_{\geq 0}$. On suppose $k+r \geq k^{\prime}+1$ (resp. $k+r \geq k^{\prime}+3$ si $p=2$ ) et $r \geq 1$ (resp. $r \geq 2$ si $p=2$ ). Sur $\mathfrak{X}_{r, I}$, on possède, d'après le théorème 5.1, un faisceau $\mathfrak{w}_{I}$. Le but de cette section est de démontrer que son image inverse sur la tour anti-canonique est le faisceau $\mathfrak{w}_{I}^{\text {perf }}$.

Au-dessus de $\mathfrak{X}_{\infty, I}$, on a une chaine d'isogénies

$$
\cdots E_{r} \stackrel{F_{r}}{\rightarrow} E_{r-1} \rightarrow \cdots
$$

où $E_{r}$ est le schéma semi-abélien qui provient de $\mathfrak{X}_{r, I}$. On note $C_{n, r} \hookrightarrow E_{r}\left[p^{n}\right]$ le schéma en groupes $\operatorname{Ker}\left(F_{r+n}^{D}: E_{r} \rightarrow E_{r+n}\right)$. Clairement, $C_{n, r}=H_{\mathrm{n}}\left(E_{r+n}\right)^{D}$. L'isogénie $F_{r}: E_{\mathrm{r}} \rightarrow E_{r-1}$ induit un morphisme $C_{n, r} \rightarrow C_{n, r-1}$ qui est un isomorphisme génériquement. Au-dessus de $\mathfrak{I G}_{n, \infty, I}$, on possède un morphisme universel $\mathbb{Z} / p^{n} \mathbb{Z} \rightarrow H_{n}\left(E_{r}\right)^{D}$ pour tout $r \geq n-k$. De plus, l'application $C_{n, r} \rightarrow E_{r}\left[p^{n}\right] / H_{n}\left(E_{r}\right) \simeq H_{n}\left(E_{r}\right)^{D}$ est clairement un isomorphisme générique. En composant, on possède donc une application $\mathbb{Z} / p^{n} \mathbb{Z} \rightarrow C_{n, r}$ pour tout $r \geq n-k$. En utilisant les morphismes $C_{n, r} \rightarrow C_{n, r-1}$ on en déduit l'existence de morphismes $\mathbb{Z} / p^{n} \mathbb{Z} \rightarrow C_{n, r}$ pour tout $n$ et $r$ qui sont des isomorphismes génériques. En passant à la limite projective, on obtient un morphisme $\mathbb{Z}_{p} \rightarrow \lim _{n} C_{n, r}$. Soit HT ${ }^{\text {un }}$ l'image de $1 \in \mathbb{Z}_{p} \simeq \lim _{n} C_{n, r}$ par l'application de Hodge-Tate $\lim _{n} E_{r}\left[p^{n}\right] \rightarrow \omega_{E_{r}}$. Par définition, $\mathrm{HT}^{\mathrm{un}}$ fournit une application $\mathbb{Z}_{p}^{\times}$-équivariante :

$$
\mathfrak{I G}_{\infty, \infty, I} \rightarrow \mathfrak{F}_{n, r, I}
$$

qui s'insère dans le diagramme commutatif :

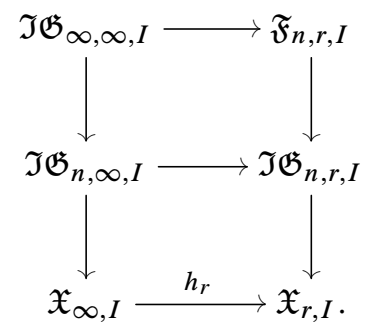

Proposition 6.6. - On dispose d'un isomorphisme canonique $\mathfrak{w}_{I}^{\text {perf }} \simeq\left(h_{r}\right)^{\star} \mathfrak{w}_{I}$.

Démonstration. - On possède clairement une application injective $:\left(h_{r}\right)^{\star} \mathfrak{w}_{I} \rightarrow \mathfrak{w}_{I}^{\text {perf }}$. De plus, $\mathfrak{w}_{I}^{\text {perf }} \otimes\left(h_{r}\right)^{\star} \mathfrak{w}_{I}^{-1} \subset\left(\mathscr{O}_{\mathfrak{I} \mathfrak{G}_{\infty, \infty, I}}\right)^{\mathbb{Z}_{p}^{\times}}=\mathscr{O}_{\mathfrak{X}_{\infty, I}}$ d'après la proposition 6.3.

Corollaire 6.3. - On dispose d'une trace de Tate $\operatorname{Tr}_{r}:\left(h_{r}\right)_{\star} \mathfrak{w}_{I}^{\text {perf }} \rightarrow \mathfrak{w}_{I}\left[T^{-1}\right]$. Cette trace est fonctorielle en l'intervalle I.

\subsection{Descente}

Dans cette section, fixons $I=[p, \infty]$. Nous allons montrer que le faisceau $\mathfrak{w}_{I}^{\text {perf }}$ descend sur $\mathfrak{X}_{r, I}$ pour $r \geq 3$ (resp. $r \geq 5$ si $p=2$ ).

Le théorème 1.6 de [23] affirme que dans un espace rigide normal sur un corps, une fonction bornée sur un ouvert dense de Zariski se prolonge à tout l'espace. Il serait intéressant de savoir si cet énoncé reste valable pour un espace adique analytique. Le lemme suivant le montre dans un cas très particulier. 
Lemme 6.4. - On a un isomorphisme de faisceaux sur $\mathfrak{X}_{r, I}$ :

$$
\mathscr{O}_{\mathfrak{X}_{r, I}}=\lim _{k+1 \geq k^{\prime} \geq k \geq 1} \mathscr{O}_{\mathfrak{X}_{r,\left[p^{\mathrm{k}}, p^{k^{\prime}}\right]}} .
$$

Démonstration. - On vérifie d'abord $\mathscr{O}_{\left.\mathfrak{X}_{r,[p, p} k^{\prime \prime}\right]}=\lim _{k^{\prime \prime} \geq k+1 \geq k^{\prime} \geq k \geq 1} \mathscr{O}_{\mathfrak{X}_{r,\left[p^{\mathrm{k}}, p^{\left.k^{\prime}\right]}\right]}}$. Par la propriété de faisceau sur l'espace analytique ${ }^{e} \mathcal{C}_{r,\left[p, p^{k^{\prime \prime}}\right]}$, on a

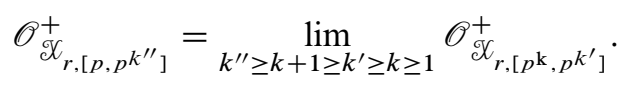

Notre première assertion résulte de la normalité des schémas formels $\mathfrak{X}_{r,\left[p^{\mathrm{k}}, p^{\left.k^{\prime}\right]}\right.}$. On se ramène donc à vérifier que $\mathscr{O}_{\mathfrak{X}_{r, I}}=\bigcap_{k \geq 0} \mathscr{O}_{\left.\mathfrak{X}_{r,[p, p}\right]}$. Il suffit de le vérifier sur une base d'ouverts de $\mathfrak{X}_{r, I}$. Soit $\operatorname{Spf} A$ un ouvert affine de $\mathfrak{X}$. On suppose que $\omega_{E}$ est trivial sur $\operatorname{Spf} A$. La fibre dans $\mathfrak{X}_{r, I}$ de $\operatorname{Spf} A$ vaut $\operatorname{Spf} B$ avec $B=A[[T]]\langle u, w\rangle /\left(w \tilde{\mathrm{Ha}}^{p^{r+1}}-T, T^{p} u-p\right)$ où Ha est un relèvement de l'invariant de Hasse identifié à un scalaire (voir la proposition 3.1). La fibre dans $\mathfrak{X}_{r,\left[p, p^{k}\right]}$ vaut $\operatorname{Spf} B_{k}$ avec $B_{k}=B\left\langle v_{k}\right\rangle /\left(u v_{k}-T^{p^{k}-p}\right)$. L'application $B_{k+1} \rightarrow B_{k}$ envoie $v_{k+1}$ sur $T^{p^{k+1}-p^{k}} v_{k}$. Soit $I_{k}=\left(w \tilde{\mathrm{Ha}}{ }^{p^{r+1}}-T, T^{p} u-p, u v_{k}-T^{p^{k}-p}\right)$ le noyau de la surjection $A[[T]]\left\langle u, w, v_{k}\right\rangle \rightarrow B_{k}$. Les suites exactes $0 \rightarrow I_{k} \rightarrow A[[T]]\left\langle u, w, v_{k}\right\rangle \rightarrow B_{k} \rightarrow 0$ forment un système projectif où les applications de transition sont celles qui envoient $v_{k+1}$ sur $T^{p^{k+1}-p^{k}} v_{k}$. En réduisant modulo $T^{n}$, on obtient un système projectif $0 \rightarrow$ $I_{k} / T^{n} \rightarrow A[[T]]\left\langle u, w, v_{k}\right\rangle / T^{n} \rightarrow B_{k} / T^{n} \rightarrow 0$ de suites exactes qui satisfait la condition de Mittag-Leffler. Il en résulte que $\lim _{k} B_{k} / T^{n}=B / T^{n}$. Puis, en passant à la limite sur $n$, on obtient $\lim _{n} \lim _{k} B_{k} / T^{n}=B$. Comme $\lim _{n} \lim _{k} B_{k} / T^{n}=\lim _{k} \lim _{n} B_{k} / T^{n}=\lim _{k} B_{k}$, on conclut.

Lemme 6.5. - On a un isomorphisme de faisceaux sur $\mathfrak{X}_{\infty, I}$ :

$$
\mathscr{O}_{\mathfrak{X}_{\infty, I}}=\lim _{k+1 \geq k^{\prime} \geq k \geq 1} \mathscr{O}_{\mathfrak{X}_{\infty,\left[p^{\mathrm{k}}, p^{k^{\prime}}\right]}} .
$$

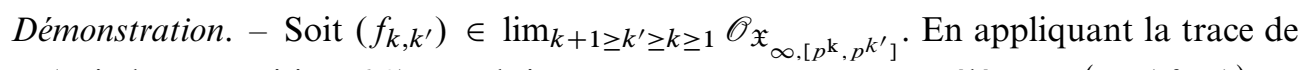
Tate (voir la proposition 6.2) on obtient, pour tout $r \geq 1$, un élément $\left(\operatorname{Tr}_{r}\left(f_{k, k^{\prime}}\right)\right) \in$ $\lim _{k+1 \geq k^{\prime} \geq k \geq 1} T^{-1} \mathscr{O}_{\mathfrak{X}_{r,\left[p^{\mathrm{k}}, p^{\left.k^{\prime}\right]}\right.}}$. D'après le lemme précédent, cet élément est représenté par un élément $f_{r} \in T^{-1} \mathscr{O}_{\mathfrak{X}_{r, I}}$. Pour $r$ assez grand, $\operatorname{Tr}_{r}\left(f_{1,1}\right) \in \mathscr{O}_{\mathfrak{X}_{\infty,\{p\}}}$ (c'est-à-dire qu'il n'y a pas de pôle en $T$ ). Ceci entraîne facilement que $f_{r} \in \mathscr{O}_{\mathfrak{x}_{r, I}}$. De même, on vérifie sans problème que $T \mathscr{O}_{\mathfrak{X}_{r,\{p\}}} \cap \mathscr{O}_{\mathfrak{X}_{r, I}}=T \mathscr{O}_{\mathfrak{X}_{r, I}}$. La convergence de $\operatorname{Tr}_{r}\left(f_{k, k^{\prime}}\right)$ vers $f_{k, k^{\prime}}$ entraîne donc la convergence de $f_{r}$ vers un élément $f \in \mathscr{O}_{\mathfrak{X}_{\infty, I}}$. Comme la restriction de $f$ à $\mathscr{O}_{\mathfrak{X}}{ }_{\infty,\left[p^{\mathrm{k}}, p^{\left.k^{\prime}\right]}\right.}$ vaut $f_{k, k^{\prime}}, f$ représente l'élément $\left(f_{k, k^{\prime}}\right)$.

Lemme 6.6. - Soit $r=3$ si $p \neq 2$ et $r=5$ si $p=2$. Soit $k \geq 1$. Supposons $r \leq n \leq k+r$. On $a$

$$
\kappa\left(1+p^{n-1} \mathbb{Z}_{p}\right)-1 \subset \operatorname{Hdg}_{r}^{\frac{p^{\mathrm{n}}-p}{p-1}} T^{2} \mathscr{O}_{\mathfrak{X}_{r,\left[p^{k}, \infty\right]}} .
$$

Démonstration. - Soit Spf $A$ un ouvert de $\mathfrak{X}_{r,\left[p^{k}, \infty\right]}$ sur lequel $\operatorname{Hdg}_{r}=(\tilde{\mathrm{Ha}} r)$. On a $\kappa\left(1+p^{n-1} \mathbb{Z}_{p}\right)-1 \subset\left(p T, T^{p^{n-2}}\right)\left(\right.$ resp. $\left(p T, T^{p^{n-3}}\right)$ pour $\left.p=2\right)$. On a alors dans $A$ les formules

$$
\frac{p}{\tilde{\mathrm{Ha}}_{r}^{p^{r+k}}}=p T^{-p^{k}}\left(\frac{T}{\tilde{\mathrm{Ha}}_{r}^{p^{r+1}}}\right)^{p^{k-1}} T^{p^{k}-p^{k-1}} \text { et } \frac{T^{p^{n-2}}}{\tilde{\mathrm{Ha}}_{r}^{p^{n+1}}}=\left(\frac{T}{\tilde{\mathrm{Ha}}_{r}^{p^{r+1}}}\right)^{p^{n-r}} T^{p^{n-2}-p^{n-r}} \text {. }
$$


Le lemme en résulte car $p^{k}-p^{k-1}+1 \geq 2$ pour tout $k \geq 1, p^{n-2}-p^{n-3} \geq 2$ pour tout $n \geq 3$ si $p \neq 2$ et $p^{n-3}-p^{n-5} \geq 2$ si $p=2$ et $n \geq 5$.

Nous sommes maintenant préparés pour démontrer la descente.

THÉORÈME 6.4. - Le faisceau $\mathfrak{w}_{I}^{\text {perf }}$ descend en un faisceau inversible $\mathfrak{w}_{I}$ sur $\mathfrak{X}_{r, I}$ avec $r \geq 3$ si $p \geq 3$ et $r \geq 5$ si $p=2$. Précisément, $\mathfrak{w}_{I}$ est l'unique sous-faisceau cohérent de $\mathscr{O}_{\mathfrak{X}_{r, I}}$-modules de $\mathfrak{w}_{I}^{\text {perf }}$ tel que, pour tout intervalle $J \subset I$, si on note $i_{J, I}: \mathfrak{X}_{r, J} \rightarrow \mathfrak{X}_{r, I}$ l'application évidente, alors on a un isomorphisme canonique $i_{J, I}^{\star} \mathfrak{w}_{I}=\mathfrak{w}_{J}$ induit par l'isomorphisme $i_{J, I}^{\star} \mathfrak{w}_{I}^{\text {perf }}=\mathfrak{w}_{J}^{\text {perf }}$.

Démonstration. - On pose $\mathfrak{w}_{I}=\lim _{k+1 \geq k^{\prime} \geq k \geq 1} \mathfrak{w}_{\left[p^{\mathrm{k}}, p^{\left.k^{\prime}\right]}\right.}$. C'est un faisceau de $\mathscr{O}_{\mathfrak{x}_{r, I}}$-modules. Nous allons commencer par démontrer que c'est un faisceau inversible qui descend le faisceau $\mathfrak{w}_{\mathrm{I}}^{\text {perf }}$. La définition de $\mathfrak{w}_{I}$ est motivée par le numéro 5.1.3. Il suffit de démontrer le théorème lorsque $r$ est le plus petit possible. Nous supposons donc que $r=5$ si $p=2$ et $r=3$ si $p \geq 3$. Commençons par fixer un ouvert $\operatorname{Spf} A$ de $\mathfrak{X}$ sur lequel le faisceau $\omega_{\mathrm{E}}$ est trivial. Soit Spf $B$ l'image inverse de cet ouvert dans $\mathfrak{X}_{r, I}$. Comme $\omega_{\mathrm{E}}$ est trivial, $\operatorname{Hdg}_{r}$ est trivial, engendré par un élément $\tilde{\mathrm{Ha}}{ }_{r}$. Soient

$$
\begin{aligned}
c_{0} & =1 \in \mathscr{O}_{\mathfrak{I} \mathfrak{G}_{0, r, I}}(\operatorname{Spf} B), & & \\
c_{1} & =\frac{1}{p-1} \in \mathscr{O}_{\mathfrak{I} \mathfrak{G}_{1, r, I}}(\operatorname{Spf} B), & & \\
c_{n} & \in \tilde{\mathrm{Ha}}_{r}^{-\frac{p^{\mathrm{n}}-p}{p-1}} \mathscr{O}_{\mathfrak{I} \mathfrak{G}_{n, r}, I}(\operatorname{Spf} B), & & \text { pour } n \leq r \text { et } \\
c_{n} & \in \tilde{\mathrm{Ha}}_{r}^{-p^{\mathrm{r}}} \mathscr{O}_{\mathfrak{I G}_{n, \infty, I}}(\operatorname{Spf} B) & & \text { pour } n \geq r+1,
\end{aligned}
$$

des éléments tels que $\operatorname{Tr}_{\mathfrak{I G}}\left(c_{n}\right)=c_{n-1}$ (ces élements existent d'après le corollaire 3.1 et la proposition 6.1). On pose $b_{n}=\sum_{\sigma \in\left(\mathbb{Z} / p^{n} \mathbb{Z}\right)^{\times}} \kappa(\tilde{\sigma}) \sigma \cdot c_{n}$, où $\tilde{\sigma}$ est un relèvement de $\sigma$ dans $\mathbb{Z}_{p}^{\times}$. On a $b_{n}-b_{n-1} \in T^{n-1} \tilde{\mathrm{Ha}}_{r}^{-p^{\mathrm{r}}}\left(\right.$ resp. $T^{n-2} \tilde{\mathrm{Ha}}_{r}^{-p^{\mathrm{r}}}$ si $\left.p=2\right)$. Soit $b_{\infty} \in \mathscr{O}_{\mathfrak{I G}_{\infty, \infty, I}}(\operatorname{Spf} B)$ la limite des $b_{n}$. Comme dans la démonstration de la proposition 6.4 , on voit que $b_{\infty}=1 \bmod T \tilde{\mathrm{Ha}}^{-p^{r}}$. C'est donc un générateur du faisceau $\mathfrak{w}_{I}^{\text {perf }}$ sur Spf $B$. On observe également que $b_{\infty}-b_{r}=1 \bmod T^{\mathrm{r}} \tilde{\mathrm{Ha}}_{r}^{-p^{\mathrm{r}}}$ et donc $b_{\infty}-b_{r}=1 \bmod T^{2}$.

Soit $J=\left[p^{k}, p^{k+1}\right]$ avec $k \geq 1$. Considérons le diagramme commutatif :

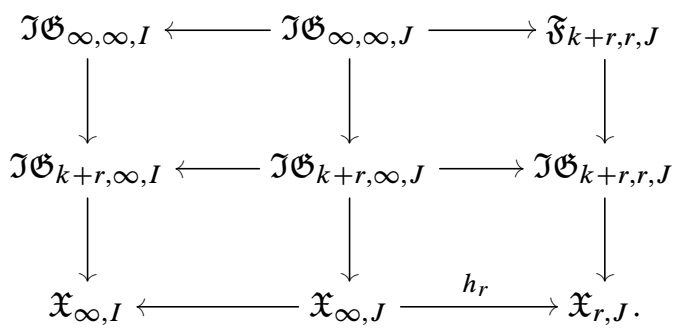

Soit Spf $C$ l'image inverse de $\operatorname{Spf} B$ dans $\mathfrak{X}_{r, J}$. Soit $s \in \mathscr{O}_{\mathfrak{F}_{k+r, r},}(\operatorname{Spf} C)$ une section telle que $\sigma . s=\kappa^{-1}(\sigma) s$ pour tout $\sigma \in\left(1+p^{k+r} \operatorname{Hdg}_{r}^{-\frac{p^{k+r}}{p-1}} \mathbb{G}_{a}\right)$ et $s=1 \bmod q($ voir le lemme 5.3). Pour tout $0 \leq n \leq r+k$, soit $c_{n}^{\prime} \in \tilde{\mathrm{Ha}}_{r}^{-\frac{p^{\mathrm{n}}-p}{p-1}} \mathscr{O}_{\mathfrak{I G}_{n, r, J}}(\operatorname{Spf} C)$ des éléments tels que $\operatorname{Tr}_{\mathfrak{I G}}\left(c_{n}^{\prime}\right)=c_{n-1}^{\prime}$ et $c_{n}^{\prime}=c_{n}$ si $n \leq r$. On note alors $f=\sum_{\sigma \in\left(\mathbb{Z} / p^{k+r} \mathbb{Z}\right)^{\times}} \kappa(\sigma) \sigma \cdot c_{r+k}^{\prime} s$. 
C'est un générateur de $\mathfrak{w}_{J}$ sur Spf $C$, d'après le lemme 5.4. Comme $T^{p^{k}} \mid p$ et $\tilde{\mathrm{Ha}}_{r}{ }^{r+1} \mid T$ dans $C$, il en résulte que

$$
p \tilde{\mathrm{Ha}}_{r}^{-p^{r+k}}=\left(p T^{-p^{\mathrm{k}}}\right)\left(T \tilde{\mathrm{Ha}}_{r}^{-p^{r+1}}\right)^{p^{k-1}} T^{p^{k}-p^{k-1}} .
$$

Comme $f=\sum_{\sigma \in\left(\mathbb{Z} / p^{k+r} \mathbb{Z}\right)^{\times}} \kappa(\tilde{\sigma}) \sigma \cdot c_{r+k}^{\prime} \bmod q \tilde{\mathrm{Ha}}_{r}^{-p^{r+k}}, \quad$ on obtient donc que $f=\sum_{\sigma \in\left(\mathbb{Z} / p^{k+r} \mathbb{Z}\right)^{\times}} \kappa(\tilde{\sigma}) \sigma \cdot c_{r+k}^{\prime} \bmod T^{2}$. Montrons maintenant par récurrence descendante sur $r \leq n \leq r+k$ que $f=\sum_{\sigma \in\left(\mathbb{Z} / p^{\mathrm{n}} \mathbb{Z}\right)^{\times}} \kappa(\tilde{\sigma}) \sigma \cdot c_{\mathrm{n}}^{\prime} \bmod T^{2}$.

$$
\begin{aligned}
\sum_{\sigma \in\left(\mathbb{Z} / p^{\mathrm{n}} \mathbb{Z}\right)^{\times}} \kappa(\tilde{\sigma}) \sigma \cdot c_{\mathrm{n}}^{\prime} & =\sum_{\tau \in\left(\mathbb{Z} / p^{n-1} \mathbb{Z}\right)^{\times}} \kappa(\tilde{\tau}) \tilde{\tau} .\left(\sum_{\sigma \in\left(1+p^{n-1} \mathbb{Z} / p^{\mathrm{n}} \mathbb{Z}\right)} \kappa(\tilde{\sigma}) \sigma \cdot c_{\mathrm{n}}^{\prime}\right) \\
& =\sum_{\tau \in\left(\mathbb{Z} / p^{n-1} \mathbb{Z}\right)^{\times}} \kappa(\tilde{\tau}) \tilde{\tau} .\left(c_{n-1}^{\prime}+\sum_{\sigma \in\left(1+p^{n-1} \mathbb{Z} / p^{\mathrm{n}} \mathbb{Z}\right)}(\kappa(\tilde{\sigma})-1) \sigma \cdot c_{\mathrm{n}}^{\prime}\right) .
\end{aligned}
$$

D'après le lemme 6.6, $(\kappa(\tilde{\sigma})-1) \sigma \cdot c_{\mathrm{n}}^{\prime}=0 \bmod T^{2}$. On conclut donc la récurrence. Vérifions à présent que $f=b_{\infty} \bmod T^{2}$ vues comme des sections de $\mathscr{O}_{\mathfrak{I} \mathfrak{G}_{\infty, \infty}, J}(\operatorname{Spf} C)$.

On a alors dans $\mathscr{O}_{\mathfrak{I G} \infty, \infty, J}(\operatorname{Spf} C) \bmod T^{2}$ :

$$
\begin{aligned}
f & =\sum_{\sigma \in\left(\mathbb{Z} / p^{k+r} \mathbb{Z}\right)^{\times}} \kappa(\tilde{\sigma}) \sigma \cdot c_{r+k}^{\prime} \\
& =\sum_{\sigma \in \mathbb{Z} / p^{r} \mathbb{Z}} \kappa(\tilde{\sigma}) \sigma \cdot c_{r} \\
& =b_{r} \\
& =b_{\infty} .
\end{aligned}
$$

On a donc $b_{\infty}=\left(1+T^{2} u\right) f$ pour une fonction $u \in \mathscr{O}_{\mathfrak{X}_{\infty, J}}(\operatorname{Spf} C)$. Il en résulte que $\operatorname{Tr}_{r}\left(b_{\infty}\right)=f\left(1+T^{2} \operatorname{Tr}_{r}(u)\right)$. Comme $\operatorname{Tr}_{r}(u) \in T^{-1} C$ d'après la proposition 6.2, il en résulte que $\operatorname{Tr}_{r}\left(b_{\infty}\right)$ est un générateur de $\mathfrak{w}_{J}$ sur $\operatorname{Spf} C$. Comme la construction de $\operatorname{Tr}_{r}\left(b_{\infty}\right)$ est fonctorielle en $J$, on a $\mathfrak{w}_{I}(\operatorname{Spf} B)=\operatorname{Tr}_{r}\left(b_{\infty}\right) \lim _{k+1 \geq k^{\prime} \geq k \geq 1} \mathscr{O}_{\mathfrak{X}_{r,\left[p^{k}, p^{\left.k^{\prime}\right]}\right.}}(\operatorname{Spf} B)=\operatorname{Tr}_{r}\left(b_{\infty}\right) \cdot B$ d'après le lemme 6.4. Ceci démontre que le faisceau $\mathfrak{w}_{I}$ est un faisceau inversible de $\mathscr{O}_{\mathfrak{x}_{r, I}}$-modules et que pour tout intervalle $J \subset I, i_{J, I}^{\star} \mathfrak{w}_{I}=\mathfrak{w}_{J}$. D'autre part, le lemme 6.5 entraîne que $\lim _{k+1 \geq k^{\prime} \geq k \geq 1} \mathfrak{w}_{\left[p^{k}, p^{\left.k^{\prime}\right]}\right.}^{\text {perf }}=\mathfrak{w}_{I}^{\text {perf }}$. Ceci entraîne alors que $h_{r}^{\star} \mathfrak{w}_{I}=\mathfrak{w}_{I}^{\text {perf }}$. Le faisceau $\mathfrak{w}_{I}$ descend bien le faisceau $\mathfrak{w}_{I}^{\text {perf }}$. Démontrons maintenant l'unicité. Soit $\mathfrak{w}_{I}^{\prime} \subset \mathfrak{w}_{I}^{\text {perf }}$ un faisceau cohérent de $\mathscr{O}_{\mathfrak{X}_{r, I}}$-modules ayant les propriétés du théorème. Il est clair que l'inclusion $\mathfrak{w}_{I}^{\prime} \subset \mathfrak{w}_{I}^{\text {perf }}$ se factorise à travers $\mathfrak{w}_{I}^{\prime} \hookrightarrow \mathfrak{w}_{I}$. Cette application induit pour tout $k+1 \geq k^{\prime} \geq k \geq 1$ un isomorphisme $\mathfrak{w}_{I}^{\prime} \otimes \mathscr{O}_{\mathfrak{X}_{r,\left[p^{k}, p^{\left.k^{\prime}\right]}\right.}} \rightarrow \mathfrak{w}_{I} \otimes \mathscr{O}_{\mathfrak{X}_{r,[p}, p^{\left.k^{\prime}\right]}}$ et on conclut par le lemme 6.4.

Remarque 6.2. - La démonstration montre plus précisément que le faisceau $\mathfrak{w}_{I}$ est trivial sur l'image inverse de tout ouvert affine de $\mathfrak{X}$ au-dessus duquel le faisceau $\omega_{E}$ est trivial.

Proposition 6.7. - On a un opérateur de Frobenius :

$$
i^{\star} \mathfrak{w}_{[p, \infty]} \simeq \phi^{\star} \mathfrak{w}_{[p, \infty]}
$$


où $i: \mathfrak{X}_{r+1,[p, \infty]} \rightarrow \mathfrak{X}_{r,[p, \infty]}$ est le morphisme d'inclusion et $\phi: \mathfrak{X}_{r+1,[p, \infty]} \rightarrow \mathfrak{X}_{r,[p, \infty]}$ est le Frobenius.

Démonstration. - Le faisceau $\mathfrak{w}_{I}$ est canoniquement déterminé à partir des faisceaux $\mathfrak{w}_{\mathrm{I}}^{\text {perf }}$ et $\mathfrak{w}_{J}$ pour $J \subset[p, \infty[$. Il hérite donc des fonctorialités de ces derniers.

\subsection{Comparaison avec le faisceau $\mathfrak{w}_{\{\infty\}}$}

Dans la section précédente, nous avons construit un faisceau $\mathfrak{w}_{\text {I }}$ sur l'intervalle $I=[p, \infty]$. Nous allons maintenant vérifier que sa fibre en l'infini est bien le faisceau $\mathfrak{w}_{\{\infty\}}$ du numéro 4.4.1.

Proposition 6.8. - Pour tout entier $k_{0} \geq 1$, l'inclusion naturelle de $\mathfrak{w}_{\left[p^{k_{0}}, \infty\right]}$ dans $\mathscr{O}_{\mathfrak{I G}}{ }_{\infty, \infty,\left[p^{\left.k_{0}, \infty\right]}\right.}$ se factorise modulo $T^{p^{k_{0}}-p^{k_{0}-1}-1}$ en un morphisme

$$
\mathfrak{w}_{\left[p^{\left.k_{0}, \infty\right]}\right.} \rightarrow \mathscr{O}_{\mathfrak{I G}}{ }_{r+k_{0}, r,\left[p^{\left.k_{0}, \infty\right]}\right.} / T^{p^{k_{0}-p^{k_{0}-1}-1}} .
$$

La restriction de $\mathfrak{w}_{I}$ à $\mathfrak{X}_{r,\{\infty\}}$ est un sous-faisceau de $\mathscr{O}_{\mathfrak{I} \mathfrak{G}_{\infty, r,\{\infty\}}}$ qui s'identifie canoniquement $\grave{a} \mathfrak{w}_{\{\infty\}}$.

Démonstration. - Fixons un entier $k_{0}$. Soit Spf $B$ un ouvert affine de $\mathfrak{X}_{r,\left[p^{\left.k_{0}, \infty\right]}\right.}$. On suppose $\operatorname{Hdg}$ trivial sur $\operatorname{Spf} B$, engendré par un élément H̃a. Fixons des éléments $c_{0}=1$ et, pour $1 \leq n \leq k_{0}+r, c_{n} \in \tilde{\mathrm{Ha}}^{-\frac{p^{n}-p}{p-1}} \mathscr{O}_{\mathfrak{I} \mathfrak{G}_{n, r,\left[p^{k}, \infty\right]}}(\operatorname{Spf} B)$ tels que $\operatorname{Tr}_{\mathfrak{I G}}\left(c_{n}\right)=c_{n-1}$. Complétons cette suite en choisissant, pour $n \geq r+k_{0}+1$, des éléments $c_{n} \in \tilde{\mathrm{Ha}}^{-p^{r+k_{0}}} \mathscr{O}_{\mathfrak{I G}{ }_{n, \infty,[p} k_{0, \infty]}}(\operatorname{Spf} B) \quad$ vérifiant $\quad \operatorname{Tr}_{\mathfrak{I G}}\left(c_{n}\right)=c_{n-1} . \quad$ On pose $b_{n}=\sum_{\sigma \in\left(\mathbb{Z} / p^{n} \mathbb{Z}\right)^{\times}} \kappa(\tilde{\sigma}) \sigma . c_{n}$, où $\tilde{\sigma}$ est un relèvement de $\sigma$ dans $\mathbb{Z}_{p}^{\times}$. La suite $b_{n}$ converge dans $\mathscr{O}_{\mathfrak{I G}}{ }_{\infty, \infty,\left[p^{\left.k_{0}, \infty\right]}\right.}$ vers un générateur $b_{\infty}$ du faisceau $\mathfrak{w}_{\mathrm{I}}^{\text {perf }}$ (d'après la démonstration de la proposition 6.4). On vérifie que pour tout $n \geq r+k_{0}, b_{n+1}=b_{\mathrm{n}} \bmod T^{p^{k_{0}}-p^{k_{0}}-1}$. En effet, en raisonnant comme dans la démonstration du théorème 4.1, on voit que

$$
b_{n+1}=b_{n} \bmod \left(\kappa\left(1+p^{r+k_{0}}\right)-1\right) \tilde{\mathrm{Ha}}^{-p^{r+k_{0}}}
$$

or on observe que $\left.\left(\kappa\left(1+p^{r+k_{0}}\right)-1\right)\right) \subset\left(T^{p^{k_{0}}}\right)$ et $T^{p^{k_{0}-1}} \in\left(\tilde{\mathrm{Ha}}{ }^{-p^{r+k_{0}}}\right)$. Il en résulte que $b_{\infty}=b_{r+k_{0}} \bmod T^{p^{k_{0}}-p^{k_{0}-1}}$.

Fixons à présent un intervalle $\left[p^{\mathrm{k}}, p^{k+1}\right]$ avec $k \geq k_{0}$. Soit Spf $C$ l'image inverse de Spf $B$ dans $\mathfrak{X}_{r,\left[p^{\mathrm{k}}, p^{k+1}\right]}$. Fixons des élements $c_{\mathrm{n}}^{\prime} \in \tilde{\mathrm{Ha}}{ }^{-\frac{p^{n}-p}{p-1}} \mathscr{O}_{\mathfrak{I} \mathfrak{G}_{n, r,\left[p^{\mathrm{k}}, p^{k+1}\right]}}(\operatorname{Spf} C)$ pour $r+k_{0}+1 \leq n \leq r+k$ vérifiant $\operatorname{Tr}_{\mathfrak{I} \mathfrak{G}}\left(c_{n}^{\prime}\right)=c_{n-1}^{\prime}$ pour $n \geq r+k_{0}+2$ et $\operatorname{Tr}_{\mathfrak{I G}}\left(c_{r+k_{0}+1}^{\prime}\right)=c_{r+k_{0}}$. Il existe alors un générateur $f$ du faisceau $\mathfrak{w}_{I}$ sur Spf $C$ tel que $f=\sum_{\sigma \in\left(\mathbb{Z} / p^{r+k} \mathbb{Z}\right)^{\times}} \kappa(\tilde{\sigma}) \sigma \cdot c_{r+k}^{\prime} \bmod p \tilde{H a}^{-p^{r+k}}$ d'après le lemme 5.4. Dans $C, T^{p^{k}} \mid p$ et $\tilde{\mathrm{Ha}}^{-p^{r+k}} \mid T^{p^{k-1}}$. Ceci entraîne que $f=\sum_{\sigma \in\left(\mathbb{Z} / p^{r+k} \mathbb{Z}\right)^{\times}} \kappa(\tilde{\sigma}) \sigma \cdot c_{r+k}^{\prime} \bmod T^{p^{k_{0}}-p^{k_{0}-1}}$. En raisonnant comme au-dessus on obtient alors $f=b_{r+k_{0}} \bmod T^{p^{k_{0}}-p^{k_{0}-1}}$. Par conséquent, dans $\mathscr{O}_{\mathfrak{I G}}{ }_{\infty, \infty,\left[p^{k}, p^{k+1}\right]}(\operatorname{Spf} C)$ on a $f=b_{\infty}=b_{r+k_{0}} \bmod T^{p^{k_{0}}-p^{k_{0}-1}}$. En appliquant la trace de Tate on obtient $f=\operatorname{Tr}_{r}\left(b_{\infty}\right)=b_{\infty} \bmod T^{p^{k}-p^{k}-1}-1 \mathfrak{w}_{\left[p^{k}, p^{k+1}\right]}^{\text {perf }}(\operatorname{Spf} C)$. Comme cette relation est valable sur les intervalles $\left[p^{k}, p^{k+1}\right]$, elle entraîne que 
$\operatorname{Tr}_{\mathrm{r}}\left(b_{\infty}\right)=b_{\infty} \bmod T^{p^{k_{0}}-p^{k_{0}-1}-1} \mathfrak{w}_{\left[p^{\left.k_{0}, \infty\right]}\right.}^{\text {perf }}(\operatorname{Spf} B)$ par une variante évidente du lemme 6.5. Il en résulte que $\operatorname{Tr}_{\mathrm{r}}\left(b_{\infty}\right)=b_{r+k_{0}} \bmod T^{p^{k_{0}}-p^{k_{0}-1}-1} \mathscr{O}_{\mathfrak{I G}}{ }_{\infty, \infty,\left[p^{\left.k_{0}, \infty\right]}\right.}(\operatorname{Spf} B)$.

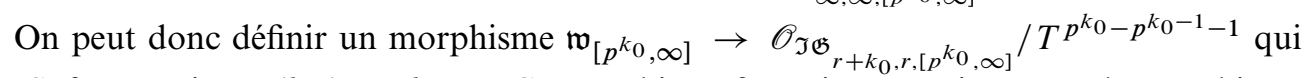
sur Spf $B$ envoie $\operatorname{Tr}_{r}\left(b_{\infty}\right)$ sur $b_{r+k_{0}}$. Ce morphisme factorise canoniquement le morphisme $\mathfrak{w}_{\left[p^{\left.k_{0}, \infty\right]}\right.} \rightarrow \mathscr{O}_{\mathfrak{I G}}{ }_{\infty, \infty,\left[p^{\left.k_{0}, \infty\right]}\right.} / T^{p^{k_{0}}-p^{k_{0}-1}-1}$. En comparant la définition de $b_{r+k_{0}}$ et la construction du faisceau $\mathfrak{w}_{\{\infty\}}$ (voir le théorème 4.1), on obtient que la restriction de $\mathfrak{w}_{\mathrm{I}}$ à $\mathfrak{X}_{r,\{\infty\}}$ vaut $\mathfrak{w}_{\{\infty\}}$.

\subsection{La partie finie du caractère}

Rappelons que $\mathfrak{W}_{I}=\operatorname{Spf} B_{I}\left[(\mathbb{Z} / q \mathbb{Z})^{\times}\right]$et que $\mathfrak{M}_{r, I}=\mathfrak{X}_{r, I} \otimes_{\mathfrak{W}_{I}^{0}} \mathfrak{W}_{I}$ (voir 3.3 pour les notations). Soit $\kappa^{f}:(\mathbb{Z} / q \mathbb{Z})^{\times} \rightarrow B_{I}\left[(\mathbb{Z} / q \mathbb{Z})^{\times}\right]$le caractère universel. Soit $h: \mathfrak{I G}_{i, r, I} \times_{\mathfrak{W}_{I}^{0}} \mathfrak{W}_{I} \rightarrow \mathfrak{M}_{r, I}$ le morphisme structural où $i=1$ si $p \neq 2$ et $i=2$ si $p=2$. On

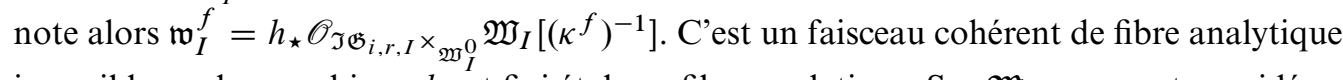
inversible car le morphisme $h$ est fini étale en fibre analytique. Sur $\mathfrak{M}_{r, I}$ on peut considérer le faisceau $\mathfrak{w}_{I} \otimes \mathfrak{w}_{I}^{f}$. Sa fibre analytique sur $\mathcal{M}_{r, I}$ est le faisceau $\omega_{I}^{\kappa}$.

\subsection{Construction de la courbe de Hecke adique}

Dans ce numéro, nous démontrons le théorème 6.3.

6.9.1. Projectivité. - Dans cette sous-partie nous posons $I=[p, \infty]$. Fixons $r \geq 3$ (resp. $r \geq 5$ si $p=2)$. Soit $M=\mathrm{H}^{0}\left(\mathcal{M}_{r, I}, \omega_{I}^{\kappa}\right)$. Pour alléger les notations, posons $B_{0}=B_{I}\left[\mathbb{Z} / q \mathbb{Z}^{\times}\right]$et $B=B_{0}[1 / T]$.

Proposition 6.9. - Le B-module de Banach M est projectif.

Démonstration. - Fixons un recouvrement affine fini $\bigcup_{i} \operatorname{Spf} A_{i}$ de la courbe modulaire $\mathfrak{X}$ sur Spf $\mathbb{Z}_{p}$, tel que sur chaque ouvert $\operatorname{Spf} A_{i}$, le faisceau $\omega_{E}$ est trivial. Soit $V_{i}$ l'image inverse de $\operatorname{Spf} A_{i}$ dans $\mathcal{M}_{r, I}$. Notons que $\bigcup_{i} V_{i}$ est un recouvrement affinoïde de l'affinoïde $\mathcal{M}_{r, I}$. D'après [19], Thm. 2.2, on a une suite exacte longue

$$
0 \rightarrow M \rightarrow \oplus_{i} \mathrm{H}^{0}\left(V_{i}, \omega_{I}^{\kappa}\right) \rightarrow \oplus_{i \neq j} \mathrm{H}^{0}\left(V_{i} \cap V_{j}, \omega_{I}^{\kappa}\right) \cdots
$$

et on est donc ramené à montrer la projectivité de $\mathrm{H}^{0}\left(V, \omega_{I}^{\kappa}\right)$ où $V$ est l'intersection de certains ouverts $V_{i}$ (corollaire B.2). Soit Spf $A$ une intersection de certains $\operatorname{Spf} A_{i}$ et soit $\operatorname{Spf} C$ l'image inverse de $\operatorname{Spf} A$ dans $\mathfrak{X}_{r, I}$. Alors par la remarque 6.2, le faisceau $\mathfrak{w}_{\mathrm{I}}(\operatorname{Spf} C)$ est trivial. Vérifions que Spf $C$ est la complétion d'un $B_{I}$-module libre. Par construction,

$$
B_{I}=\mathbb{Z}_{p}[[T]]\langle u\rangle /\left(T^{p} u-p\right) \text { et } C \simeq A[[T]]\langle u, v\rangle /\left(T^{p} u-p, \tilde{\mathrm{Ha}}^{p^{r+1}} v-T\right) .
$$

Il en résulte que $B_{I} / T B_{I}=\mathbb{F}_{p}[u]$ et $C / T C=(A / p)[v, u] /\left(\tilde{\mathrm{Ha}}{ }^{p+1} v-T\right)$. Soit $\left(e_{j}\right)_{j \in J}$ une base algébrique du $\mathbb{F}_{p}$-espace vectoriel $(A / p)[v] /\left(\tilde{\mathrm{Ha}}^{p r+1} v-T\right)$. Un relèvement arbitraire de cette base dans $C$ fournit une base topologique de $C$ comme $B_{I}$-module.

Soit Spf $D$ l'image inverse de Spf $C$ dans la tour d'Igusa partielle $\mathfrak{I G}_{i, r, I}$ avec $i=1$ si $p \neq 2$ et $i=2$ si $p=2$. Alors $D[1 / T]$ est une algèbre finie étale sur $C[1 / T]$ et en particulier, $D[1 / T]$ est un $B_{I}[1 / T]$ module de Banach projectif. Par conséquent, 
$D\left[(\mathbb{Z} / q \mathbb{Z})^{\times}\right][1 / T]$ est un $B$-module projectif. Soit $\kappa^{f}:(\mathbb{Z} / q \mathbb{Z})^{\times} \rightarrow B^{\times}$le caractère tautologique. Soit $D\left[(\mathbb{Z} / q \mathbb{Z})^{\times}\right][1 / T]\left[\left(\kappa^{f}\right)^{-1}\right]$ le facteur direct de $D\left[(\mathbb{Z} / q \mathbb{Z})^{\times}\right][1 / T]$ des éléments qui se transforment via $\kappa^{f}$ pour l'action de $(\mathbb{Z} / q \mathbb{Z})^{\times}$. C'est donc un $B$-module projectif. Par définition, ce $B$-module est $\mathrm{H}^{0}\left(V, \omega_{\mathrm{I}}^{\kappa}\right)$.

6.9.2. La courbe de Hecke. - Soit $f_{r}: M_{r,[0, \infty]} \rightarrow W$ le morphisme structural. Soit $\Omega_{r}=\left(f_{r}\right)_{\star} \omega_{[0, \infty]}^{\kappa}$ le faisceau des formes $r$-surconvergentes paramétrées par $W$.

Lemme 6.7. - Pour tout ouvert affine $\mathscr{W}_{I} \subset \mathscr{W}_{,} \Omega_{r}\left(\mathscr{W}_{I}\right)$ est un $\mathscr{O}_{\omega_{I}}$-module projectif.

Démonstration. - Sur un ouvert de l'espace $\mathscr{W}_{[0, \infty[}$, c'est démontré dans [25], sect. 5.2. Pour un ouvert inclus dans $\mathscr{W}_{[p, \infty]}$, cela résulte de la proposition 6.9.

On définit sur $\Omega_{r}$ une action de l'opérateur $U_{p}$ par la règle suivante (pour tout intervalle $I \subset[0, \infty]):$

$$
U_{p}: \mathrm{H}^{0}\left(\mathcal{M}_{r, I}, \omega_{I}^{\kappa}\right) \rightarrow \mathrm{H}^{0}\left(\mathcal{M}_{r+1, I}, i^{\star} \omega_{I}^{\kappa}\right) \simeq \mathrm{H}^{0}\left(\mathcal{M}_{r+1, I}, \phi^{\star} \omega_{I}^{\kappa}\right) \stackrel{p^{-1} \operatorname{Tr}_{\phi}}{\rightarrow} \mathrm{H}^{0}\left(\mathcal{M}_{r, I}, \omega_{I}^{\kappa}\right)
$$

Cet opérateur est compact. On peut donc définir la série caractéristique $\mathscr{P}$ de $U_{p}$ agissant sur $\Omega_{r}$. Cette série caractéristique est à coefficients dans $\mathrm{H}^{0}\left(\mathscr{W}_{,} \mathscr{O}_{\omega}\right)=\Lambda$.

REMARQue 6.3. - On retrouve là le coro. 12.1 de [10] par une autre manière.

De plus, la formation de $\mathscr{P}$ commute au changement de base sur $\mathscr{W}$. En particulier, si $\left\{\bar{\kappa}_{\chi}\right\} \hookrightarrow \mathscr{W}$ est un point du bord, alors $\mathscr{P}_{\left.\right|_{\left\{\bar{\kappa}_{\chi}\right\}}}$ est la série caractéristique de $U_{p}$ agissant $\operatorname{sur} M_{\bar{\kappa}_{\chi}}^{\dagger}$.

L'action de l'algèbre de Hecke $H$ de niveau premier à $N p$ se définit comme dans le cas usuel. En appliquant la construction B.3, on obtient une variété spectrale $\mathscr{Z} \rightarrow \mathscr{W}$ et une courbe de Hecke $\mathcal{E} \rightarrow \mathscr{Z} \rightarrow$ ).

Proposition 6.10. - Soit $f \in M_{\bar{\kappa}_{\chi}}^{\dagger}$ une forme propre surconvergente de pente finie. Soit $x_{f} \in \mathcal{E}$ le point associé. Il existe un voisinage $\mathscr{W}_{\bar{\kappa}_{\chi}}$ de $\bar{\kappa}_{\chi}$ dans $W_{\text {et }}$ un voisinage $\mathcal{E}_{x_{f}}$ de $x_{f}$ dans $\mathcal{E}$ tel que le morphisme associé $\mathcal{E}_{x_{f}} \rightarrow \mathcal{W}_{\bar{\kappa}_{\chi}}$ soit fini et sans torsion.

De plus, pour tout entier $k \geq 2$, on peut trouver une suite $\left(f_{n}\right)$ de formes propres classiques de pente finie et de poids $k$, de point associé $x_{f_{n}}$ sur $\mathcal{E}_{x_{f}}$, tel que $x_{f_{n}} \rightarrow x_{f}$ quand $n \rightarrow \infty$.

Démonstration. - L'existence d'un morphisme $\mathcal{E}_{x_{f}} \rightarrow \mathscr{W}_{\bar{\kappa}_{\chi}}$ ayant les propriétés annoncées résulte de la proposition B.4. Voyons $k \in \mathbb{Z}$ comme un caractère algébrique de $\mathbb{Z}_{p}^{\times}$. Soit $\left\{\chi_{n}: \mathbb{Z}_{p}^{\times} \rightarrow \mathbb{C}_{p}^{\times}\right\}$une suite de caractères finis de conducteur $p^{n}$. On suppose de plus que $\left.\chi_{n}\right|_{\mathbb{Z} / p \mathbb{Z}^{\times}}=\chi k^{-1}$ est indépendant de $n$. On vérifie facilement que la suite de carac-

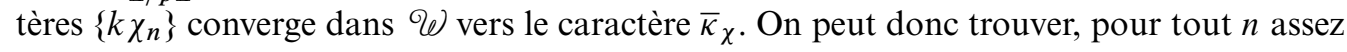
grand, une suite de points $x_{n} \in \mathcal{E}_{x_{f}}$ de poids $k \chi_{n}$ tel que $\lim _{n \rightarrow \infty} x_{n}=x$. On note $v_{x_{n}, T}$ la valuation correspondant aux points $x_{n}$, normalisée par $v_{x_{n}, T}(T)=1$. On note $v_{x_{n}, p}$ la valuation correspondant aux points $x_{n}$ normalisée par $v_{x_{n}, p}(p)=1$. On note $\alpha$ l'image de $U_{p}$ dans $\mathscr{O}_{\mathcal{E}_{x_{f}}}$. Pour $n$ assez grand, la pente $T$-adique de $x_{n}$ (qui vaut $v_{x_{n}, T}(\alpha)$ ) est la pente $T$-adique de $f$ notée $\alpha_{f}$. Il en résulte que la pente $p$-adique de $x_{n}$, qui vaut $v_{x_{n}, p}(\alpha)=$ $v_{x_{n}, T}(p)^{-1} \alpha_{f}$, tend vers 0 quand $n$ tend vers l'infini. D'après [9], les points $x_{n}$ sont classiques pour $n$ assez grand. 


\section{Appendice A}

\section{Théorie du sous-groupe canonique}

La théorie du sous-groupe canonique a été considérée par de nombreux auteurs ([21], [1], [2], [14], [29] notamment). Le point de vue adopté est cependant toujours $p$-adique. Nous reformulons ici la théorie au niveau de généralité dont nous avons besoin, c'est-à-dire sans supposer que la topologie est $p$-adique. Nous allons reprendre la méthode expliquée dans [26], sect. III.2, qui repose sur la théorie du complexe cotangent d'Illusie.

\section{A.1. Invariant de Hasse et idéal de Hodge}

Si $H \rightarrow$ Spec $R$ est un schéma en groupes, on note $\ell_{H / R}$ son complexe de co-Lie et $\omega_{\mathrm{H}}=\mathrm{H}^{0}\left(\ell_{H / R}\right)$ son faisceau conormal ([24]). Supposons que $R$ est un anneau de caractéristique $p$. Soit $G$ un groupe de Barsotti-Tate tronqué sur $R$. On note $F$ et $V$ les morphismes de Frobenius et Verschiebung. Le complexe de co-Lie de Ker $V$ est représenté par :

$$
\left[\omega_{\mathrm{G}} \stackrel{H W(G)}{\longrightarrow} \omega_{G^{(p)}}\right]
$$

où $H W(G)$ s'appelle la matrice de Hasse-Witt. Soit $d$ la dimension de $G$. Le déterminant de la matrice de Hasse-Witt est l'invariant de Hasse $\mathrm{Ha}(G) \in\left(\Lambda^{d} \omega_{\mathrm{G}}\right)^{\otimes p-1}$.

Supposons que $R$ est un anneau $p$-adiquement complet. On note $\operatorname{Hdg}(G)$ et on appelle idéal de Hodge de $G$ l'image inverse dans $R$ de l'idéal $\mathrm{Ha}(G)\left(\Lambda^{d} \omega_{G}\right)^{\otimes(1-p)} \subset R / p$.

Lemme A.1. - L'idéal $\operatorname{Hdg}(G)$ est localement pour la topologie de Zariski engendrée par deux éléments. Supposons que $p \in \operatorname{Hdg}(G)^{2}$. Alors $\operatorname{Hdg}(G)$ est un idéal inversible.

Démonstration. - Quitte à remplacer Spec $R$ par un ouvert de Zariski, on peut supposer que l'idéal $\mathrm{Ha}(G)\left(\Lambda^{d} \omega_{G}\right)^{\otimes(1-p)} \subset R / p$ est principal. Notons Ha un relèvement dans $R$ d'un générateur. L'idéal de Hodge vaut alors ( $p$, Ha). Si $p \in \operatorname{Hdg}(G)^{2}$, alors $p=\tilde{\mathrm{Ha}} u+p^{2} v$ pour $u, v \in R$, et donc $p(1-p v)=\tilde{H} a u$. Comme $1-p v$ est inversible, $\operatorname{Hdg}(G)$ est engendré par $\tilde{\mathrm{Ha}}$.

\section{A.2. Construction du sous-groupe canonique}

Soit $A_{0}$ un anneau intègre et $\alpha \in A_{0}$ un élément non nul. On équipe $A_{0}$ de la topologie $\alpha$-adique et on suppose que $A_{0}$ est $\alpha$-adiquement complet. On suppose aussi qu'on a un morphisme $\mathbb{Z}_{p} \rightarrow A_{0}$ continu. Dans la théorie classique, on prend $A_{0}=\mathbb{Z}_{p}$ et $\alpha=p$, mais ici nous considérons une situation plus générale. On peut par exemple prendre $A_{0}=\mathbb{F}_{p}[[T]]$ et $\alpha=T$.

Proposition A.1. - Soit $R$ une $A_{0}$-algèbre $\alpha$-adiquement complète et sans $A_{0}$-torsion. Soit $G \rightarrow \operatorname{Spec} R$ un groupe fini et localement libre. Soit $C_{1} \subset G_{1}=G_{\left.\right|_{\operatorname{Spec}} R / p}$ un sousgroupe fini localement libre. Soit $L_{1}=G_{1} / C_{1}$. On suppose qu'il existe un élément $\lambda \in A_{0}$ tel que $p=\lambda^{2}$ u avec u topologiquement nilpotent et la multiplication par $\lambda$ est homotope à 0 sur le complexe de co-Lie $\ell_{L_{1} / R / p R}$. Alors il existe un sous-groupe fini et plat $C \subset G$ tel que $C_{\left.\right|_{\text {Spec } R / \frac{p}{\lambda} R}}=\left.C_{1}\right|_{\operatorname{Spec} R / \frac{p}{\lambda} R}$. 
Démonstration. - Introduisons les anneaux $B_{1}=R / p^{2} \lambda^{-1} R$ et $B_{2}=\{(x, y) \in$ $\left.R / p^{2} \lambda^{-2} R \times R / p R, x=y \bmod p \lambda^{-1} R\right\}$. On a des augmentations $B_{1} \rightarrow R / p R$ et $B_{2} \rightarrow$ $R / p R$ de noyaux respectifs $J_{1}$ égal à $p R / p^{2} \lambda^{-1} R$ et $J_{2}$ canoniquement isomorphe à $p \lambda^{-1} R / p^{2} \lambda^{-2}$. On identifie $J_{1}$ et $J_{2}$ à $R / p \lambda^{-1}$ en choisissant le générateur $p$ de $J_{1}$ et $p \lambda^{-1}$ de $J_{2}$. On a une application diagonale $B_{1} \rightarrow B_{2}$. Sa restriction $J_{1} \rightarrow J_{2}$ s'identifie à la multiplication par $\lambda$ dans $R / p \lambda^{-1}$. En appliquant le Thm. 3.2.1 (comme dans le coro. 3.2.2) de [26], on peut relever $\left.C_{1}\right|_{\operatorname{Spec} R / \frac{p}{\lambda} R}$ en un groupe localement libre $\left.C_{2} \hookrightarrow G\right|_{\operatorname{Spec} R / p^{2} \lambda-2 R}$. Supposons par récurrence qu'on a construit $C_{n} \hookrightarrow G_{\left.\right|_{\operatorname{Spec}} R / p^{n} \lambda^{-n} R}$ qui relève $\left.C_{1}\right|_{\operatorname{Spec} R / \frac{p}{\lambda} R}$.

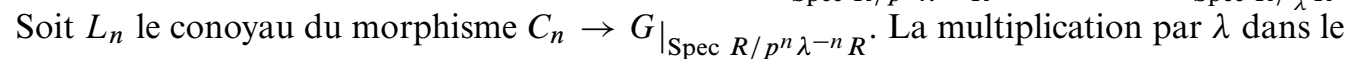
complexe de co-Lie $\ell_{L_{n} / R / p^{n} \lambda^{-n} R}$ est homotope à 0 modulo $\frac{p}{\lambda}$. On a donc, dans l'anneau des endomorphismes de $\ell_{L_{n} / R / p^{n}} \lambda^{-n}$ une homothopie $\lambda \sim \frac{p}{\lambda} v$ pour un endomorphisme $v$. Il en résulte que $\lambda(1-u v) \sim 0$, mais $1-u v$ est inversible car $u$ est topologiquement nilpotent. Il en résulte que la multiplication par $\lambda$ est homotope à 0 . Considérons alors les anneaux $B_{1}^{\prime}=R / p^{n+1} \lambda^{-n} R$ et $B_{2}^{\prime}=\left\{(x, y) \in R / p^{n+1} R \lambda^{-n+1} \times R / p^{n} \lambda^{-n} R, x=y \in\right.$ $\left.R / p^{n} \lambda^{-n-1} R\right\}$. On a des augmentations $B_{1}^{\prime} \rightarrow R / p^{n} \lambda^{-n}$ et $B_{2}^{\prime} \rightarrow R / p^{n} \lambda^{-n}$ de noyau $J_{1}^{\prime}$ et $J_{2}^{\prime}$ isomorphes à $R / p$. On a une application diagonale $B_{1}^{\prime} \rightarrow B_{2}^{\prime}$ qui induit la multiplication par $\lambda$ de $J_{1}$ vers $J_{2}$. En raisonnant comme au-dessus on construit un relèvement $\left.C_{n+1} \hookrightarrow G\right|_{\text {Spec } R / p^{n+1} \lambda^{-n-1} R}$ de $\left.C_{n}\right|_{\operatorname{Spec} R / p^{n} \lambda^{-n+1} R}$ et donc de $\left.C_{1}\right|_{\operatorname{Spec} R / \frac{p}{\lambda} R}$. Comme $R$ est $\frac{p}{\lambda}$-adiquement complet, on peut conclure.

Lemme A.2. - Soit $R$ une $A_{0}$-algèbre $\alpha$-adiquement complète et sans $A_{0}$-torsion. Soit $X \rightarrow \operatorname{Spec} R$ un schéma tel que $\Omega_{X / R}^{1}$ est annulé par la multiplication par $\lambda \in A_{0}$. Soit $a \in A_{0}$ tel que $a^{2} \in \lambda R$, et $s_{1}, s_{2}:$ Spec $R / a^{2} R \rightarrow X$ deux sections telles que $s_{1}=s_{2} \bmod a$. Alors $s_{1}=s_{2} \bmod a^{2} \lambda^{-1}$.

Démonstration. - Posons $s=s_{1} \bmod a$. L'ensemble des relèvements de $s$ en des sections modulo $a^{2}$ est un espace principal homogène sous $\operatorname{Hom}_{X}\left(\Omega_{X / R}^{1}, R / a\right.$ ) (où $R / a=a / a^{2} R$ est vu comme un $\mathscr{O}_{\mathrm{X}}$-module au moyen de la section $s$ ). De même, l'ensemble des relèvements modulo $a^{2} \lambda^{-1}$ de $s$ est un espace principal homogène sous $\operatorname{Hom}_{X}\left(\Omega_{X / R}^{1}, R / a \lambda^{-1}\right)$. L'application $\operatorname{Hom}_{X}\left(\Omega_{X / R}^{1}, R / a\right) \rightarrow \operatorname{Hom}_{X}\left(\Omega_{X / R}^{1}, R / a \lambda^{-1}\right)$ correspond à la restriction des sections de $R / a^{2}$ à $R / a^{2} \lambda^{-1}$, et elle est nulle car $\lambda$ annule $\Omega_{X / R}^{1}$. Cela signifie que tous les relèvements à $R / a^{2}$ sont égaux dans $R / a^{2} \lambda^{-1}$.

Corollaire A.1. - Soit $R$ une $A_{0}$-algèbre $\alpha$-adiquement complète et sans $A_{0}$-torsion. Soit $G$ un groupe de Barsotti-Tate tronqué d'échelon $n$ et dimension d sur $R$. Soit $\lambda \in A_{0}$ tel que $\lambda^{2} u=p$ avec u topologiquement nilpotent. Soit $m \leq n$. Supposons que $\lambda \bmod p \in$ $\mathrm{Ha}(G)^{\frac{p^{m}-1}{p-1}}\left(\Lambda^{d} \omega_{\mathrm{G}}\right)^{\otimes\left(1-p^{m}\right)}$. Alors, $G$ possède un sous-groupe fini localement libre $H_{m} q u i$ vérifie les propriétés suivantes :

1. $H_{m}$ relève $\operatorname{Ker} F^{m}$ modulo $\frac{p}{\lambda}$,

2. pour toute $R$-algèbre $R^{\prime}, \alpha$-adiquement complète et sans $A_{0}$-torsion,

$$
H_{m}\left(R^{\prime}\right)=\left\{s \in G\left[p^{m}\right]\left(R^{\prime}\right), s \bmod p \lambda^{-1} \in \operatorname{Ker} F^{m}\right\},
$$

3. soit $L_{m}=G\left[p^{m}\right] / H_{m}$. Alors $\omega_{L_{m}}$ est annulé par $\operatorname{Hdg}(G)^{\frac{p^{m}-1}{p-1}}$ et on a des isomorphismes $\operatorname{det} \omega_{L_{m}} \simeq \operatorname{det} \omega_{\mathrm{G}} / \operatorname{Hdg}(G)^{\frac{p^{m}-1}{p-1}}$. 
Remarque A.1. - La seconde propriété caractérise $H_{m}$.

Définition A.1. - Le groupe $H_{m} d u$ corollaire A.1 est appelé sous-groupe canonique d'échelon $m$ de $G$.

Démonstration. - Quitte à faire une localisation de Zariski, on peut supposer que le faisceau $\omega_{\mathrm{G}}$ est trivial. Soit $G_{1}=G_{\left.\right|_{R / p}}$. Soit $\operatorname{Ker} V^{m}$ le conoyau de l'inclusion $\operatorname{Ker} F^{m} \rightarrow G_{1}\left[p^{m}\right]$. Le complexe de co-Lie de $\operatorname{Ker} V^{m}$ vaut :

$$
\left[\omega_{G_{1}} \stackrel{\operatorname{HW}(G)^{\frac{p^{m}-1}{p-1}}}{\longrightarrow} \omega_{G_{1}^{\left(p^{m}\right)}}\right] .
$$

Comme $\mathrm{Ha}(G)^{\frac{p^{m}-1}{p-1}}$ divise $\lambda$, la multiplication par $\lambda$ est homotope à 0 . On peut donc appliquer la proposition A.1. Ceci démontre l'existence de $H_{m}$. Soit $L_{m}$ le conoyau du morphisme $H_{m} \rightarrow G\left[p^{m}\right]$. Comme $L_{m}$ se réduit sur $\operatorname{Ker} V^{m}$ modulo $p \lambda^{-1}$, on obtient que $\omega_{L_{m}}$ est annulé par $\operatorname{Hdg}(G)^{\frac{p^{m}-1}{p-1}}$ modulo $p \lambda^{-1}$. Il en résulte que $\operatorname{Hdg}(G)^{\frac{p^{m}-1}{p-1}} \omega_{L_{m}} \subset$ $\operatorname{Hdg}(G)^{\frac{p^{m}-1}{p-1}} u \omega_{L_{m}}$ et le lemme de Nakayama entraîne que $\omega_{L_{m}}$ est annulé $\operatorname{par} \operatorname{Hdg}(G)^{\frac{p^{m_{-1}}}{p-1}}$ et donc aussi par $\lambda$.

On a une surjection det $\omega_{\mathrm{G}} \rightarrow \operatorname{det} \omega_{L_{m}}$. qui induit un isomorphisme

$$
\operatorname{det} \omega_{\mathrm{G}} /\left(\operatorname{Hdg}(G)^{\frac{p^{m_{-}}}{p-1}}, p \lambda^{-1}\right) \rightarrow \operatorname{det} \omega_{L_{m}} .
$$

Comme $p \lambda^{-1} \in \operatorname{Hdg}(G)^{\frac{p^{m}-1}{p-1}}$ on obtient l'isomorphisme det $\omega_{L_{m}} \simeq \operatorname{det} \omega_{\mathrm{G}} / \operatorname{Hdg}(G)^{\frac{p^{m}-1}{p-1}}$.

Démontrons la formule $H_{m}\left(R^{\prime}\right)=\left\{s \in G\left[p^{m}\right]\left(R^{\prime}\right), s \bmod p \lambda^{-1} \in \operatorname{Ker} F^{m}\right\}$. Il résulte du lemme A.2 que si $s \in L_{m}\left(R^{\prime}\right)$ et la section unité modulo $p \lambda^{-1}$, elle est la section unité modulo $p^{2} \lambda^{-3}=\frac{p}{\lambda} u$. En répétant l'argument, on en déduit que c'est la section unité modulo $\frac{p}{\lambda} u^{r}$ pour tout $r$ et donc la section unité.

Remarque A.2. - Si $R$ est une $\mathbb{F}_{p}$-algèbre la théorie est bien sûre triviale, $G$ possède toujours un sous-groupe canonique d'ordre $n$ : le noyau de $F^{n}$. On le retrouve en appliquant la proposition avec $\lambda=0$ et $u=0$.

Corollaire A.2. - Soit $R$ une $A_{0}$-algèbre $\alpha$-adiquement complète et sans $A_{0}$-torsion. Soit $k \in \mathbb{Z}_{\geq 0}$. Soit $G$ un groupe de Barsotti-Tate sur $R$, de dimension $d$ et hauteur $h$. Soit $m \in \mathbb{Z}_{\geq 0}$. Supposons que $p \in \operatorname{Hdg}(G)^{p^{m+1}}$.

1. G possède un sous-groupe canonique $H_{\mathrm{n}}$ d'échelon n pour tout $n \leq$ m, et on a $H_{n} \subset H_{n^{\prime}}$ si $n \leq n^{\prime}$.

2. $H_{n}$ est localement libre de rang $p^{\text {nd }}$ et il relève le groupe $\operatorname{Ker} F^{n}$ modulo $p \operatorname{Hdg}(G)^{-\frac{p^{n}-1}{p-1}}$.

3. Soit $G^{\prime}=G / H_{n}$. Alors $\operatorname{Hdg}\left(G^{\prime}\right)=\operatorname{Hdg}(G)^{p^{n}}, G^{\prime}$ possède un sous-groupe canonique $H_{m-n}^{\prime}$, d'échelon $m-n$, et on a une suite exacte :

$$
0 \rightarrow H_{n} \rightarrow H_{m} \rightarrow H_{m-n}^{\prime} \rightarrow 0 .
$$

4. Le faisceau conormal $\omega_{G\left[p^{n}\right] / H_{n}}$ est annulé par $\operatorname{Hdg}(G)^{\frac{p^{n}-1}{p-1}}$ et $\operatorname{det} \omega_{G\left[p^{n}\right] / H_{n}} \simeq$ $\operatorname{det} \omega_{G\left[p^{n}\right]} / \operatorname{Hdg}(G)^{\frac{p^{n}-1}{p-1}}$.

5. Soi $G^{D}$ le dual de $G$. Alors $\operatorname{Hdg}(G)=\operatorname{Hdg}\left(G^{D}\right)$. Pour tout $n \leq m$, l'accouplement $G\left[p^{n}\right] \times G\left[p^{n}\right]^{D} \rightarrow \mu_{p^{n}}$ induit une identification $H_{n}(G) \simeq H_{n}\left(G^{D}\right)^{\perp}$. 
6. Supposons de plus que $\alpha \in \operatorname{Hdg}(G)$. Alors $G\left[p^{n}\right] / H_{n}$ est étale sur $\operatorname{Spec} R[1 / \alpha]$, localement isomorphe à $\left(\mathbb{Z} / p^{m} \mathbb{Z}\right)^{h-d}$.

Remarque A.3. - Le caractère étale de $G\left[p^{m}\right] / H_{m}$ n'est pas complètement trivial puisque $p$ n'est pas forcément inversible dans $R\left[\frac{1}{\alpha}\right]$. Il suffit de penser à la situation où $A_{0}=\mathbb{F}_{p}[[T]], \alpha=T$.

Démonstration. - Quitte à faire une localisation Zariski, on peut supposer que $\operatorname{Hdg}(G)$ est principal engendré par Ha. On pose $\lambda=\tilde{\mathrm{Ha}} \tilde{\frac{p}{p-1}}^{\frac{p^{m}-1}{2}}, u=p \tilde{\mathrm{Ha}}^{-2 \frac{p^{m}-1}{p-1}}$. On vérifie que $u$ est topologiquement nilpotent car $p^{m+1}>2 \frac{p^{m}-1}{p-1}$. En effet, par hypothèse il existe $v \in R$ tel que $p=v \tilde{\mathrm{Ha}}^{p^{m+1}}$. Ainsi

$$
u=v \tilde{\mathrm{Ha}}{ }^{p^{m+1}-2 \frac{p^{m}-1}{p-1}}=v \tilde{\mathrm{Ha}} \tilde{\mathrm{Ha}}^{p^{m+1}-2 \frac{p^{m}-1}{p-1}-1}
$$

et il suffit de voir que $v \tilde{\mathrm{H}}$ e est topologiquement nilpotent. Comme $(v \tilde{\mathrm{Ha}})^{p^{m+1}}=p v^{p^{m+1}-1}$, c'est clair. L'existence de $H_{n}$ pour $n \leq m$, l'inclusion $H_{n} \subset H_{m}$ et la propriété de relèvement du noyau de Frobenius résultent du corollaire A.1.

Si $G^{\prime}=G / H_{n}$, on a $G^{\prime}=G^{\left(p^{n}\right)}$ modulo $p \tilde{\mathrm{Ha}}^{-\frac{p^{n}-1}{p-1}}$ et on a donc l'égalité des idéaux :

$$
\left(\operatorname{Hdg}\left(G^{\prime}\right), p \tilde{\mathrm{Ha}}^{-\frac{p^{n}-1}{p-1}}\right)=\left(\operatorname{Hdg}(G)^{p^{n}}, p \tilde{\mathrm{Ha}}^{-\frac{p^{n}-1}{p-1}}\right) .
$$

Vérifions alors que $\operatorname{Hdg}\left(G^{\prime}\right)$ est engendrée par $\tilde{\mathrm{Ha}}^{p^{n}}$. Soit $x \in \operatorname{Hdg}\left(G^{\prime}\right)$ et $u \in R$ tels que $x=\tilde{\mathrm{Ha}}{ }^{p^{n}}+u p \tilde{\mathrm{Ha}}{ }^{-\frac{p^{n}-1}{p-1}}$. Alors $x=\tilde{\mathrm{Ha}}^{p^{n}}\left(1+u p \tilde{\mathrm{Ha}}{ }^{-\frac{p^{n+1}-1}{p-1}}\right)$. Or $p \tilde{\mathrm{Ha}}{ }^{-\frac{p^{n+1}-1}{p-1}}$ est topologiquement nilpotent, donc $\tilde{\mathrm{Ha}}^{p^{n}} \in \operatorname{Hdg}\left(G^{\prime}\right)$ et c'est clairement un générateur. Il en résulte que $\operatorname{Hdg}\left(G^{\prime}\right)$ possède un sous-groupe canonique d'échelon $m-n$. On a une application évidente $H_{m} \rightarrow H_{m-n}^{\prime}$ d'après la propriété 2 du corollaire $A .1$. En comparant les ordres de $H_{n}, H_{m}$ et $H_{n-m}^{\prime}$, on obtient la suite exacte annoncée. Soit $G^{D}$ le dual de $G$. On a $\operatorname{Hdg}(G)=\operatorname{Hdg}\left(G^{D}\right)$ d'après [15]. Considérons le groupe $H_{n}(G)^{\perp} \subset G^{D}\left[p^{n}\right]$. Le point 2. du corollaire A.1 montre que $H_{n}(G)^{\perp} \subset H_{n}\left(G^{D}\right)$. Comme ces deux groupes ont même rang, ils sont égaux. Vérifions le dernier point. Posons $L_{m}=G\left[p^{m}\right] / H_{m}$. Comme $\omega_{L_{m}}$ est de $\alpha$-torsion, il en résulte que $L_{m}$ est étale sur Spec $R\left[\frac{1}{\alpha}\right]$. La dernière propriété est vérifiée sur une partie ouverte et fermée de Spec $R\left[\frac{1}{\alpha}\right]$. Soit $x$ un idéal de Spec $R\left[\frac{1}{\alpha}\right]$. Nous allons construire un morphisme $R / x \rightarrow O_{K}$ où $O_{K}$ est un anneau de valuation complet pour une valuation de rang 1 , tel que l'image de $\alpha$ est une pseudo-uniformisante. Soit $x^{\prime}$ un idéal maximal de Spec $R$ qui est une spécialisation de $x$. Remarquons que $\alpha \in x$. Soit $A$ un sous anneau de valuation de $\operatorname{Frac}\left(R_{x^{\prime}} / x\right)$ qui domine $R_{x^{\prime}} / x$. Cela nous fournit une valuation $v$ sur $R\left[\frac{1}{\alpha}\right]$. D'après [18], prop. 2.6 (appliquée à $I=R^{00}$ ) et Thm. 3.1, il existe une spécialisation $v^{\prime} \in \operatorname{Spa}\left(R\left[\frac{1}{\alpha}\right], \tilde{R}\right)$ de $v$, où $\tilde{R}$ est la normalisations de $R$ dans $R\left[\frac{1}{\alpha}\right]$. Le support dans Spec $R\left[\frac{1}{\alpha}\right]$ de $v^{\prime}$ est une spécialisation de $x$. D'autre part, comme $R\left[\frac{1}{\alpha}\right]$ est une algèbre de Tate, $v^{\prime}$ admet une générisation de rang 1. On a donc un morphisme $R \rightarrow O_{K}$ où $K$ est un corps valué complet pour une valuation de rang 1 et $O_{K}$ est son anneau d'entiers. Si $K$ est un corps de caractéristique $p,\left.L_{m}\right|_{K}=\operatorname{Ker} V^{m}$ et $\left.L_{m}\right|_{K}$ est bien localement isomorphe à $\left(\mathbb{Z} / p^{m} \mathbb{Z}\right)^{h-d}$. Sinon, $K$ est un corps local d'inégale caractéristique, la théorie classique (voir [15] par exemple), entraîne que $\left.L_{m}\right|_{K}$ est localement isomorphe à $\left(\mathbb{Z} / p^{m} \mathbb{Z}\right)^{h-d}$. Il en 
résulte que $L_{m}$ est localement libre comme $\mathbb{Z} / p^{m} \mathbb{Z}$-module en tout point fermé de Spec $R\left[\frac{1}{\alpha}\right]$ et donc sur Spec $R\left[\frac{1}{\alpha}\right]$ tout entier.

\section{A.3. Sous-groupes canoniques et application de Hodge-Tate}

Dans [15], le sous-groupe canonique est construit comme noyau de certaines applications de Hodge-Tate. Faisons le lien avec cette approche.

Rappelons d'abord un résultat classique. Soit $R$ une $\mathbb{F}_{p}$-algèbre et $G$ un groupe de Barsotti-Tate d'échelon 1, de hauteur $h$ et dimension $d$. On a alors une suite exacte de schémas en groupes (voir par exemple [15], sect. 2.1.2.) :

$$
0 \rightarrow \operatorname{Ker} F \rightarrow G \stackrel{\mathrm{HT}}{\rightarrow} \underline{\omega}_{G^{D}} \stackrel{F-\mathrm{HW}\left(G^{D}\right)}{\rightarrow} \underline{\omega}_{G^{\mathrm{D}}}^{(p)} \rightarrow 0 .
$$

Ici, $\underline{\omega}_{G}{ }^{D}$ et $\underline{\omega}_{G D}^{(p)}$ sont les schémas en groupes vectoriels associés aux faisceaux localement libres $\omega_{G^{D}}$ et $\omega_{G^{D}}^{(p)}$. Ils sont localement pour la topologie de Zariski isomorphes à $\mathbb{G}_{a}^{h-d}$. De plus, HT désigne l'application de Hodge-Tate

On se place à présent sous les hypothèses du corollaire $\mathrm{A} .1: A_{0}$ est une $\mathbb{Z}_{p}$-algèbre intègre, $\alpha \in A_{0} \backslash\{0\}, A_{0}$ est $\alpha$-adiquement complète et le morphisme structural $\mathbb{Z}_{p} \rightarrow A_{0}$ est continu. On se donne $R$ une $A_{0}$-algèbre $\alpha$-adiquement complète et sans $A_{0}$-torsion et $G$ un groupe de Barsotti-Tate sur $R$ d'échelon 1, de hauteur $h$ et dimension $d$. Soit $\lambda \in A_{0}$ tel que $\lambda^{2} u=p$ avec $u$ topologiquement nilpotent. Supposons que $\lambda \bmod p \in \operatorname{Ha}(G)\left(\Lambda^{d} \omega_{\mathrm{G}}\right)^{\otimes(1-p)}$. Soit $H_{1}$ le sous-groupe canonique d'échelon 1.

Proposition A.2. - Pour toute $R$-algèbre $R^{\prime}, \alpha$-adiquement complète et sans $A_{0}$-torsion, on a une suite exacte :

$$
0 \rightarrow H_{1}\left(R^{\prime}\right) \rightarrow G[p]\left(R^{\prime}\right) \stackrel{\mathrm{HT}}{\rightarrow} \omega_{G^{D}} \otimes_{R} R^{\prime} / p \lambda^{-1} R^{\prime} .
$$

Démonstration. - On a un diagramme commutatif

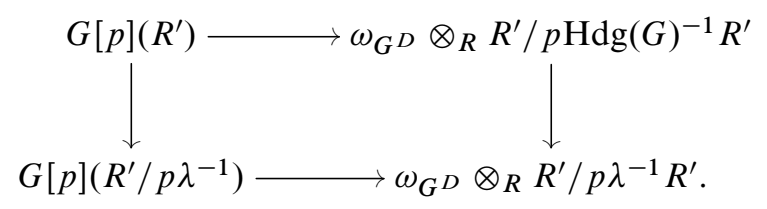

Soit $x \in G[p]\left(R^{\prime}\right)$. Alors $x \in H_{1}\left(R^{\prime}\right)$ si et seulement si $x \bmod p \lambda^{-1} \in \operatorname{Ker} F$ d'après le corollaire A.1. On vient de voir que $x \bmod p \lambda^{-1} \in \operatorname{Ker} F$ si et seulement si $\mathrm{HT}(x) \bmod p \lambda^{-1}=0$.

On suppose à présent que $\alpha=p$, que $R$ est normal et que $p \in \operatorname{Hdg}(G)^{p^{2}}$. On suppose de plus que $G / H_{1}(R)=(\mathbb{Z} / p \mathbb{Z})^{h-d}$. On considère la puissance extérieure maximale de l'application de Hodge-Tate :

$$
\operatorname{det} G / H_{1}(R) \stackrel{\operatorname{det} H T}{\rightarrow} \operatorname{det} \omega_{G^{D}} \otimes_{R} R / p \operatorname{Hdg}(G)^{-1} R=\operatorname{det} \omega_{\left(G / H_{1}\right)^{D}} \otimes_{R} R / p \operatorname{Hdg}(G)^{-1} R .
$$

Soit $P$ une base du $\mathbb{F}_{p}$-espace vectoriel $\operatorname{det} G / H_{1}(R)$. Soit $I$ l'idéal de $R / p \operatorname{Hdg}(G)^{-1}$ défini par :

$$
\operatorname{det} \operatorname{HT}(P) \text {. det } \omega_{G^{D}} \otimes_{R} R / p \operatorname{Hdg}(G)^{-1} R=I\left(\operatorname{det} \omega_{G^{D}} \otimes_{R} R / p \operatorname{Hdg}(G)^{-1} R\right) .
$$

On note $\operatorname{HdgT}(G)$ et on appelle idéal de Hodge-Tate de $G$ l'image inverse de $I$ dans $R$. 
Proposition A.3. - L'idéal $\operatorname{HdgT}(G)$ est inversible et on a l'égalité des idéaux $\operatorname{HdgT}(G)^{p-1}=\operatorname{Hdg}(G)$.

Démonstration. - Comme $R$ est normal, on se ramène facilement au cas où $R$ est un anneau de valuation complet pour une valuation de rang 1 et $p$ est une pseudouniformisante. C'est alors une conséquence de [15], prop. 7.

\section{Appendice B}

\section{Théorie spectrale}

Nous étendons la théorie spectrale de Coleman afin de pouvoir l'appliquer au-dessus de l'espace des poids $\mathscr{W}$. Le point nouveau essentiel est l'existence de factorisations locales des séries de Fredholm sur un espace adique général. Seul le cas des espaces rigides avait été étudié auparavant. Fixons $\left(A, A^{+}\right)$une $\mathbb{Z}_{p}$-algèbre de Tate. Notons $A_{0} \subset A^{+}$un anneau de définition et fixons $\alpha \in A_{0}$ tel que $A=A_{0}[1 / \alpha]$ et $(\alpha)$ est un idéal de définition dans $A_{0}$. Nous supposons de plus que $A_{0}$ est noethérien, et donc $\left(A, A^{+}\right)$est faisceautique d'après [19], Thm. 2.5.

\section{B.1. Série de Fredholm et factorisation}

Dans ce numéro nous introduisons les séries de Fredholm, leurs variétés spectrales et établissons leurs factorisations locales.

Définition B.1. - Une série formelle $F=\sum_{n \geq 0} a_{n} T^{n} \in A[[T]]$ est appelée série de Fredholm si :

1. $a_{0}=1$,

2. pour tout $r \in \mathbb{Z}, a_{n} \alpha^{r n} \rightarrow 0$ quand $n \rightarrow \infty$.

Soit $\mathbb{A}^{1}$ la droite affine sur $\operatorname{Spa}\left(A, A^{+}\right)$. C' est la réunion croissante des boules affinoïdes $\left\{\mathbb{B}_{n}=\operatorname{Spa}\left(A\left\langle\alpha^{\mathrm{n}} T\right\rangle, A^{+}\left\langle\alpha^{\mathrm{n}} T\right\rangle\right)\right\}_{n \in \mathbb{N}}$ d'équation $\left|\alpha^{n} T\right| \leq 1$. Plus généralement, pour tout rationnel positif $\lambda=\frac{r}{s}$, on note $\mathbb{B}_{\lambda}$ l'ouvert affinoïde de $\mathbb{A}^{1}$ d'équation $\left|T^{s}\right| \leq\left|\alpha^{-r}\right|$. Soit $F$ une série de Fredholm. Pour tout $n \in \mathbb{N}$, on peut définir le fermé $V(F)_{n} \subset \mathbb{B}_{n}$ où $V(F)_{n}=\operatorname{Spa}\left(C_{n}, C_{n}^{+}\right)$avec $C_{n}=A\left\langle\alpha^{n} T\right\rangle /(F)$ et $C_{n}^{+}$est la normalisation de l'image de $A^{+}\left\langle\alpha^{n} T\right\rangle$ dans $C_{n}$.

Définition B.2. - La variété spectrale $V(F)$ est le fermé de $\mathbb{A}^{1}$ qui vaut la réunion des $V(F)_{n}$.

On a un morphisme structural $w: V(F) \rightarrow \operatorname{Spa}\left(A, A^{+}\right)$. La construction de la variété spectrale commute au changement de base.

Rappelons quelques définitions tirées de [20], sect. 1.3, 1.4. et 1.5. Un morphisme $\left(C, C^{+}\right) \rightarrow\left(B, B^{+}\right)$d'algèbres affinoïdes est fini si $B$ est fini sur $C$ et $B^{+}$est la clôture intégrale de $C^{+}$dans $B$. Il est fini et plat si $B$ est de plus plat sur $C$. Un morphisme $f: X \rightarrow Y$ d'espaces adiques est fini et plat si tout point $y \in Y$ possède un voisinage affine $U=\mathrm{Spa}\left(C, C^{+}\right)$tel que $f^{-1}(U)=\mathrm{Spa}\left(B, B^{+}\right)$est affine et $\left(C, C^{+}\right) \rightarrow\left(B, B^{+}\right)$est un morphisme fini et plat. Un morphisme localement de type fini d'espaces adiques analytiques $f: X \rightarrow Y$ est localement quasi-fini si ses fibres sont discrètes. Enfin, un morphisme 
localement de type fini quasi-séparé d'espaces adiques analytiques est partiellement propre s'il vérifie le critère valuatif de propreté de [20], coro. 1.3.9 (voir aussi lem. 1.3.10).

Lemme B.1. - Supposons que A est un corps. Il existe alors une suite strictement croissante tendant vers l'infini $\left(\lambda_{i}\right) \in \mathbb{Q}_{>0}^{\mathbb{N}}$ telle que $V(F) \cap \mathbb{B}_{\lambda_{i}}$ soit fini et plat sur $\operatorname{Spa}\left(A, A^{+}\right)$.

Démonstration. - Soit $A^{0}$ l'anneau des éléments à puissance bornée dans $A$ et $A^{00}$ l'idéal des éléments topologiquement nilpotents. On a $A^{00} \subset A^{+} \subset A^{0}$. Soit |.| la norme de rang 1 sur $A$ correspondant à $A^{0}$ et $v$ la valuation associée. On normalise $v$ par $v(\alpha)=1$. Soit $\left\{s_{i}\right\}_{i \in \mathbb{N}}$ les pentes du polygone de Newton de $F$, sans multiplicité, rangées par ordre strictement croissant. Soit $n \in \mathbb{N}$. Soit $\lambda_{n}=\frac{l}{m}$ un rationnel tel que $s_{n}<\lambda_{n}<s_{n+1}$. Rappelons que $\mathbb{B}_{\lambda_{n}} \subset \mathbb{A}^{1}$ est l'ouvert rationnel d'équation $\left|\alpha^{l} T^{m}\right| \leq 1$. Nous allons montrer que $\mathbb{B}_{\lambda_{n}} \cap V(F) \rightarrow S$ est fini. Quitte à faire une extension finie de $A$, ce qui est loisible d'après [20], lem. 1.4.9, on peut supposer qu'il existe $\beta \in A$ tel que $v(\beta)=\lambda_{n}$. On a alors $\mathbb{B}_{\lambda}=\operatorname{Spa}\left(A\langle\beta T\rangle, A^{+}\langle\beta T\rangle\right)$. Soit la factorisation de $F$ en $F=P U$, où $P$ est le polynôme de polygone de Newton de pentes strictement inférieures à $\lambda_{n}$ et $U$ est la série de Fredholm de polygone de Newton de pentes strictement supérieures à $\lambda_{n}$. Dans une clôture algébrique $\bar{A}$ de $A$, on a $P(T)=\prod\left(1-a_{i} T\right)$ avec $v\left(a_{i}\right)<\lambda_{n}$, donc $P(T)=\prod_{i} \beta^{-1} a_{i} \prod_{i}\left(a_{i}^{-1} \beta-\beta T\right)$. Posons $Q(T)=\prod\left(a_{i}^{-1} \beta-\beta T\right) \in A^{00}\langle\beta T\rangle$. Introduisons aussi la variable $S=\beta T$. On a alors $A\langle\beta T\rangle /(F(T))=A\langle\beta T\rangle /(Q(T))=A\langle S\rangle /(Q(S))$ et $(A\langle\beta T\rangle / F(T))^{+}=$ $A^{+}\langle S\rangle /(Q(S))$ et ces algèbres sont finies sur $A$ et $A^{+}$respectivement.

ThÉORÈme B.1. - Le morphisme $V(F) \rightarrow \operatorname{Spa}\left(A, A^{+}\right)$est localement quasi-fini, plat et partiellement propre. Pour tout $x \in V(F)$ et $w(x) \in \mathrm{Spa}\left(A, A^{+}\right)$, il existe un voisinage $U$ de $x$ dans $V(F)$ tel que $\overline{\{x\}} \subset U$ et un voisinage $V$ de $w(x)$ dans $\operatorname{Spa}\left(A, A^{+}\right)$tel que $w(U) \subset V$ et le morphisme $\left.w\right|_{\mathrm{U}}: U \rightarrow V$ est fini et plat.

Démonstration. - Le morphisme $V(F) \rightarrow \operatorname{Spa}\left(A, A^{+}\right)$est localement de type fini et quasi-séparé par construction. D'autre part, il est localement quasi-fini et vérifie le critère valuatif de propreté ([20], coro. 1.3.9) d'après le lemme B.1. D'après la proposition 1.5.6 de [20], pour tout $x \in V(F)$ et $w(x) \in \operatorname{Spa}\left(A, A^{+}\right)$, il existe un voisinage $U$ de $x$ dans $V(F)$ tel que $\overline{\{x\}} \subset U$ et un voisinage $V$ de $w(x)$ dans $\operatorname{Spa}\left(A, A^{+}\right)$tel que le morphisme $\left.w\right|_{\mathrm{U}}: U \rightarrow V$ est fini. Par ailleurs, pour tout $n$, on voit comme dans [7], Part I, lem. 4.1, que le morphisme $V(F)_{n} \rightarrow \operatorname{Spa}\left(A, A^{+}\right)$est plat. D'autre part $\left.U \hookrightarrow V(F)_{n}\right|_{\mathrm{V}}$ pour $n$ suffisamment grand et comme $U$ est propre sur $V, U$ est ouvert et fermé dans $V(F)_{n}$, donc c'est une union de composantes connexes de $V(F)_{n}$. Par conséquent, le morphisme $U \rightarrow V$ est plat.

Corollaire B.1. - Soit $x \in V(F)$, U et $V$ comme dans le théorème. On suppose (il suffit de rapetisser $V)$ que $U=\operatorname{Spa}\left(C, C^{+}\right), V=\operatorname{Spa}\left(B, B^{+}\right)$, et que $U$ est de rang constant $d$ sur $V$. Alors la série de Fredholm $F$ possède une factorisation à coefficients dans $B: F=Q S$ où $Q=1+b_{1} T+\cdots+b_{d} T^{d} \in B[T], b_{d} \in B^{\times}$, S est une série de Fredholm à coefficients dans $B$ première à $Q$ et $C=B[T] / Q(T)$.

Démonstration. - Comme $U$ est un fermé de $V(F)_{n}$ pour $n$ assez grand, on possède une surjection $B<\alpha^{n} T>\rightarrow C$. Soit $L \in B[T]$ le polynôme caractéristique de l'image $T$ dans $C$. Le composé $B[T] /(L) \rightarrow B<\alpha^{n} T>/(L) \rightarrow C$ est un morphisme de $B$-modules de type fini. La topologie de $B$ induit sur ces modules une topologie canonique ([19], p. 524). La 
topologie sur $C$ est aussi la topologie quotient induite par la surjection $B<\alpha^{n} T>\rightarrow C$ d'après la proposition B.1. Le morphisme $B[T] /(L) \rightarrow C$ est donc d'image dense et comme il est strict ([19], lem. 2.3), il est surjectif. En comparant les rangs, on déduit que ce morphisme est un isomorphisme. On a une factorisation $F=L M$ dans $B<\alpha^{n} T>$ pour tout $n$ assez grand. Il en résulte que $L(0) \in B^{\times}$. Posons alors $Q(T)=L(0)^{-1} L(T)$. On a $F=Q S$ avec $S=L(0) M$. De plus $Q$ et $S$ sont premiers entre eux car $U$ est ouvert et fermé dans $V(F)_{n}$ pour tout $n$ assez grand.

\section{B.2. Endomorphismes complètement continus d'un module de Banach}

B.2.1. Algèbres de Tate et algèbres de Banach. - On rappelle que $A$ est une algèbre de Tate, $A_{0} \subset A$ un anneau de définition, et que $\alpha \in A_{0}$ est une unité topologiquement nilpotente. Nous supposons aussi que $A_{0}$ est noethérien.

On peut munir $A$ d'une norme $||:. A \rightarrow \mathbb{R}_{\geq 0}$ en posant $|a|=\inf _{n, \alpha^{n} a \in A_{0}} p^{n}$. On vérifie immédiatement les propriétés suivantes :

1. pour tout $a, b \in A,|a+b| \leq \sup \{|a|,|b|\}$,

2. pour tout $a, b \in A,|a b| \leq|a||b|$,

3. pour tout $n \in \mathbb{Z}$ et $a \in A,\left|\alpha^{n} a\right|=p^{-n}|a|$,

4. pour tout $n \in \mathbb{Z},|a| \leq p^{-n} \Leftrightarrow a \in \alpha^{n} A_{0}$.

En particulier, la topologie induite par la norme sur $A$ est sa topologie d'origine.

B.2.2. Modules de Banach. - On dit qu'un $A$-module topologique $M$ est un module de Banach s'il possède un sous- $A_{0}$ module $M_{0}$ tel que $M=M_{0}[1 / \alpha]$ et $M_{0}$ est ( $\alpha$ )-adiquement complet et séparé. Si $M$ est un $A$-module de Banach, alors on peut le munir d'une norme |.| $: M \rightarrow \mathbb{R}_{\geq 0}$ en posant $:|m|=\inf _{n, \alpha^{n} M \in M_{0}} p^{n}$. Cette norme nous redonne la topologie de $M$. On note $\operatorname{Ban}(A)$ la catégorie qui a pour objets les $A$-modules de Banach et pour morphismes les applications $A$-linéaires continues.

Le théorème de l'application ouverte est valable dans ce contexte.

Proposition B.1. - Soit $f: M \rightarrow N$ une application A-linéaire continue surjective entre deux A-modules de Banach. Alors $f$ est ouverte.

Démonstration. - Soit $M_{0}$ (resp. $N_{0}$ ) un sous $A_{0}$ module de $M$ (resp. $N$ ) tel que $M_{0}$ (resp. $N_{0}$ ) est $\alpha$-adiquement complet et $M=M_{0}[1 / \alpha]$ (resp. $N=N_{0}[1 / \alpha]$ ). D'après le théorème de Baire, il existe $n, \alpha^{n} N_{0} \subset \overline{f\left(M_{0}\right)}$. Soit $v \in \alpha^{n} N_{0}$. On peut donc trouver $x_{1} \in M_{0}$ tel que $f\left(x_{1}\right)-v \in \alpha^{2 n} N_{0}$. Posons $v_{2}=\alpha^{-n}\left(f\left(x_{1}\right)-u\right) \in \alpha^{n} N_{0}$. On peut alors trouver $x_{2} \in M_{0}$ tel que $f\left(x_{2}\right)-v_{2} \in \alpha^{2 n} N_{0}$. En répétant, on construit ainsi une suite $\left(x_{i}\right)_{i \geq 1}$ telle que $f\left(\sum_{i=1}^{k} \alpha^{(i-1) n} x_{i}\right)-v \in \alpha^{(k+1) n} N_{0}$. Posons donc $x=\sum_{i=1}^{\infty} \alpha^{(i-1) n} x_{i}$. Alors $f(x)=v$ et donc $\alpha^{n} N_{0} \subset f\left(M_{0}\right)$.

Rappelons également que d'après [19], lem. 2.3, la catégorie des $A$-modules de type fini est une sous-catégorie pleine de la catégorie $\operatorname{Ban}(A)$.

Proposition B.2. - Tout A-module de type fini $M$ possède une unique topologie qui en fait un A-module de Banach. Tout morphisme $f: M \rightarrow N$ entre deux A-modules de type fini est continu, $f(M)$ est fermé dans $N$, et le morphisme induit $M \rightarrow f(M)$ est ouvert. 
Exemple 2. - Soit $I$ un ensemble. On note $c_{A}(I)$ le $A$-module des fonctions $f: I \rightarrow A$ qui vérifient $\lim _{i \rightarrow \infty} f(i)=0$. On l'équipe de la norme supremum $|f|=\sup _{i \in I}|f(i)|$. Alors $c_{A}(I)$ est un module de Banach. On notera $e_{i} \in c_{A}(I)$ la fonction caractéristique de $i \in I$. Les éléments $\left(e_{i}\right)_{i \in I}$ forment une base topologique de $c_{A}(I)$.

On dit qu'un $A$-module de Banach $M$ est orthonormalisable s'il existe un ensemble $I$ et un homéomorphisme : $c_{A}(I) \rightarrow M$. On vérifie facilement que tout module de Banach est un quotient d'un module orthonormalisable. On dit qu'un $A$-module de Banach $M$ est projectif si c'est un facteur direct d'un $A$-module orthonormalisable. Un $A$-module de Banach est projectif si et seulement s'il vérifie la propriété universelle suivante : Tout diagramme de morphismes dans $\operatorname{Ban}(A)$ :

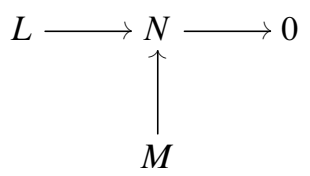

peut être complété en un diagramme commutatif

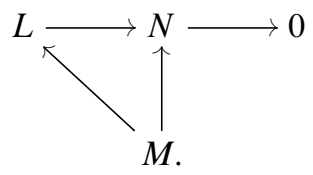

On déduit alors facilement le corollaire suivant :

Corollaire B.2. - Soit $0 \rightarrow M_{0} \rightarrow M_{1} \rightarrow \cdots \rightarrow M_{n} \rightarrow 0$ une suite exacte longue dans $\operatorname{Ban}(A)$. Si $M_{1}, \ldots, M_{n}$ sont projectifs, alors $M_{0}$ est projectif.

B.2.3. Morphismes complètement continus. - Soit $M, N \in \operatorname{Ban}(A)$. Soit $\operatorname{Hom}_{A}(M, N)$ le $A$-module des endomorphismes continus. C'est naturellement un $A$-module de Banach pour la convergence uniforme. Munissons par exemple $M$ et $N$ de deux normes $|\cdot|_{M}$ et $|\cdot|_{N}$. Alors la topologie sur $\operatorname{Hom}_{A}(M, N)$ est celle induite par la norme $|f|=\sup _{m \in M \backslash\{0\}} \frac{|f(m)|_{N}}{|m|_{M}}$. On dit qu'un élément $f \in \operatorname{Hom}_{A}(M, N)$ est de rang fini si $\operatorname{Im}(f)$ est un sous $A$-module de type fini de $N$. On dit qu'un élément $f \in \operatorname{Hom}_{A}(M, N)$ est complètement continu s'il est dans l'adhérence des opérateurs de rang fini.

Supposons que $M=c_{A}(I)$ et $N=c_{A}(J)$ sont deux modules orthonormalisables. Soit $f \in \operatorname{Hom}_{A}(M, N)$. On note $\operatorname{Mat}(f)=\left(a_{i, j}\right)_{(i, j) \in I \times J}$ la matrice de $f$ dans les bases topologiques canoniques $\left(e_{i}\right)_{i \in I}$ et $\left(e_{j}^{\prime}\right)_{j \in J}$ de $c_{A}(I)$ et $c_{A}(J)$. Concrètement, on a $f\left(e_{i}\right)=\sum_{j \in J} a_{j, i} e_{j}^{\prime}$. La proposition suivante se démontre exactement comme [7], prop. 2.4 :

Proposition B.3. - L'opérateur $f$ est complètement continu si et seulement si les colonnes de $\operatorname{Mat}(f)$ tendent uniformément vers $0: \lim _{j \rightarrow \infty} \sup _{i \in I}\left|a_{i, j}\right|=0$.

$4^{\text {e }}$ SÉRIE - TOME $51-2018-$ No $^{\circ}$ 
B.2.4. Déterminant de Fredholm des endomorphismes complètement continus. - Soit $M \in \operatorname{Ban}(A)$ et $f$ un endomorphisme complètement continu. Supposons que $M$ est orthonormalisable et choisissons un isomorphisme $c_{A}(I) \simeq M$. Soit Mat $(f)=\left(a_{i, j}\right)$ la matrice de $f$ dans cette base. Relativement à cette base, on peut calculer le déterminant de Fredholm (on dit aussi série caractéristique) :

$$
\operatorname{det}(1-X f \mid M)=\lim _{J \subset I \text { fini }} \operatorname{det}\left(1-X\left(a_{i, j}\right)_{(i, j) \in J \times J}\right) .
$$

On vérifie exactement comme dans [7], coro. 2.6, que cette définition est indépendante de la base choisie. On voit également que $\operatorname{det}(1-X f)$ est une série de Fredholm.

On peut définir la série caractéristique de $f$ en supposant seulement que $M$ est projectif comme expliqué page 19 de [7] : Soit $N=M \oplus Q$ un module orthonormalisable. On prolonge $f$ en un endomorphisme $\tilde{f}=f \oplus 0$ de $N$. La série caractéristique de $f$ est alors la série caractéristique de $\tilde{f}$.

B.2.5. Théorie de Riesz. - Soit $M$ un module de Banach projectif et $f$ un endomorphisme compact. On note $F(X)$ son déterminant de Fredholm. Supposons qu'on a une factorisation $F(X)=Q(X) S(X)$ de la série de Fredholm en produit de deux séries de Fredholm premières entre elles. On suppose de plus que $Q$ est un polynôme de degré $d$, de coefficient dominant inversible. On note $Q^{\star}(X)=X^{d} Q\left(X^{-1}\right)$ le polynôme réciproque.

ThÉORÈme B.2. - Il existe une unique décomposition $M=M(Q) \oplus N(Q)$ où $M(Q)$ est un A-module projectif de rang d et $Q^{\star}(f)$ est nul sur $M(Q)$ et inversible $\operatorname{sur} N(Q)$.

La première partie du théorème résulte du théorème A4.3 de [10]. Pour vérifier la nullité de $Q^{\star}(f)$ sur $M(Q)$ on raisonne comme dans la démonstration de [7], Thm. 3.3.

\section{B.3. Construction de variétés de Hecke}

Dans cette section nous allons rappeler brièvement comment on construit des variétés de Hecke (voir [10], sect. A et [7], part I, const. 5.7). Soit $M$ un $A$-module de Banach projectif, $H$ une algèbre commutative d'endomorphismes continus $A$-linéaires de $A$ et $U \in H$ un opérateur compact.

Soit $F$ le déterminant de Fredholm de $U$ agissant sur $M$. Soit $Z=V(F) \subset \mathbb{A}_{\operatorname{Spa}\left(A, A^{+}\right)}^{1}$ la variété spectrale. D'après le corollaire B.1, pour tout point $x \in Z$ on possède un voisinage $U$ fini et plat sur son image $w(U)$ dans $\operatorname{Spa}\left(A, A^{+}\right)$. Au-dessus de $w(U)$ on a une factorisation $F=Q S$ où $Q$ est un polynôme et $S$ une série de Fredholm première à $Q$ et $U=V(Q) \subset Z_{\left.\right|_{w(U)}}$. D'après le théorème B.2 on a une décomposition $M=M(Q) \oplus N(Q)$ où $M(Q)$ est l'espace caractéristique associé à $Q$. Il possède naturellement une structure de $\mathscr{O}_{Z}(U)$-module. On définit un faisceau cohérent $\mathscr{M}$ sur $Z$ par $\mathscr{M}(U)=M(Q)$. On note $\mathscr{O}_{E}$ la $\mathscr{O}_{\mathrm{Z}}$-algèbre cohérente engendrée par $H$ dans $\operatorname{End}_{\mathrm{Z}}(\mathscr{M})$. On note $E \rightarrow Z$ l'espace adique associé à $\mathscr{O}_{E}$. C'est la variété de Hecke associée à $(M, H, U)$. La proposition suivante résume la construction et les propriétés de $E$ et $Z$.

Proposition B.4. - Le morphisme $Z \rightarrow \operatorname{Spa}\left(A, A^{+}\right)$est localement quasi fini, plat et partiellement propre. En particulier, localement sur $Z$ et $\mathrm{Spa}\left(A, A^{+}\right)$, il est fini et plat. On possède un faisceau cohérent d'espaces propres généralisés $\mathscr{M}$ sur $\mathscr{O}_{\mathrm{Z}}$ et $\mathscr{O}_{\mathrm{E}}$ agit fidèlement sur $\mathscr{M}$. Le morphisme $E \rightarrow Z$ est fini, le morphisme $E \rightarrow \operatorname{Spa}\left(A, A^{+}\right)$est sans torsion. 


\section{BIBLIOGRAPHIE}

[1] A. Abbès, A. Mokrane, Sous-groupes canoniques et cycles évanescents $p$-adiques pour les variétés abéliennes, Publ. Math. IHÉS 99 (2004), 117-162.

[2] F. Andreatta, C. Gasbarri, The canonical subgroup for families of abelian varieties, Compos. Math. 143 (2007), 566-602.

[3] F. Andreatta, A. Iovita, G. Stevens, Overconvergent modular sheaves and modular forms for $\mathbf{G L}_{2 / F}$, Israel J. Math. 201 (2014), 299-359.

[4] J. Bergdall, R. Pollack, Arithmetic properties of Fredholm series for $p$-adic modular forms, Proc. Lond. Math. Soc. 113 (2016), 419-444.

[5] L. Berger, P. Colmez, Familles de représentations de de Rham et monodromie $p$ adique, Astérisque 319 (2008), 303-337.

[6] P. Berthelot, Cohomologie rigide et cohomologie rigide à supports propres, première partie, prépublication 96-03 https://perso.univ-rennes1.fr/ pierre.berthelot/publis/Cohomologie_Rigide_I.pdf, 1996.

[7] K. Buzzard, Eigenvarieties, in Proceedings of the LMS Durham conference on L-functions and Galois representations, 2004.

[8] K. Buzzard, L. J. P. Kilford, The 2-adic eigencurve at the boundary of weight space, Compos. Math. 141 (2005), 605-619.

[9] R. F. Coleman, Classical and overconvergent modular forms, Invent. math. 124 (1996), 215-241.

[10] R. F. Coleman, $p$-adic Banach spaces and families of modular forms, Invent. math. 127 (1997), 417-479.

[11] R. F. Coleman, The spectral halo, notes, 2014.

[12] R. F. Coleman, B. Mazur, The eigencurve, in Galois representations in arithmetic algebraic geometry (Durham, 1996), London Math. Soc. Lecture Note Ser. 254, Cambridge Univ. Press, Cambridge, 1998, 1-113.

[13] P. Deligne, M. Rapoport, Les schémas de modules de courbes elliptiques, in Modular functions of one variable, II (Proc. Internat. Summer School, Univ. Antwerp, Antwerp, 1972), Lecture Notes in Math. 349, Springer, Berlin, 1973, 143-316.

[14] L. FARgues, La filtration de Harder-Narasimhan des schémas en groupes finis et plats, J. reine angew. Math. 645 (2010), 1-39.

[15] L. Fargues, La filtration canonique des points de torsion des groupes $p$-divisibles, Ann. Sci. Éc. Norm. Supér. 44 (2011), 905-961.

[16] A. Grothendieck, Éléments de géométrie algébrique. IV. Étude locale des schémas et des morphismes de schémas. II, Publ. Math. IHÉS 24 (1965), 231.

[17] U. Hartl, R. Pink, Vector bundles with a Frobenius structure on the punctured unit disc, Compos. Math. 140 (2004), 689-716.

[18] R. Huber, Continuous valuations, Math. Z. 212 (1993), 455-477.

[19] R. Huber, A generalization of formal schemes and rigid analytic varieties, Math. $Z$. 217 (1994), 513-551.

[20] R. Huber, étale cohomology of rigid analytic varieties and adic spaces, Aspects of Mathematics, E30, Friedr. Vieweg \& Sohn, Braunschweig, 1996.

$4^{\mathrm{e}}$ SÉRIE - TOME $51-2018$ - No 3 
[21] N. M. Katz, $p$-adic properties of modular schemes and modular forms, in Modular functions of one variable, III (Proc. Internat. Summer School, Univ. Antwerp, Antwerp, 1972), Lecture Notes in Math. 350, Springer, 1973, 69-190.

[22] R. Liu, D. Wan, L. XiaO, The eigencurve over the boundary of weight space, Duke Math. J. 166 (2017), 1739-1787.

[23] W. LütкевонмеRт, Der Satz von Remmert-Stein in der nichtarchimedischen Funktionentheorie, Math. Z. 139 (1974), 69-84.

[24] W. Messing, The crystals associated to Barsotti-Tate groups : with applications to abelian schemes, Lecture Notes in Math. 264, Springer, Berlin-New York, 1972.

[25] V. Pilloni, Overconvergent modular forms, Ann. Inst. Fourier (Grenoble) 63 (2013), 219-239.

[26] P. Scholze, On torsion in the cohomology of locally symmetric varieties, Ann. of Math. 182 (2015), 945-1066.

[27] J-P. SERre, Endomorphismes complètement continus des espaces de Banach p-adiques, Publ. Math. IHÉS 12 (1962), 69-85.

[28] J. T. Tate, p-divisible groups, in Proc. Conf. Local Fields (Driebergen, 1966), Springer, Berlin, 1967, 158-183.

[29] Y. Tian, Canonical subgroups of Barsotti-Tate groups, Ann. of Math. 172 (2010), 955988.

[30] P. Valabrega, A few theorems on completion of excellent rings, Nagoya Math. J. 61 (1976), 127-133.

[31] A. WiLes, On ordinary $\lambda$-adic representations associated to modular forms, Invent. math. 94 (1988), 529-573.

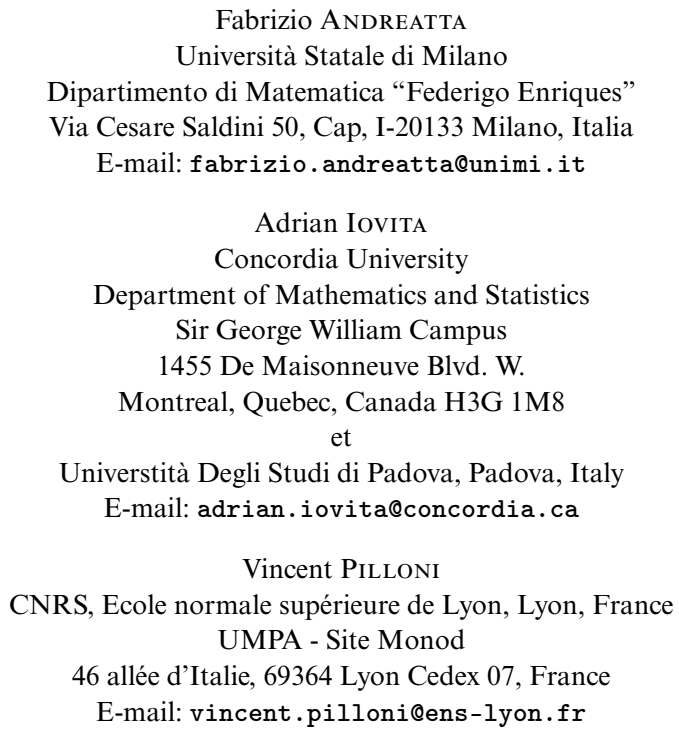


